\title{
Ranking and Repulsing Supermartingales for Reachability in Probabilistic Programs
}

\author{
Toru Takisaka $^{1}$, Yuichiro Oyabu ${ }^{1,2}$, Natsuki Urabe ${ }^{3}$, and Ichiro Hasuo ${ }^{1,2}$ \\ 1 National Institute of Informatics, Japan \\ 2 The Graduate University for Advanced Studies (SOKENDAI), Japan \\ ${ }^{3}$ University of Tokyo, Japan
}

\begin{abstract}
Computing reachability probabilities is a fundamental problem in the analysis of probabilistic programs. This paper aims at a comprehensive and comparative account of various martingale-based methods for over- and underapproximating reachability probabilities. Based on the existing works that stretch across different communities (formal verification, control theory, etc.), we offer a unifying account. In particular, we emphasize the role of order-theoretic fixed points - a classic topic in computer science - in the analysis of probabilistic programs. This leads us to two new martingale-based techniques, too. We also make an experimental comparison using our implementation of template-based synthesis algorithms for those martingales.
\end{abstract}

\section{Introduction}

Computing reachability probabilities is a fundamental problem in the analysis of probabilistic systems. It is known that probabilistic model checking titleems can be solved via reachability probabilities [4], much like nondeterministic model checking problems are reduced to emptiness and hence to reachability [34]. While the computation of reachability probabilities for finite-state systems is effectively solved by linear programming, the problem becomes much more challenging for probabilistic programs-a paradigm that programs. The line 4 means that attracts growing attention as a programming language the value of $z$ is randomly sampled foundation for machine learning [17]—because their from the interval $[-2,1]$. transition graphs are infinite in general.

Reachability probabilities of probabilistic programs with while loops are clearly not computable, because the problem encompasses termination of (non-probabilistic) while programs. Therefore the existing research efforts have focused on sound approximation methods for reachability probabilities. An approach that is widely used in the literature is to use ranking supermartingales - a probabilistic analogue of ranking functions-as a witness for the qualitative question of almost-sure reachability. Ranking supermartingales are amenable to template-based synthesis [8|11]9], making them appealing from the automatic analysis point of view. Recently, methods for quantitatively underapproximating reachability probabilities are also proposed in [13]33]. 
Table 1. Martingale-based techniques for approximation of reachability probabilities. MC stands for Markov chains, and PP stands for probabilistic programs

\begin{tabular}{|c|c|c|c|}
\hline & & certificate for & from \\
\hline \multirow{2}{*}{$\begin{array}{c}\text { ranking } \\
\text { (super- and sub-) } \\
\text { martingale for } \\
\text { under-approximation }\end{array}$} & $\begin{array}{c}\text { additive } \\
\text { supermartingale } \\
\text { (ARnkSupM, \$5) }\end{array}$ & $\mathbb{E}($ steps to $C) \leq$ ? & 248 \\
\hline & $\begin{array}{c}\gamma \text {-scaled } \\
\text { submartingale } \\
(\gamma \text {-SclSubM, } 6)\end{array}$ & $\mathbb{P}($ reach $C) \geq$ ? & $\begin{array}{c}\text { this paper for PP, } \\
\text { following categorical } \\
\text { observations in [33] for } \mathrm{MC}\end{array}$ \\
\hline \multirow{2}{*}{$\begin{array}{c}\text { repulsing } \\
\text { supermartingale for } \\
\text { over-approximation }\end{array}$} & $\begin{array}{c}\text {-decreasing } \\
\text { supermartingale } \\
(\varepsilon-\operatorname{RepSupM}, \sqrt{3})\end{array}$ & $\begin{array}{c}\mathbb{P}(\text { reach } C) \leq ? \\
\mathbb{P}(\text { reach } C)<?\end{array}$ & $\begin{array}{c}{[13], \text { derived from }} \\
\text { Azuma's martingale } \\
\text { concentration inequality }\end{array}$ \\
\hline & $\begin{array}{c}\text { nonnegative } \\
\text { supermartingale } \\
\text { (NNRepSupM, } 4 \text {. }\end{array}$ & $\mathbb{P}($ reach $C) \leq ?$ & $\begin{array}{c}\text { this paper, derived from } \\
\text { the Knaster-Tarski theorem } \\
\text { ([26]31], without nondeterminism, } \\
\text { derived from Markov's } \\
\text { concentration inequality) }\end{array}$ \\
\hline
\end{tabular}

The dual question of overapproximating reachability probabilities, which can then be used to qualitatively refute almost-sure reachability, is also considered. In the control theory, supermartingales are used as a probabilistic counterpart of barrier certificates [26]31]. A similar idea is recently used for the purpose of synthesizing stochastic invariants for probabilistic programs [13]. Here an overapproximation of reachability probability serves as quantitative verification for safety: it gives an upper bound for the probability that the system or the program reaches a bad state.

Table 11 lists four supermartingale-based techniques for over- and underapproximating reachability probabilities. The table is not meant to be exhaustive-still, it shows that multiple methods have been introduced and studied, in different communities (formal verification, control theory, etc.) and with different mathematical backgrounds (ranking functions, martingale concentration inequalities, etc.).

The current work aims at a comprehensive and comparative account of those martingalebased techniques in Table 1 Central to our account is the role of order-theoretic fixed points, a classic topic in theoretical computer science. More specifically, we characterize our objectives - namely reachability probability and expected reaching time-as suitable least fixed points. It turns out that a large part of the theory of martingale-based methods can be developed based on this order-theoretic characterization, without using mathematical gadgets unique to probabilistic settings such as martingale concentration inequalities. Our contributions are summarized as follows.

- A comprehensive and comparative account of different martingale-based techniques for approximating reachability probabilities. We identify their key mathematical principles to be order-theoretic fixed points and martingale concentration inequalities, and we emphasize the role of the former.

- We introduce two martingale-based techniques that seem to be new, namely $\gamma$ SclSubM and NNRepSupM in Table 1 Their purely probabilistic versions have been in the literature: $\gamma$-SclSubM is from a category-theoretic account in [33], and 
NNRepSupM is from control theory [31]. We extend them to probabilistic programs that additionally have nondeterminism. Moreover, completeness of ARnkSupM for probabilistic programs with real-valued variables seems to be new.

- We formalize those techniques, taking probabilistic programs (with nondeterminism) as the target of analyses. We investigate soundness and completeness of the techniques in Table 11. While the order-theoretic fixed-point foundation gives us clear theoretical guidance, additional nondeterminism requires us to carefully establish measure-theoretic arguments.

- We implemented template-based automated synthesis algorithms for $\gamma$-SclSubM, $\varepsilon$-RepSupM and NNRepSupM, following [8]11]. Our experimental results suggest the advantage of $\gamma$-SclSubM in quantitative reasoning, and the comparative advantage of NNRepSupM over $\varepsilon$-RepSupM in the quality of bounds.

The paper is organized as follows. Preliminaries are in $\$ 2$, where we introduce our system models (pCFGs) for operational semantics of probabilistic programs, and review the theory of order-theoretic fixed points (the Knaster-Tarski and Cousot-Cousot theorems). In 3 - 6 we discuss the four techniques in Table 1 offering a unifying account based on order-theoretic fixed points, and providing some new techniques and results. In $\$ 7$ we give implementations and experiment results of template-based synthesis. After discussion of related work in $\$ 8$, we conclude in $\$ 9$.

\section{Preliminaries}

We first fix some notations. We write $\mathbb{N}$ and $\mathbb{R}$ for the set of all natural numbers (i.e. nonnegative integers) and reals, respectively. We use subscripts to denote subsets of $\mathbb{N}$ and $\mathbb{R}$; for example, $\mathbb{R}_{\geq 0}$ denotes the set of all nonnegative reals. We write $X^{*}, X^{+}, X^{\omega}$ for the sets of all finite, nonempty finite, and infinite sequences of elements of $X$, respectively.

We use the Borel measurable structure $\mathcal{B}(\mathbb{R})$ of the set $\mathbb{R}$ of real numbers. This induces the measurable structures of all the other sets used in this paper: $\mathbb{R}^{k}$ where $k \in \mathbb{N}, X \times \mathbb{R}^{k}$ where $X$ is finite, and so on. The induced measurable structures are defined in a standard manner: for example, $X \times \mathbb{R}^{k}$ where $X$ is finite, it is given by $\mathcal{B}\left(X \times \mathbb{R}^{k}\right)=\left(\mathcal{B}\left(\mathbb{R}^{k}\right)\right)^{X}$. The set of probability distributions on $(X, \mathcal{B}(X))$ is denoted by $\mathcal{D}(X)$. The Dirac measure on $x \in X$ is denoted by $\delta_{x}$. The support $\operatorname{supp}(d)$ of $d \in$ $\mathcal{D}(X)$ is defined by $\operatorname{supp}(d)=\{x \in X \mid$ for any $A \in \mathcal{B}(X), x \in A$ implies $d(A)>$ $0\}$. The set of all Borel measurable function from $X$ to $Y$ is denoted by $\mathcal{B}(X, Y)$. The functions $\mathbf{0}$ and $\mathbf{1}$ are the real-valued constant function of which coefficient is 0 and 1 , respectively.

\subsection{Probabilistic Control Flow Graphs (pCFGs)}

We take the notion of pCFG from [2] and use it as our model of probabilistic systems. pCFGs can be thought of as a subclass of Markov decision processes (MDPs), but tailored for operational semantics of probabilistic programs (2.2). 


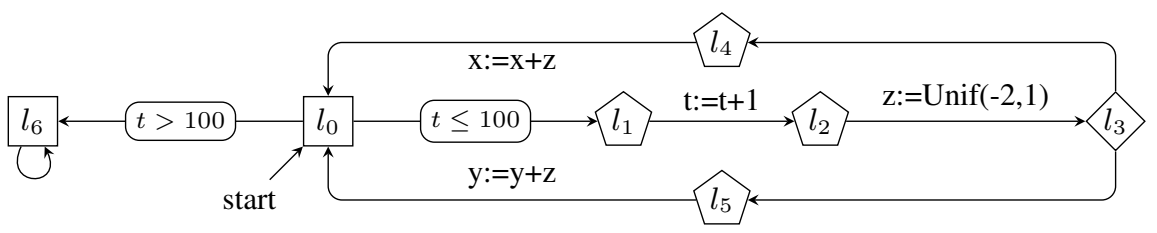

Fig. 2. The pCFG that models the probabilistic program in Fig. 1 Rectangles, diamonds, and pentagons represent deterministic, nondeterministic and assignment locations, respectively. The variables are initially set $x:=2, y:=2$ and $t:=0$.

Definition 2.1 (pCFG, [2]). A probabilistic control flow $\operatorname{graph}(p C F G)$ is a tuple $\Gamma=$ $\left(L, V, l_{\text {init }}, \boldsymbol{x}_{\text {init }}, \mapsto, \mathrm{Up}, \operatorname{Pr}, G\right)$ consisting of the following components.

- A finite set $L$ of locations, equipped with a partition $L=L_{N}+L_{P}+L_{D}+L_{A}$ into nondeterministic, probabilistic, deterministic and assignment locations.

- A finite set $V=\left\{x_{1}, \ldots, x_{|V|}\right\}$ of program variables.

- An initial location $l_{\mathrm{init}} \in L$, and an initial valuation vector $\boldsymbol{x}_{\mathrm{init}} \in \mathbb{R}^{V}$.

- A transition relation $\mapsto \subseteq L \times L$ which is total (each location has a successor). For $l \in L \backslash L_{A}$, we write $\operatorname{succ}(l)$ to denote the set of all successors of $l$, i.e. $\operatorname{succ}(l)=\left\{l^{\prime} \in L \mid l \mapsto l^{\prime}\right\}$. We require that each assignment location $l \in L_{A}$ has a unique successor; in this case, $\operatorname{succ}(l)$ denotes this unique location.

- An update function $\mathrm{Up}: L_{A} \rightarrow V \times \mathcal{U}$, where $\mathcal{U}=\mathcal{B}\left(\mathbb{R}^{V}, \mathbb{R}\right) \cup \mathcal{D}(\mathbb{R}) \cup \mathcal{B}(\mathbb{R})$. Here, three components of $\mathcal{U}$ represent deterministic, probabilistic and nondeterministic assignment, respectively.

- A family $\operatorname{Pr}=\left(\operatorname{Pr}_{l} \in \mathcal{D}(\operatorname{succ}(l))\right)_{l \in L_{P}}$ of probability distributions.

- A guard function $G: L_{D} \times L \rightarrow \mathcal{B}\left(\mathbb{R}^{V}\right)$ such that, for each $l \in L_{D}$, the following hold: (collective exhaustion) $\bigcup_{l \mapsto l^{\prime}} G\left(l, l^{\prime}\right)=\mathbb{R}^{V}$; and (mutual exclusion) $l \mapsto l^{\prime}$, $l \mapsto l^{\prime \prime}$ and $l^{\prime} \neq l^{\prime \prime}$ imply $G\left(l, l^{\prime}\right) \cap G\left(l, l^{\prime \prime}\right)=\emptyset$. We write $\boldsymbol{x} \models G\left(l, l^{\prime}\right)$ if $\boldsymbol{x} \in G\left(l, l^{\prime}\right)$.

A configuration of a pCFG $\Gamma$ is a pair $(l, \boldsymbol{x}) \in L \times \mathbb{R}^{V}$ of a location and a vector. A successor $\left(l^{\prime}, \boldsymbol{x}^{\prime}\right)$ of a configuration $(l, \boldsymbol{x})$ is a one such that $l \mapsto l^{\prime}$ and

- if $l \in L_{N} \cup L_{P}$ then $\boldsymbol{x}^{\prime}=\boldsymbol{x}$;

- if $l \in L_{D}$ then $\boldsymbol{x}^{\prime}=\boldsymbol{x}$ and $\boldsymbol{x} \models G\left(l, l^{\prime}\right)$; and

- if $l \in L_{A}$ and $\operatorname{Up}(l)=\left(x_{j}, u\right)$, then $\boldsymbol{x}^{\prime}=\boldsymbol{x}\left(x_{j} \leftarrow a\right)$. Here $\boldsymbol{x}\left(x_{j} \leftarrow a\right)$ denotes an update of the vector (the $x_{j}$-component of $\boldsymbol{x}$ is replaced by $a$ ), and i) $a=u(\boldsymbol{x})$ if $u \in \mathcal{B}\left(L \times \mathbb{R}^{V}, \mathbb{R}\right)$, ii) $a \in \operatorname{supp}(u)$ if $u \in \mathcal{D}(\mathbb{R})$, and iii) $a \in u$ if $u \in \mathcal{B}(\mathbb{R})$.

A finite path of $\Gamma$ is a finite sequence $c_{0}, c_{1}, \ldots, c_{k}$ of configurations where $c_{i}$ is a successor of $c_{i-1}$ for each $i$. Similarly, A run of $\Gamma$ is an infinite sequence $c_{0}, c_{1}, \ldots$ of configurations such that each $c_{i}$ is a successor of $c_{i-1}$.

Schedulers resolve nondeterminism. Given a history $c_{0} \ldots c_{i}$ of configurations, it gives a distribution of the successor's location or valuation vector. We assume that a scheduler is universally measurable, which is standard in control theory (see e.g. [5]).

If a pCFG $\Gamma$ and a scheduler $\sigma$ for $\Gamma$ are given, then the behavior of $\Gamma$ is determined for each initial configuration $c_{0}$; we represent it by the map $\mu_{-}^{\sigma}:\left(L \times \mathbb{R}^{V}\right)^{+} \rightarrow \mathcal{D}(L \times$ 
$\mathbb{R}^{V}$ ). For each nonempty sequence $c_{0} \ldots c_{i}$ the distribution $\mu_{c_{0} \ldots c_{i}}^{\sigma}$ is, intuitively, the distribution of the next configuration given a current history $c_{0} \ldots c_{i}$ of configurations under the scheduler $\sigma$. For the set $\operatorname{Sch}_{\Gamma}$ of all schedulers for $\Gamma$ we define the following.

Definition 2.2 (reachability probabilities $\mathbb{P}_{C, \sigma}^{\text {reach }}, \overline{\mathbb{P}}_{C}^{\text {reach }}, \mathbb{P}_{C}^{\text {reach }}$ ). Let $\Gamma$ be a pCFG. The reachability probability $\mathbb{P}_{C, \sigma}^{\text {reach }}(c)$ from a configuration $c_{0} \in L \times \mathbb{R}^{V}$ to a region $C \in \mathcal{B}\left(L \times \mathbb{R}^{V}\right)$ under a scheduler $\sigma \in \operatorname{Sch}_{\Gamma}$ is defined by

$$
\mathbb{P}_{C, \sigma}^{\text {reach }}\left(c_{0}\right)=\sum_{i \geq 1} \int_{L \times \mathbb{R}^{V} \backslash C} \mu_{c_{0}}^{\sigma}\left(\mathrm{d} c_{1}\right) \ldots \int_{L \times \mathbb{R}^{V} \backslash C} \mu_{c_{0} \ldots c_{i-2}}^{\sigma}\left(\mathrm{d} c_{i-1}\right) \int_{C} \mathbf{1} \mu_{c_{0} \ldots c_{i-1}}^{\sigma}\left(\mathrm{d} c_{i}\right)
$$

for the case of $c_{0} \notin C$, and $\mathbb{P}_{C, \sigma}^{\mathrm{reach}}\left(c_{0}\right)=1$ otherwise. The upper reachability probability $\overline{\mathbb{P}}_{C}^{\text {reach }}(c)$ from $c$ to $C$ is defined by $\overline{\mathbb{P}}_{C}^{\text {reach }}(c)=\sup _{\sigma \in \operatorname{Sch}_{\Gamma}} \mathbb{P}_{C, \sigma}^{\text {reach }}(c)$; the lower reachability probability $\underline{\mathbb{P}}_{C}^{\mathrm{reach}}(c)$ is defined by $\underline{\mathbb{P}}_{C}^{\mathrm{reach}}(c)=\inf _{\sigma \in \operatorname{Sch}_{\Gamma}} \mathbb{P}_{C, \sigma}^{\mathrm{reach}}(c)$.

Definition 2.3 (reaching times $\mathbb{E}_{C, \sigma}^{\text {steps }}, \overline{\mathbb{E}}_{C}^{\text {steps }}, \underline{\mathbb{E}}_{C}^{\text {steps }}$ ). Let $\Gamma$ be a pCFG. The expected reaching time of $\Gamma$ from a configuration $c_{0} \in L \times \mathbb{R}^{V}$ to $C \in \mathcal{B}\left(L \times \mathbb{R}^{V}\right)$ under a scheduler $\sigma \in \operatorname{Sch}_{\Gamma}$ is defined by $\mathbb{E}_{C, \sigma}^{\text {steps }}\left(c_{0}\right)=0$ for $c_{0} \in C$; for $c_{0} \notin C$ it is defined by

$\mathbb{E}_{C, \sigma}^{\text {steps }}\left(c_{0}\right)=\sum_{i \geq 1} i \cdot \int_{L \times \mathbb{R}^{V} \backslash C} \mu_{c_{0}}^{\sigma}\left(\mathrm{d} c_{1}\right) \ldots \int_{L \times \mathbb{R}^{V} \backslash C} \mu_{c_{0} \ldots c_{i-2}}^{\sigma}\left(\mathrm{d} c_{i-1}\right) \int_{C} \mathbf{1} \mu_{c_{0} \ldots c_{i-1}}^{\sigma}\left(\mathrm{d} c_{i}\right)$

if $\mathbb{P}_{C, \sigma}^{\text {reach }}\left(c_{0}\right)=1$, or $\mathbb{E}_{C, \sigma}^{\text {steps }}\left(c_{0}\right)=\infty$ otherwise. The upper expected reaching time $\overline{\mathbb{E}}_{C}^{\text {steps }}(c)$ of $\Gamma$ from $c$ to $C$ is $\overline{\mathbb{E}}_{C}^{\text {steps }}(c)=\sup _{\sigma \in \mathrm{Sch}_{\Gamma}} \mathbb{E}_{C, \sigma}^{\text {steps }}(c)$, and the lower expected reaching time is $\underline{\mathbb{E}}_{C}^{\text {steps }}(c)=\inf _{\sigma \in \operatorname{Sch}_{\Gamma}} \mathbb{E}_{C, \sigma}^{\text {steps }}(c)$.

\subsection{Probabilistic Programs: APP and PPP}

The goal of this paper is the reachability analysis of imperative programs with probabilistic and nondeterministic branching. We consider two languages taken from [13]11], called affine probabilistic programs (APP) and polynomial probabilistic programs (PPP). The two languages differ only in the arithmetic expressions allowed in the assignment commands and Boolean expressions. For example, the assignment command $x:=$ $x y+x+1$ is allowed in PPP but not in APP; $x:=3 x+2 y-1$ is allowed in both since its right-hand side is an affine expression.

Both APP and PPP have the standard control structure in imperative languagessuch as if-branches and while-loops. APP and PPP additionally have nondeterministic and probabilistic if-branches (if $\star$ then $\ldots$ and if $\operatorname{prob}(p)$ then ..., respectively, where $p \in[0,1])$. They also have nondeterministic and probabilistic assignment commands: $x:=\operatorname{ndet} A$ where a value is chosen from a set $A \subseteq \mathbb{R}$; and $x:=d$ where a value is sampled from a probability distribution $d$ over $\mathbb{R}$.

The definition of the semantical model pCFG $(2.1)$ mirrors the structure of these languages. The translation from APP/PPP to $\mathrm{pCFGs}$ is straightforward and omitted. 


\subsection{Order-Theoretic Foundation of Fixed Points}

Order-theoretic fixed points are central to computer science, for recursive computation, inductive/coinductive datatypes and reasoning and specification of reactive behaviors, etc. In general, a fixed-point equation can have multiple solutions; often we are interested in extremal solutions: least fixed points (lfp's, for liveness, induction, etc.) and greatest ones (gfp's, for safety, coinduction, etc.). The following fundamental results (in a simple setting of complete lattices) give two different characterizations of lfp's and gfp's.

Theorem 2.4. Let $(L, \sqsubseteq)$ be a complete lattice, and $f: L \rightarrow L$ be a monotone function. Then $f$ has the least fixed point $\mu f$ and the greatest $\nu f$. Moreover,

1. (Knaster-Tarski) The lfp is the least pre-fixed point: $\mu f=\min \{l \in L \mid f(l) \sqsubseteq l\}$. Similarly, the gfp is the greatest post-fixed point: $\nu f=\max \{l \in L \mid l \sqsubseteq f(l)\}$.

2. (Cousot-Cousot [14]) The (potentially transfinite) ascending chain $\perp \sqsubseteq f(\perp) \sqsubseteq$ $f^{2}(\perp) \sqsubseteq \cdots$ stabilizes to $\mu \mathrm{f}$. Here $f^{\alpha}(\perp)$ is defined by obvious induction: $f^{\alpha+1}(\perp)=$ $f\left(f^{\alpha}(\perp)\right)$ for a successor ordinal; and $f^{\alpha}(\perp)=\bigsqcup_{\beta<\alpha} f^{\beta}(\perp)$ for a limit ordinal. Similarly, the descending chain $\top \sqsupseteq f(\top) \sqsupseteq \cdots$ stabilizes to $\nu f$.

From these characterizations we can derive the following reasoning principles.

Corollary 2.5. $\quad-(\boldsymbol{l f p}$-KT) $f(l) \sqsubseteq l$ implies $\mu f \sqsubseteq l$.

- $(\boldsymbol{g} f \boldsymbol{p}-\boldsymbol{K} \boldsymbol{T}) l \sqsubseteq f(l)$ implies $l \sqsubseteq \nu f$.

- (lfp-CC) For each ordinal $\alpha, f^{\alpha}(\perp) \sqsubseteq \mu f$.

- (gfp-CC) For each ordinal $\alpha, \nu f \sqsubseteq f^{\alpha}(\top)$.

The arguments so far are symmetric for lfp's and gfp's. However, if one turns to the common proof methods for lfp specifications (termination, reachability, liveness) and those for gfp specifications (safety), a strong contrast emerges. Here is an example.

Lemma 2.6. Let $(S, \mapsto \subseteq S \times S)$ be a Kripke frame, $s_{0} \in S$ and $C \subseteq S$.

- (Invariant for safety) Let $I \subseteq S$ be an invariant, that is, $I \subseteq \square I$. Here $\square I$ is defined by $\square I=\left\{s \in S \mid s \mapsto s^{\prime}\right.$ implies $\left.s^{\prime} \in I\right\}$. Assume also that $I \cap C=\emptyset$. Then $s_{0} \in I$ implies that there is no path from $s_{0}$ to $C$.

- (Ranking function for liveness) Let $\eta: S \rightarrow \mathbb{N} \cup\{\infty\}$ be a ranking function for $C$. That is, 1) for each $s \in S \backslash C$, there is a successor $s^{\prime}$ such that $\eta(s) \geq \eta\left(s^{\prime}\right)+1$; and 2) for each $s \in S, \eta(s)=0$ implies $s \in C$. Then, $\eta\left(s_{0}\right) \neq \infty$ implies that there is a path from $s_{0}$ to $C$.

The difference between the two methods is accounted for by the fact that, in Cor.2.5, two items give under-approximations while the other two give over-approximations. It is clear that the in|Knaster-Tarski|Cousot-Cousot \begin{tabular}{c||c|c}
\hline \hline lfp & overapprox. & underapprox. \\
\hline gfp & underapprox. & overapprox.
\end{tabular} variant method in Lem. 2.6 comes from (gfp-KT) of Cor. 2.5. Its dual, (lfp-KT), gives only an overapproximation $l$ - it can be used for refutation but not for verification. Similarly, ranking functions come from (lfp-CC) - the role of well-foundedness of the value domain $\mathbb{N}$ mirrors the structure of ordinals. Its dual (gfp-CC) only gives an overapproximation of $\nu f$. The situation is summarized in the above table. 
The above foundations underpin our technical developments: this is because reachability probabilities and reaching times are characterized as least fixed points. We note that our semantical domains $L$ in later sections need not be complete lattices. In those cases we exploit the $\omega$ - and $\omega^{\mathrm{op}}$-cpo structures, the corresponding continuity of $f$, and the Kleene theorem. The last is understood as a variation of the Cousot-Cousot theorem.

\subsection{Invariants and the Nexttime Operations}

In $₫ 3$-6 the following definitions will be used.

Definition 2.7 ((pure) invariant for pCFG). Let $\Gamma$ be a pCFG. A measurable set $I \in$ $\mathcal{B}\left(L \times \mathbb{R}^{V}\right)$ is called a (pure) invariant for $\Gamma$ if $\left(l_{\text {init }}, \boldsymbol{x}_{\text {init }}\right) \in I$, and for each $(l, \boldsymbol{x}) \in I$, if $\left(l^{\prime}, \boldsymbol{x}^{\prime}\right)$ is a successor of $(l, \boldsymbol{x})$ then $\left(l^{\prime}, \boldsymbol{x}^{\prime}\right) \in I$.

Definition 2.8 (the "nexttime" operation $\overline{\mathbb{X}}, \underline{\mathbb{X}}$ ). Let $\Gamma$ be a pCFG, $I$ be a pure invariant and $\mathbb{K} \in \mathcal{B}(\mathbb{R})$. For a measurable $\eta: I \rightarrow \mathbb{K}$ we define the function $\overline{\mathbb{X}} \eta$ of the same type as $\eta$ as follows, provided the right-hand side of each equation is well-defined.

- For $l \in L_{N},(\overline{\mathbb{X}} \eta)(l, \boldsymbol{x})=\max _{l \mapsto l^{\prime}} \eta\left(l^{\prime}, \boldsymbol{x}\right)$.

- For $l \in L_{P},(\overline{\mathbb{X}} \eta)(l, \boldsymbol{x})=\sum_{l \mapsto l^{\prime}} \operatorname{Pr}_{l}\left(l^{\prime}\right) \eta\left(l^{\prime}, \boldsymbol{x}\right)$.

- For $l \in L_{D},(\overline{\mathbb{X}} \eta)(l, \boldsymbol{x})=\eta\left(l^{\prime}, \boldsymbol{x}\right)$ where $l^{\prime}$ is the unique location s.t. $\boldsymbol{x} \models G\left(l, l^{\prime}\right)$.

- For $l \in L_{A}$, let $\operatorname{Up}(l)=\left(x_{j}, u\right)$.

- $(\overline{\mathbb{X}} \eta)(l, \boldsymbol{x})=\eta(\operatorname{succ}(l), u(\boldsymbol{x}))$ if $u$ is a measurable function.

- $(\overline{\mathbb{X}} \eta)(l, \boldsymbol{x})=\int_{x \in \operatorname{supp}(u)} \eta\left(\operatorname{succ}(l), \boldsymbol{x}\left(x_{j} \leftarrow x\right)\right) \mathrm{d} u$ if $u$ is a distribution.

- $(\overline{\mathbb{X}} \eta)(l, \boldsymbol{x})=\sup _{x \in u} \eta\left(\operatorname{succ}(l), \boldsymbol{x}\left(x_{j} \leftarrow x\right)\right)$ if $u$ is a measurable set.

The function $\underline{\mathbb{X}} \eta: I \rightarrow \mathbb{K}$ is defined as above, but replacing max with min in the first line and sup with inf in the last.

Proposition 2.9. We define a pointwise partial order $\sqsubseteq$ on $\mathcal{B}(I, \mathbb{K})$, i.e. $f \sqsubseteq g$ if and only if $f(c) \leq g(c)$ holds for every $c \in I$. Let $\mathbb{K}$ be a proper closed convex subset of $\mathbb{R} \cup\{ \pm \infty\}$. Then $\overline{\mathbb{X}} \eta$ and $\underline{\mathbb{X}} \eta$ are well-defined for every $\eta \in \mathcal{B}(I, \mathbb{K})$, and the following hold.

1. The operators $\overline{\mathbb{X}}$ and $\underline{\mathbb{X}}$ are monotone endofunctions over $\mathcal{B}(I, \mathbb{K})$. In particular, $\overline{\mathbb{X}} \eta$ and $\underline{\mathbb{X}} \eta$ are Borel measurable for any $\eta \in \mathcal{B}(I, \mathbb{K})$.

2. $\overline{\mathbb{X}}$ is $\omega$-continuous, and $\mathbb{X}$ is $\omega^{\mathrm{op}}$-continuous.

\section{$3 \varepsilon$-Decreasing Repulsing Supermartingales ( $\varepsilon$-RepSupM)}

In $\sqrt[336]{6}$ we will discuss the four martingale-based techniques in Table 1 . Here we briefly review the notion of $\varepsilon$-decreasing repulsing supermartingale ( $\varepsilon$-RepSupM) from [13]. It is, to the best of our knowledge, the only existing martingale-based notion for overapproximating reachability probabilities.

Definition 3.1 ( $\varepsilon$-RepSupM [13]). Let $\Gamma$ be a pCFG, $I$ be a pure invariant, and $C \subseteq I$ be a Borel set. An $\varepsilon$-repulsing supermartingale ( $\varepsilon-\operatorname{RepSupM})$ for $C$ supported by $I$ is a measurable function $\eta: I \rightarrow \mathbb{R}$ such that i) $\eta(c) \geq(\overline{\mathbb{X}} \eta)(c)+\varepsilon$ for each $c \in I \backslash C$, and ii) $\eta(c) \geq 0$ for each $c \in C$. 
Theorem 3.2 (soundness, [13]). Suppose there exists an $\varepsilon$-RepSupM for $C$ supported by I such that $\eta\left(l_{\text {init }}, \boldsymbol{x}_{\text {init }}\right)<0$. Further assume that $\eta$ has $\kappa$-bounded differences for some $\kappa>0$, i.e. for each $c \in I$ and its successor $c^{\prime}$ it holds $\left|\eta(c)-\eta\left(c^{\prime}\right)\right| \leq \kappa$. Let $\gamma=e^{-\frac{\varepsilon^{2}}{2(\kappa+\varepsilon)^{2}}}$ and $\alpha=e^{\frac{\varepsilon \cdot \eta\left(l_{\text {init }}, \boldsymbol{x}_{\text {init }}\right)}{(\kappa+\varepsilon)^{2}}}$.

1. We have the following inequality:

$$
\overline{\mathbb{P}}_{C}^{\text {reach }}\left(l_{\text {init }}, \boldsymbol{x}_{\text {init }}\right) \leq \alpha \cdot \frac{\gamma^{\left\lceil\left|\eta\left(l_{\text {init }}, \boldsymbol{x}_{\text {init }}\right)\right| / \kappa\right\rceil}}{1-\gamma} .
$$

2. If the right-hand side of (1) is greater than 1 , still $\overline{\mathbb{P}}_{C}^{\text {reach }}\left(l_{\text {init }}, \boldsymbol{x}_{\text {init }}\right)<1$ holds.

We note that for any $\eta \in \mathcal{B}(I, \mathbb{R})$ that has $\kappa$-bounded differences, the function $\overline{\mathbb{X}} \eta$ is well-defined. The bound in (1) is derived from Azuma's concentration inequality, a well-known martingale concentration lemma that exploits $\kappa$-bounded differences. $\varepsilon$ RepSupM is not complete: there exist a pCFG $\Gamma$ and a set $C$ of configurations such that $\overline{\mathbb{P}}_{C}^{\text {reach }}<1$ but no $\varepsilon$-RepSupM can prove it. See Fig. 3 below.

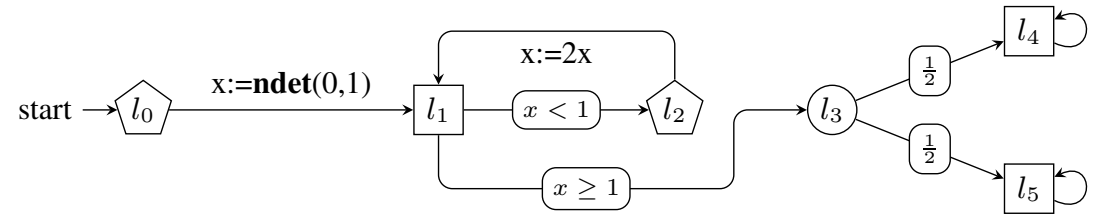

Fig. 3. An example of incompleteness of $\varepsilon$-RepSupM. Probabilistic locations are depicted by circles. This pCFG satisfies $\overline{\mathbb{P}}_{\left\{l_{5}\right\} \times \mathbb{R}}^{\text {reach }}\left(l_{0}, 0\right)=\frac{1}{2}$ but no $\varepsilon$-RepSupM can refute its a.s. reachability. Indeed, any $\varepsilon$-RepSupM $\eta$ for $\left\{l_{5}\right\} \times \mathbb{R}$ must satisfy $\lim _{x \rightarrow+0} \eta\left(l_{1}, x\right)=\infty$ due to the $\varepsilon$ decreasing condition, but such an $\eta$ cannot have $\kappa$-bounded differences at $\left(l_{0}, 0\right)$.

\section{Nonnegative Repulsing Supermartingales (NNRepSupM)}

We move on to another notion for overapproximating reachability probabilities, nonnegative repulsing supermartingale (NNRepSupM). We believe this is new. Compared to the notion of $\varepsilon$-RepSupM, NNRepSupM has the following features.

- NNRepSupM is derived from the theory of order-theoretic fixed points (2.3), unlike $\varepsilon$-RepSupM that relies on Azuma's martingale concentration lemma.

- Consequently, we can show soundness and completeness of NNRepSupM rather easily, while $\varepsilon$-RepSupM is sound but not complete.

- We experimentally observe that NNRepSupM often gives better bounds (77).

The definition of NNRepSupM resembles probabilistic barrier certificates used in control theory [26|31]. Our technical contributions are the following: i) we develop the theory of NNRepSupM in the presence of nondeterminism, while the settings in [26]31] are purely probabilistic; and ii) we characterize NNRepSupM in the general terms of order-theoretic fixed points ( 2.3$)$, unlike the previous theory in [26]31] that relies on 
Markov's martingale concentration lemma 4 The latter unveils the mathematical similarity between NNRepSupM and ARnkSupM ( $(55)$.

The notion comes with upper and lower variants. They are used to overapproximate $\overline{\mathbb{P}}_{C}^{\text {reach }}$ and $\underline{\mathbb{P}}_{C}^{\text {reach }}$, respectively (Def. 2.2 ). In this section we use $\mathbb{K}=[0, \infty]$.

Definition 4.1 (NNRepSupM for pCFG). Let $\Gamma$ be a pCFG, $I$ be a pure invariant, and $C \subseteq I$ be a Borel set. An upper nonnegative repulsing supermartingale ( $U$ NNRepSupM) over $\Gamma$ for $C$ supported by $I$ is a function $\eta \in \mathcal{B}(I,[0, \infty])$ s.t.

i) $\eta(c) \geq 1$ for each $c \in C$, and $\quad$ ii) $\eta(c) \geq \overline{\mathbb{X}} \eta(c)$ for each $c \in I \backslash C$.

The function $\eta$ is a lower nonnegative repulsing supermartingale (L-NNRepSupM) if it satisfies the above conditions, but with $\overline{\mathbb{X}}$ replaced with $\underline{\mathbb{X}}$.

We shall prove soundness and completeness of NNRepSupM, based on the foundations in $\$ 2.3$. The following characterization is fundamental.

Proposition 4.2. In the setting of Def. 4.1 we define endofunctions $\overline{\Phi_{C}}$ and $\underline{\Phi_{C}}$ over $\mathcal{B}(I,[0, \infty])$ as follows:

$$
\overline{\Phi_{C}}(\eta)(x)=\left\{\begin{array}{ll}
1 & (x \in C) \\
(\overline{\mathbb{X}} \eta)(x) & (x \notin C),
\end{array} \quad \underline{\Phi_{C}}(\eta)(x)= \begin{cases}1 & (x \in C) \\
(\underline{\mathbb{X}} \eta)(x) & (x \notin C) .\end{cases}\right.
$$

Then the upper reachability probability $\overline{\mathbb{P}}_{C}^{\mathrm{reach}}: L \times \mathbb{R}^{V} \rightarrow[0, \infty] 5$ is the least fixed point (lfp) of $\overline{\Phi_{C}}$. Similarly, $\underline{P}_{C}^{\text {reach }}$ is the lfp of $\underline{\Phi_{C}}$.

Proof. (Sketch) We first need to show that $\overline{\mathbb{P}}_{C}^{\text {reach }}$ and $\underline{\mathbb{P}}_{C}^{\text {reach }}$ are Borel measurable. This is not very easy, as they are defined via supremum or infimum over uncountably many schedulers. We use the technique of $\varepsilon$-optimal scheduler known from control theory [5].

Checking that $\overline{\mathbb{P}}_{C}^{\text {reach }}$ and $\underline{\mathbb{P}}_{C}^{\text {reach }}$ are fixed points is not hard, though laborious. We use $\varepsilon$-optimal schedulers again for interchange between sup./inf. and integration.

Finally, the proofs for minimality differ for $\overline{\mathbb{P}}_{C}^{\text {reach }}$ and $\underline{\mathbb{P}}_{C}^{\text {reach }}$. For $\overline{\mathbb{P}}_{C}^{\text {reach }}$, we first observe that $\overline{\Phi_{C}}$ is $\omega$-continuous (immediate from Prop. 2.9). Therefore by the Kleene theorem, the lfp of $\overline{\Phi_{C}}$ is given by ${\overline{\Phi_{C}}}^{\omega}(\perp)$ (i.e. the chain in Thm. 2.4 stabilizes after $\omega$ steps). We can check the coincidence between $\bar{\Phi}_{C}{ }^{\omega}(\perp)$ and $\overline{\mathbb{P}}_{C}^{\text {reach }}$ by direct calculation.

For $\mathbb{P}_{C}^{\text {reach }}$, let $\eta$ be a fixed point of $\Phi_{C}$. Then for each $\varepsilon>0$, we can construct a scheduler $\sigma$ such that $\mathbb{P}_{C, \sigma}^{\text {reach }}(c) \sqsubseteq \eta(c) \overline{+} \varepsilon$ for each $c$ (at the $n$-th step, $\sigma$ chooses a $\varepsilon / 2^{n}$-optimal successor). Since $\underline{\mathbb{P}}_{C}^{\text {reach }}=\inf _{\sigma} \mathbb{P}_{C, \sigma}^{\text {reach }}$, this proves $\underline{\mathbb{P}}_{C}^{\text {reach }} \sqsubseteq \eta$.

It is easy to see that a U-NNRepSupM $\eta$ is nothing but a pre-fixed point of $\overline{\Phi_{C}}$ (i.e. $\overline{\Phi_{C}}(\eta) \sqsubseteq \eta$ ), and that an L-NNRepSupM $\eta$ is a pre-fixed point of $\Phi_{C}$. Therefore, soundness and completeness of NNRepSupM follow essentially from Cor. [2.5.

\footnotetext{
${ }^{4}$ We note that the theory of NNRepSupM can also be developed using Markov's lemma.

${ }^{5}$ Precisely it is the restriction of $\overline{\mathbb{P}}_{C}^{\text {reach }}$ to $I$; in what follows we do this identification for $\overline{\mathbb{P}}_{C}^{\text {reach }}$, $\underline{P}_{C}^{\text {reach }}, \overline{\mathbb{E}}_{C}^{\text {steps }}$, and $\underline{\mathbb{E}}_{C}^{\text {steps }}$.
} 
Corollary 4.3. 1. (Soundness) If $\eta$ is a U-NNRepSupM for $C$ supported by I, then for each $c \in I \backslash C$ we have $\overline{\mathbb{P}}_{C}^{\text {reach }}(c) \leq \eta(c)$.

Similarly, if $\eta$ is an L-NNRepSupM for C supported by $I$, then for each $c \in I \backslash C$ we have $\mathbb{P}_{C}^{\mathrm{reach}}(c) \leq \eta(c)$. This means, concretely, that for each $\varepsilon>0$ there is a scheduler $\sigma \in \operatorname{Sch}_{\Gamma}$ such that, for any $c \in I \backslash C$, we have $\mathbb{P}_{C, \sigma}^{\text {reach }}(c) \leq \eta(c)+\varepsilon$.

2. (Completeness) There exists a U-NNRepSupM $\eta$ that gives the optimal bound for $\overline{\mathbb{P}}_{C}^{\text {reach }}$. The same for L-NNRepSupM.

\section{Additive Ranking Supermartingales (ARnkSupM)}

We move on to the notion of additive ranking supermartingale (ARnkSupM) in Table 1 It is the best-known martingale-based notion for analysis of probabilistic programs and is used for overapproximating the expected reaching time. That its value is finite implies almost-sure reachability, too. We review its theory; the reason is to demonstrate that the same order-theoretic structure (see $\$ 2.3$ ) underlies ARnkSupM and NNRepSupM in the previous section. The completeness result ( (2) of Cor. 5.3) for pCFGs with real-valued variables seems new, too; See 88 for a detailed comparison to existing works. Proofs are done in a much similar manner to the ones in $\$$. In this section we use $\mathbb{K}=[0, \infty]$.

We note that completeness of U-ARnkSupM we state below is the one for strong almost-sure reachability [3]. U-ARnkSupM is incomplete for positive almost-sure reachability [15], that is, it cannot witness the condition $\forall \sigma . \mathbb{E}_{C, \sigma}^{\text {steps }}(c)<\infty$ in general.

Definition 5.1 (ARnkSupM for pCFG, [8] ). Let $\Gamma$ be a pCFG, $I \in \mathcal{B}\left(L \times \mathbb{R}^{V}\right)$ be a pure invariant, and $C \subseteq I$ be a Borel set. An upper additive ranking supermartingale ( $U$-ARnkSupM) over $\Gamma$ for $C$ supported by $I$ is a function $\eta \in \mathcal{B}(I,[0, \infty])$ that satisfies $\eta(c) \geq 1+\overline{\mathbb{X}} \eta(c)$ for each $c \in I \backslash C$.

The function $\eta$ is a lower additive ranking supermartingale (L-ARnkSupM) if it satisfies the above conditions, but with $\overline{\mathbb{X}}$ replaced with $\underline{\mathbb{X}}$.

Proposition 5.2. In the setting of Def. 5.1] we define endofunctions $\overline{\Psi_{C}}$ and $\underline{\Psi_{C}}$ over $\mathcal{B}(I,[0, \infty])$ as follows:

$$
\overline{\Psi_{C}}(\eta)(x)=\left\{\begin{array}{ll}
0 & (x \in C) \\
1+(\overline{\mathbb{X}} \eta)(x) & (x \notin C),
\end{array} \quad \underline{\Psi_{C}}(\eta)(x)= \begin{cases}0 & (x \in C) \\
1+(\underline{\mathbb{X}} \eta)(x) & (x \notin C) .\end{cases}\right.
$$

Then the upper expected reaching time $\overline{\mathbb{E}}_{C}^{\text {steps }}: L \times \mathbb{R}^{V} \rightarrow[0, \infty]$ is the lfp of $\overline{\Psi_{C}}$. Similarly, $\underline{\mathbb{E}}_{C}^{\text {steps }}$ is the lfp of $\underline{\Psi_{C}}$.

Corollary 5.3. 1. (Soundness, e.g. [2]) If $\eta$ is a U-ARnkSupM for C supported by I, then for each $c \in I \backslash C$ we have $\overline{\mathbb{E}}_{C}^{\text {steps }}(c) \leq \eta(c)$. In particular, for each $c \in I \backslash C$ that satisfies $\eta(c)<\infty$ we have $\mathbb{P}_{C}^{\text {reach }}(c)=1$.

Similarly, if $\eta$ is an L-ARnkSupM for $C$ supported by $I$, then for each $c \in I \backslash C$ we have $\underline{\mathbb{E}}_{C}^{\text {steps }}(c) \leq \eta(c)$. This means, concretely, that for each $\varepsilon>0$ there is a scheduler $\sigma \in \operatorname{Sch}_{\Gamma}$ such that, for any $c \in I \backslash C$, we have $\mathbb{E}_{C, \sigma}^{\text {steps }}(c) \leq \eta(c)+\varepsilon$. In particular, for each $c \in I \backslash C$ that satisfies $\eta(c)<\infty$ we have $\overline{\mathbb{P}}_{C}^{\text {reach }}(c)=1$.

2. (Completeness) There exists a U-ARnkSupM $\eta$ that gives the optimal bound for $\overline{\mathbb{E}}_{C}^{\text {steps }}$. The same holds for L-ARnkSupM. 


\section{$6 \gamma$-Scaled Submartingales $(\gamma$-SclSubM)}

Here we present the theory of $\gamma$-scaled submartingales $(\gamma$-SclSubM). It is for underapproximating reachability (Table 1). Compared to the well-known method of ARnkSupM, the greatest advantage is in quantitative reasoning: the value of a $\gamma-\mathrm{SclSubM}$ is guaranteed to be below the reachability probability (which can be less than 1), while ARnkSupM is useful only if almost reachability holds. In this section we use $\mathbb{K}=[0,1]$.

The notion of $\gamma-\mathrm{SclSubM}$ is first introduced in [33], as an instance of a categorical abstraction of ranking functions. The current paper's contribution lies in the following: i) the theoretical developments about $\gamma$-SclSubM in concrete (non-categorical) terms; ii) introduction of nondeterminism (the setting of [33] is purely probabilistic); and iii) template-based synthesis of $\gamma$-SclSubM.

Definition 6.1 ( $\gamma$-SclSubM for pCFG, [33]). Let $\gamma \in(0,1)$ be given. An upper $\gamma$-Scaled Submartingale $(U-\gamma-S c l S u b M)$ over $\Gamma$ for $C$ supported by $I$ is a function $\eta \in \mathcal{B}(I,[-\infty, 1])$ that satisfies $\eta(c) \leq \gamma \cdot \overline{\mathbb{X}} \eta(c)$ for each $I \backslash C$. A lower $\gamma-$ Scaled Submartingale $(L-\gamma-S c l S u b M)$ over $\Gamma$ for $C$ supported by $I$ is a function $\eta \in \mathcal{B}(I,[-\infty, 1])$ that satisfies $\eta(c) \leq \gamma \cdot \underline{\mathbb{X}} \eta(c)$ for each $I \backslash C$.

The derivation of $\gamma$-SclSubM, from a categorical account in [33], can be described in the following concrete terms. A $\gamma-\mathrm{SclSubM}$ is a post-fixed point of certain functions (namely $\gamma \cdot \overline{\Phi_{C}}$ and $\gamma \cdot \underline{\Phi_{C}}$ below). According to (gfp-KT) in Cor. $2.5, \gamma$-SclSubM underapproximates a greatest fixed point-but reachability is a least fixed point. The trick here is as follows: 1$)$ thanks to the scaling by $\gamma \in(0,1)$, the gfp and lfp of $\gamma \cdot \overline{\Phi_{C}}$ coincide; and 2) the lfp (hence the gfp) of $\gamma \cdot \overline{\Phi_{C}}$ is easily seen to be below the lfp of $\overline{\Phi_{C}}$, that is, the reachability probability that we are after. The overall argument signifies the role of the Knaster-Tarski theorem.

Proposition 6.2. Let $\overline{\Phi_{C}}$ and $\underline{\Phi_{C}}$ be as defined in Prop. 4.2 Define endofunctions $\gamma \cdot \overline{\Phi_{C}}$ and $\gamma \cdot \underline{\Phi_{C}}$ over $\mathcal{B}(I,[0,1])$ as follows: $\left(\gamma \cdot \overline{\Phi_{C}}\right)(\eta)(x)=\left\{\begin{array}{ll}1 & (x \in C) \\ \gamma \cdot(\overline{\mathbb{X}} \eta)(x) & (x \notin C),\end{array}\right.$ and $\left(\gamma \cdot \underline{\Phi_{C}}\right)(\eta)(x)=\left\{\begin{array}{ll}1 & (x \in C) \\ \gamma \cdot(\underline{\mathbb{X}} \eta)(x) & (x \notin C) .\end{array}\right.$ Then we have i) $\mu\left(\gamma \cdot \overline{\Phi_{C}}\right) \sqsubseteq \mu \overline{\Phi_{C}}$ and $\mu\left(\gamma \cdot \underline{\Phi_{C}}\right) \sqsubseteq \mu \underline{\Phi_{C}}$, and ii $) \nu\left(\gamma \cdot \overline{\Phi_{C}}\right)=\mu\left(\gamma \cdot \overline{\Phi_{C}}\right)$ and $\nu\left(\gamma \cdot \underline{\Phi_{C}}\right)=\mu\left(\gamma \cdot \underline{\Phi_{C}}\right)$.

Corollary 6.3 (soundness). If $\eta$ is a $U$ - $\gamma$-SclSubM for $C$ supported by $I$, then for each $c \in I \backslash C$ we have $\overline{\mathbb{P}}_{C}^{\text {reach }}(c) \geq \eta(c)$. This means, concretely, that for each $\varepsilon>0$ there is a scheduler $\sigma \in \mathrm{Sch}_{\Gamma}$ such that, for any $c \in I \backslash C$, we have $\mathbb{P}_{C, \sigma}^{\text {reach }}(c) \geq \eta(c)-\varepsilon$.

Similarly, if $\eta$ is an $L-\gamma$-SclSubM for $C$ supported by $I$, then for each $c \in I \backslash C$ we have $\underline{\mathbb{P}}_{C}^{\text {reach }}(c) \geq \eta(c)$.

Proof. Just notice that if $\eta$ is an upper- or lower- $\gamma-\operatorname{SclSubM}$, then so is $\max \{\mathbf{0}, \eta\}$. The rest is as described in the paragraph before Prop. 6.2

\section{Implementation and Experiments}

We implemented template-based automated synthesis algorithms for NNRepSupM (\$4) and $\gamma$-SclSubM ( 6 , and present some experimental results. We implemented the following programs: 
I. synthesis of a U-NNRepSupM for an APP based on a linear template.

II. synthesis of a U-NNRepSupM for a PPP based on a polynomial template.

III. synthesis of an L- $\gamma$-SclSubM for an APP based on a linear template.

Each algorithm first translates given an APP or a PPP to a pCFG $\Gamma$ and a terminal configuration $C$, and then solves an optimization problem of finding a U-NNRepSupM (L- $\gamma$-SclSubM) over $\Gamma$ for $C$ that gives a small (large) value as possible at the initial configuration. Reduction of optimization problems to LP or SDP ones are done in standard ways in the literature; we use Farkas' lemma (see e.g. [8|13]) for the case of APPs, and Schmüdgen's Positivstellensatz (see e.g. [119]) for PPPs.

We have augmented the syntax of APPs and PPPs ( $\$ 2.2)$ so that we can specify an invariant $I$ and a terminal configuration $C$. The program does not synthesize an invariant nor prove the correctness of the given invariant, and therefore the user has to provide a correct invariant by hand or by using some algorithm, e.g. [22].

All the programs are implemented in OCaml. We have used glpk (v4.63) [16] and SDPT3 [29] for the LP and SDP solvers respectively. For the implementation of Prog. II] we have also made use of a MATLAB toolbox SOSTOOLS (v3.03) [30].

We tested our implementations for several APPs and PPPs. We have used different benchmark sets for Prog. 【【 and Prog. III because what is overapproximated by Prog. $\llbracket \llbracket\left(\overline{\mathbb{P}}_{C}^{\text {reach }}\right)$ and what is underapproximated by Prog. $\amalg\left(\mathbb{P}_{C}^{\text {reach }}\right)$ are different. The benchmarks implement the following probabilistic processes that are used as benchmarks in the literature. More details and codes are given in $\mathrm{G}$

(a). (Adversarial random walk) A variation of a random walk, whose analysis is more challenging because of additional adversarial nondeterministic choices [12]. We have considered three variants: (a-1) 1D, (a-2) 2D and (a-3) a variant of 2D. (a-1) is a random walk over $\mathbb{R}$ modeling a discrete queuing system, and is parametrized by $p_{1}, p_{2} \in[0,1]$ that determines the distribution of the number of packets that arrive in each round. (a-2) and (a-3) are random walks over $\mathbb{R}^{2}$ parametrized by $M_{1}, M_{2} \in$ $\mathbb{R}$. They determine the distribution of movement distances in each round. We added a queue size limit for (a-1) and a time limit for (a-2) and (a-3). If the queue size exceeds 10 in (a-1) or 100 rounds were consumed in (a-2) or (a-3), the program stops, and it is not counted as termination.

(b). (Room temperature control) A model of an air conditioning system for adjacent two rooms [1]9]. It is parametrized by real numbers $c$ and $p$ : the former determines the power of the air conditioner, and the latter determines the size of perturbation. We have also added a time limit of 100 as in above.

We have coded (a) (b) as an APP. Experiments for Prog. Iand III were carried out on a MacBook Pro laptop with a Core i5 processor $(2.6 \mathrm{GHz}, 2$ cores) and $16 \mathrm{GiB}$ RAM. That for Prog. [I] was carried out on an Amazon EC2 c4.large instance (May 2018, 2 vCPUs and 3.75 GiB RAM) running Ubuntu 16.04.4 LTS (64 bit). The results are in Table. 2 23 For each program, the first column ("time (s)") shows the total execution time, and the second column ("bound") shows the calculated probability bound.

(Applicability of NNRepSupM) Table 2 shows the results for Prog. 【一, the goal of these experiments is to certify the applicability of NNRepSupM to programs with 


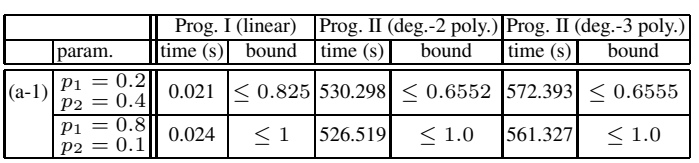

Table 2. Bounds by U-NNRepSupM

\begin{tabular}{|c|c|c|c|}
\hline & true reachability probability & U-NNRepSupM & 1 -RepSupM \\
\hline \hline (c-1 $)$ & $\frac{(0.4 / 0.6)^{5}-(0.4 / 0.6)^{10}}{1-(0.4 / 0.6)^{10}} \approx 0.116$ & 0.505 & $<1$ \\
\hline (c-2) & 0.5 & 0.5 & - \\
\hline (c-3) & $\int_{0}^{1}\left(\frac{0.25}{0.75}\right)^{\left[\log _{2}(1 / x) 7\right.} d x \approx 0.2$ & 0.5 & - \\
\hline (c-4) & $\left(\frac{0.25}{0.75}\right)^{1} \approx 0.333$ & - & $<1$ \\
\hline
\end{tabular}

Table 4. Probabilistic bounds given by U-NNRepSupM and $\varepsilon$-RepSupM

\begin{tabular}{|c|c|c|c|}
\hline & & Prog.LL & III) \\
\hline & Iparam. & time $(\mathrm{s}$ & \begin{tabular}{|l|} 
bound \\
\end{tabular} \\
\hline$(a-1)$ & $\begin{array}{l}p_{1}=0.2 \\
p_{2}=0.4\end{array}$ & 0.026 & $\geq 0$ \\
\hline & $\begin{array}{l}p_{1}=0.8 \\
p_{2}=0.1\end{array}$ & 0.022 & $\geq 0.751$ \\
\hline$(\mathrm{a}-2)$ & $\begin{array}{l}M_{1}=-1 \\
M_{2}=2\end{array}$ & 0.033 & $\geq 0$ \\
\hline & $\begin{array}{l}M_{1}=-2 \\
M_{2}=1\end{array}$ & 0.033 & $\geq 0.76$ \\
\hline$(\mathrm{a}-3)$ & $\begin{array}{l}M_{1}=-1 \\
M_{2}=2\end{array}$ & 0.028 & $\geq 0$ \\
\hline & $\begin{array}{l}M_{1}=-2 \\
M_{2}=1\end{array}$ & 0.040 & $\geq 0.80$ \\
\hline & $\begin{array}{l}c=0.1 \\
p=0.5\end{array}$ & 0.056 & $\geq 0$ \\
\hline (b) & $\begin{array}{l}c=0.1 \\
p=0.1\end{array}$ & 0.054 & $\geq 0.14$ \\
\hline
\end{tabular}

Table 3. Bounds by L- $\gamma-\mathrm{SclSubM}$ with $\gamma=0.999$

nondeterminism (a-1). We have tested them for (a-1) with two combinations of parameters. Prog. I- found a nontrivial bound for the reachability probability when $\left(p_{1}, p_{2}\right)=(0.2,0.4)$ while it failed to find such a bound when $\left(p_{1}, p_{2}\right)=(0.8,0.1)$. Intuitively, the random walk is more "unfavorable" in the former case in the sense that the opposite direction from a terminal configuration is chosen in higher probabilities. As expected, a polynomial NNRepSupM gives tighter bound than a linear one, but it took much longer. The bound was not improved by increasing the degree of the polynomial template.

(Applicability of $\gamma$-SclSubM) Table 3 shows the results for Prog. III, here we wish to certify applicability of our new method $\gamma$-SclSubM. For each variant of (a), we have tested Prog. III for two combinations of parameters. In each variant, Prog. III gives a nontrivial probability bound for one combination and a trivial bound for the other combination. In fact, all the cases where nontrivial bounds were "favorable" random walks where the direction to a terminal configuration tends to be chosen. In contrast, the cases where no nontrivial bound was found were "unfavorable" random walks. Note that this is the converse of the results for Prog. IIIII Prog. [III also succeeded in giving a nontrivial bound for (b) However, if we increase the parameter $c$ (i.e. if we strengthened the power of air conditioners), it failed to give a nontrivial bound.

(Comparison between NNRepSupM and $\varepsilon$-RepSupM) Both of NNRepSupM and $\varepsilon$ RepSupM (\$3) overapproximate $\overline{\mathbb{P}}_{C}^{\text {reach }}$. To compare them, we have also tested them for the following four simple pCFGs: (c-1) a bounded random walk over [0,10]; (c-2) a simple system with an infinite branching where $x$ is assigned a value taken from a geometric distribution; (c-3) a random walk over $[0,1]$ that exhibits geometric behaviors; and (c-4) an unbounded random walk. See $₫ \mathrm{H}$ for the concrete definitions of the pCFGs.

The results are shown in Table 4 The second column shows the true reachability probability obtained by hand calculation. The third and fourth columns show the probability bounds calculated by a linear NNRepSupM and a linear 1-RepSupM respectively.

For (c-1), both a linear NNRepSupM and a linear 1-RepSupM were found. However, while the NNRepSupM gave a non-trivial bound for the reachability probability, the probability bound calculated from the 1-RepSupM as in (1) was greater than 1 and 
hence trivial (cf. Thm. 3.2). Recall from Thm.3.212 that the 1-RepSupM can still refute almost-sure reachability. For (c-2) and (c-3), whose almost-sure reachability cannot be refuted by 1-RepSupMs, our algorithm found NNRepSupMs that give non-trivial probability bounds. In contrast, for (c-4), no NNRepSupM gave non-trivial bound while a 1-RepSupM that refutes almost-sure reachability was found.

\section{Related Work}

The notion of ranking supermartingale is first proposed by [8] aiming at extending applicability of quantitative invariants [24|23] to probabilistic programs with real-valued variables, but nondeterminism is not considered. Soundness of the method under demonic nondeterminism is studied in [2]11]15]; among them, lexicographic ranking supermartingales [2] can be seen as an extension of our U-ARnkSupM. Soundness under finite demonic/angelic nondeterminism is shown in [12].

Completeness of U-ARnkSupM for strong almost-sure termination [3] has previously been shown, but only in discrete settings [103]. The closest result to ours is [10], where they study pCFGs with demonic nondeterminism but restrict to integer-valued variables. Our proof that also works for real-valued variables utilizes $\varepsilon$-optimal schedulers from control theory [5].

Several under- and overapproximation methods for expected runtimes of probabilistic programs, which is defined inductively on its structure rather than on its semantics, is studied in [21]. Upper invariants of while-loops among them is a U-ARnkSupMlike notion in their setting. In [21] soundness and completeness of the upper invariant technique are derived from order-theoretic considerations. They handle probabilistic programs with demonic nondeterminism, but only discrete updates are allowed.

Probabilistic barrier certificates are studied in control theory [26]31] as a tool for overapproximating reachability. While it resembles our NNRepSupMs, their setting is purely probabilistic; we extend applicability of the technique to systems with nondeterminism.

$\varepsilon$-RepSupM [13] is also a technique for overapproximating reachability, which is studied for the purpose of synthesizing stochastic invariants. It is combined with ranking supermartingales to verify the persistence property of programs, too [9]13]. While there are certain similarities between $\varepsilon$-RepSupMs and NNRepSupMs, they are technically different because $\varepsilon$-RepSupMs exploit the $\kappa$-bounded differences condition, which is not assumed in our case. Their method is sound for refuting almost-sure reachability but does not provide nontrivial probability bound in general, and is not complete (see Fig. 3).

\section{Conclusions and Future Work}

We gave a comprehensive and comparative account of martingale-based techniques for approximating reachability probabilities. We demonstrated that several different approximation techniques-NNRepSupM, ARnkSupM, and $\gamma$-SclSubM- had a common structure of order-theoretic fixed points in their theory, while they originally arose from different communities. The key observation was that the reachability probability and the 
expected reaching time were the least fixed points of certain monotone endofunctions; soundness and completeness of the first two techniques are derived as its corollaries, and it is the basis for the proof of soundness of $\gamma$-SclSubM. We also implemented the techniques above and conducted experiments, of which results suggest the advantage of $\gamma$-SclSubM in quantitative reasoning, and the comparative advantage of NNRepSupM over $\varepsilon$-RepSupM in the quality of bounds.

In this paper we have focused on over- and underapproximating (i.e. refuting and verifying) reachability probabilities. For future work, we wish to study more complicated specifications such as recurrence $(\mathbf{G F} \varphi)$ and persistence $(\mathbf{F G} \varphi)$, too. Some martingale-based techniques have already been used (see e.g. [9]); we will investigate the use of lattice-theoretic progress measures, introduced in [18] as a generalization of progress measures for parity games [20], in the probabilistic settings.

Acknowledgment. We thank Shin-ya Katsumata, Takamasa Okudono and the anonymous referees for useful comments. The authors are supported by JST ERATO HASUO Metamathematics for Systems Design Project (No. JPMJER1603), the JSPS-INRIA Bilateral Joint Research Project "CRECOGI," and JSPS KAKENHI Grant Numbers $15 \mathrm{KT} 0012$ \& 15K11984. Natsuki Urabe is supported by JSPS KAKENHI Grant Number $16 \mathrm{~J} 08157$.

\section{References}

1. Abate, A., Katoen, J., Lygeros, J., Prandini, M.: Approximate model checking of stochastic hybrid systems. Eur. J. Control 16(6), 624-641 (2010)

2. Agrawal, S., Chatterjee, K., Novotný, P.: Lexicographic ranking supermartingales: an efficient approach to termination of probabilistic programs. PACMPL 2(POPL), 34:1-34:32 (2018)

3. Avanzini, M., Dal Lago, U., Yamada, A.: On probabilistic term rewriting. In: Gallagher, J.P., Sulzmann, M. (eds.) FLOPS 2018, Proceedings. Lecture Notes in Computer Science, vol. 10818, pp. 132-148. Springer (2018)

4. Baier, C., Katoen, J.P.: Principles of model checking. MIT Press (2008)

5. Bertsekas, D.P., Shreve, S.E.: Stochastic Optimal Control: The Discrete-Time Case. Athena Scientific (2007)

6. Bodík, R., Majumdar, R. (eds.): Proc. of POPL 2016. ACM (2016)

7. Boyd, S., Vandenberghe, L.: Convex Optimization. Cambridge University Press, New York, NY, USA (2004)

8. Chakarov, A., Sankaranarayanan, S.: Probabilistic program analysis with martingales. In: Sharygina, N., Veith, H. (eds.) Proc. of CAV 2013, Proceedings. LNCS, vol. 8044, pp. 511526. Springer (2013)

9. Chakarov, A., Voronin, Y., Sankaranarayanan, S.: Deductive proofs of almost sure persistence and recurrence properties. In: Chechik, M., Raskin, J. (eds.) Tools and Algorithms for the Construction and Analysis of Systems - 22nd International Conference, TACAS 2016, Proceedings. LNCS, vol. 9636, pp. 260-279. Springer (2016)

10. Chatterjee, K., Fu, H.: Termination of nondeterministic recursive probabilistic programs. CoRR abs/1701.02944 (2017), http://arxiv.org/abs/1701.02944

11. Chatterjee, K., Fu, H., Goharshady, A.K.: Termination analysis of probabilistic programs through Positivstellensatz's. In: Chaudhuri, S., Farzan, A. (eds.) Proc. of CAV 2016, Part I. LNCS, vol. 9779, pp. 3-22. Springer (2016) 
12. Chatterjee, K., Fu, H., Novotný, P., Hasheminezhad, R.: Algorithmic analysis of qualitative and quantitative termination problems for affine probabilistic programs. In: Bodík and Majumdar [6], pp. 327-342

13. Chatterjee, K., Novotný, P., Zikelic, D.: Stochastic invariants for probabilistic termination. In: Castagna, G., Gordon, A.D. (eds.) Proc. of POPL 2017. pp. 145-160. ACM (2017)

14. Cousot, R., Cousot, P.: Constructive versions of Tarski's fixed point theorems. Pacific Journal of Mathematics 82(1), 43-57 (1979)

15. Fioriti, L.M.F., Hermanns, H.: Probabilistic termination: Soundness, completeness, and compositionality. In: Rajamani, S.K., Walker, D. (eds.) Proceedings of the 42nd Annual ACM SIGPLAN-SIGACT Symposium on Principles of Programming Languages, POPL 2015, Mumbai, India, January 1517, 2015. pp. 489-501. ACM (2015). https://doi.org/10.1145/2676726.2677001. http://doi.acm.org/10.1145/2676726.2677001

16. The GNU linear programming kit, http://www.gnu.org/software/glpk

17. Gordon, A.D., Henzinger, T.A., Nori, A.V., Rajamani, S.K.: Probabilistic programming. In: Herbsleb, J.D., Dwyer, M.B. (eds.) Proceedings of the on Future of Software Engineering, FOSE 2014. pp. 167-181. ACM (2014)

18. Hasuo, I., Shimizu, S., Cîrstea, C.: Lattice-theoretic progress measures and coalgebraic model checking. In: Bodík and Majumdar [6], pp. 718-732

19. Horn, R.A., Johnson, C.R.: Matrix Analysis. Cambridge University Press, New York, NY, USA, 2nd edn. (2012)

20. Jurdzinski, M.: Small progress measures for solving parity games. In: Reichel, H., Tison, S. (eds.) STACS. LNCS, vol. 1770, pp. 290-301. Springer (2000)

21. Kaminski, B.L., Katoen, J., Matheja, C., Olmedo, F.: Weakest precondition reasoning for expected run-times of probabilistic programs. In: Thiemann, P. (ed.) Programming Languages and Systems - 25th European Symposium on Programming, ESOP 2016, Held as Part of the European Joint Conferences on Theory and Practice of Software, ETAPS 2016, Eindhoven, The Netherlands, April 2-8, 2016, Proceedings. Lecture Notes in Computer Science, vol. 9632, pp. 364-389. Springer (2016)

22. Katoen, J., McIver, A., Meinicke, L., Morgan, C.C.: Linear-invariant generation for probabilistic programs: - automated support for proof-based methods. In: Static Analysis - 17th International Symposium, SAS 2010. pp. 390-406 (2010)

23. McIver, A., Morgan, C.: Abstraction, Refinement And Proof For Probabilistic Systems (Monographs in Computer Science). SpringerVerlag (2004)

24. McIver, A., Morgan, C.: Developing and reasoning about probabilistic programs in $p G C L$. In: Cavalcanti, A., Sampaio, A., Woodcock, J. (eds.) Refinement Techniques in Software Engineering, First Pernambuco Summer School on Software Engineering, PSSE 2004, Revised Lectures. LNCS, vol. 3167, pp. 123-155. Springer (2004)

25. Nemirovski, A., Roos, C.: Motzkin transposition theorem (01 2008)

26. Prajna, S., Jadbabaie, A., Pappas, G.J.: Stochastic safety verification using barrier certificates. In: 2004 43rd IEEE Conference on Decision and Control. IEEE , Piscataway. pp. 929-934 (2004)

27. Scheiderer, C.: Positivity and Sums of Squares: A Guide to Recent Results, pp. 271-324. Springer New York, New York, NY (2009)

28. Schmüdgen, K.: Thek-moment problem for compact semi-algebraic sets. Mathematische Annalen 289(1), 203-206 (Mar 1991)

29. SDPT3, http://www.math.nus.edu.sg/ mattohkc/sdpt3.html

30. SOSTOOLS, http://sysos.eng.ox.ac.uk/sostools/

31. Steinhardt, J., Tedrake, R.: Finite-time regional verification of stochastic non-linear systems. I. J. Robotics Res. 31(7), 901-923 (2012) 
32. Tarski, A.: A Decision Method for Elementary Algebra and Geometry. Univ. of California Press, Berkeley (1951)

33. Urabe, N., Hara, M., Hasuo, I.: Categorical liveness checking by corecursive algebras. In: Proc. of LICS 2017. pp. 1-12. IEEE Computer Society (2017)

34. Vardi, M.Y.: An automata-theoretic approach to linear temporal logic. In: Moller, F., Birtwistle, G.M. (eds.) Banff Higher Order Workshop. Lecture Notes in Computer Science, vol. 1043, pp. 238-266. Springer (1995) 


\section{A Omitted Definitions in Section 2}

Definition A.1 (scheduler). A scheduler $\sigma$ of a pCFG is a pair $\left(\sigma_{t}, \sigma_{a}\right)$ of functions that satisfy the following.

- $\sigma_{t}$ maps each finite path $\pi$ that ends with a nondeterministic location $l$ (i.e. $\pi \in$ $\left.\left(L \times \mathbb{R}^{V}\right)^{*}\left(L_{N} \times \mathbb{R}^{V}\right)\right)$ to a probability distribution over $L$. We require that the support of $\sigma_{t}(\pi)$ is a subset of $\mapsto_{l}=\left\{l^{\prime} \mid l \mapsto l^{\prime}\right\}$. We further require that, for each $l^{\prime} \in L$, the function that maps a finite path $\pi$ to $\sigma_{t}(\pi)\left(\left\{l^{\prime}\right\}\right)$ is universally measurable, with respect to the canonical measurable structure on the set of finite paths that extends $\mathcal{B}(\mathbb{R})$.

- $\sigma_{a}$ maps each finite path $\pi$ that ends with an assignment location $l \in L_{A}$ for nondeterministic assignment (i.e. $\mathcal{U}(l) \in \mathcal{B}\left(\mathbb{R}^{n}\right)$ ) to a probability distribution over $\mathbb{R}$. We require that the support of $\sigma_{a}(\pi)$ is a subset of $\mathcal{U}(l)$. We further require that for each $A \in \mathcal{B}(\mathbb{R})$, the function that maps a finite path $\pi$ to $\sigma_{a}(\pi)(A)$ is universally measurable.

Definition A.2 (the map $\mu_{-}^{\sigma}$ ). For a given pCFG $\Gamma$, a scheduler $\sigma \in \operatorname{Sch}_{\Gamma}$ and a nonempty sequence $\pi \in\left(L \times \mathbb{R}^{V}\right)^{+}$of configurations, we define a probability measure $\mu_{\pi}^{\sigma} \in \mathcal{D}\left(L \times \mathbb{R}^{V}\right)$ as follows.

- For any $\pi \in\left(L \times \mathbb{R}^{V}\right)^{*}$ and $(l, \boldsymbol{x}) \in L \times \mathbb{R}^{V}$ such that $\pi(l, \boldsymbol{x})$ is a finite path,

- if $l \in L_{N}$ then $\mu_{\pi(l, \boldsymbol{x})}^{\sigma}=\Sigma_{l \mapsto l^{\prime}} \sigma_{t}(\pi(l, \boldsymbol{x}))\left(l^{\prime}\right) \cdot \delta_{\left(l^{\prime}, \boldsymbol{x}\right)}$,

- if $l \in L_{P}$ then $\mu_{\pi(l, \boldsymbol{x})}^{\sigma}=\Sigma_{l \mapsto l^{\prime}} \operatorname{Pr}_{l}\left(l^{\prime}\right) \cdot \delta_{\left(l^{\prime}, \boldsymbol{x}\right)}$,

- if $l \in L_{D}$ then $\mu_{\pi(l, \boldsymbol{x})}^{\sigma}=\delta_{\left(l^{\prime}, \boldsymbol{x}\right)}$, where $l^{\prime}$ is the unique location that satisfies $\boldsymbol{x} \models G\left(l, l^{\prime}\right)$,

- if $l \in L_{A}, \operatorname{succ}(l)=l^{\prime}$ and $\operatorname{Up}(l)=\left(x_{j}, u\right)$, then

$*$ if $u$ is a measurable function, then $\mu_{\pi(l, \boldsymbol{x})}^{\sigma}=\delta_{\left(l^{\prime}, \boldsymbol{x}\left(x_{j} \leftarrow u(\boldsymbol{x})\right)\right)}$,

$*$ if $u$ is a distribution, then $\mu_{\pi(l, x)}^{\sigma}$ is the unique measure that satisfies

$$
\forall A \in \mathcal{B}\left(L \times \mathbb{R}^{V}\right) \cdot\left(\mu_{\pi(l, \boldsymbol{x})}^{\sigma}\left(\left\{l^{\prime}\right\} \times A\right)=u(A)\right),
$$

* if $u$ is a measurable set, then $\mu_{\pi(l, \boldsymbol{x})}^{\sigma}$ is the unique measure that satisfies

$$
\forall A \in \mathcal{B}\left(L \times \mathbb{R}^{V}\right) .\left(\mu_{\pi(l, \boldsymbol{x})}^{\sigma}\left(\left\{l^{\prime}\right\} \times A\right)=\sigma_{a}(\pi(l, \boldsymbol{x}))(A)\right) .
$$

- For other cases, $\mu_{\pi(l, \boldsymbol{x})}^{\sigma}=\delta_{(l, \boldsymbol{x})}$.

\section{B Formal Definition for Syntax of Probabilistic Programs}

The following definitions are from [13]. We fix a countably infinite set $\mathcal{V}$ of variables. 
Definition B.1 (affine probabilistic program). An affine probabilistic program (APP) is a program defined by the following BNF notation:

$$
\begin{aligned}
& \langle s t m t\rangle::=\langle\text { assgn }\rangle \mid \text { skip } \mid\langle s t m t\rangle ;\langle\text { stmt }\rangle \\
& \mid \text { if }\langle n d b e x p r\rangle \text { then }\langle s t m t\rangle \text { else }\langle s t m t\rangle \text { fi } \\
& \mid \text { while }\langle\text { bexpr }\rangle \text { do }\langle s t m t\rangle \text { od } \\
& \langle\text { assgn }\rangle::=\langle\text { pvar }\rangle:=\langle\text { expr }\rangle \mid\langle\text { pvar }\rangle:=\operatorname{sample}(\langle\text { dist }\rangle) \\
& \mid\langle\text { pvar }\rangle:=\operatorname{ndet}(\langle\text { dom }\rangle) \\
& \langle\operatorname{expr}\rangle::=\langle\text { constant }\rangle \mid\langle\text { pvar }\rangle \mid\langle\text { constant }\rangle \cdot\langle\text { pvar }\rangle \mid\langle\exp r\rangle+\langle\text { expr }\rangle \\
& \mid\langle\operatorname{expr}\rangle-\langle\operatorname{expr}\rangle \\
& \langle\text { dom }\rangle::=\operatorname{Real} \mid \boldsymbol{R e a l}[\langle\text { const }\rangle,\langle\text { const }\rangle] \mid\langle\text { dom }\rangle \text { or }\langle\text { dom }\rangle \\
& \langle\text { bexpr }\rangle::=\langle\text { conjexpr }\rangle \mid\langle\text { conjexpr }\rangle \text { or }\langle\text { bexpr }\rangle \\
& \langle\text { conjexpr }\rangle::=\langle\text { literal }\rangle \mid\langle\text { literal }\rangle \text { and }\langle\text { conjexpr }\rangle \\
& \langle\text { literal }\rangle::=\langle\text { expr }\rangle \leq\langle\text { expr }\rangle \mid\langle\text { expr }\rangle \geq\langle\text { expr }\rangle \mid \neg\langle\text { literal }\rangle \\
& \langle\text { ndbexpr }\rangle::=\star|\operatorname{prob}(p)|\langle\text { bexpr }\rangle \quad \text { (where } p \in[0,1] \text { ) } \\
& \langle\text { pvar }\rangle::=v \in \mathcal{V} \quad\langle\text { dist }\rangle::=d \in \mathcal{D}(\mathbb{R}) \quad\langle\text { const }\rangle::=c \in \mathbb{R} .
\end{aligned}
$$

Definition B.2 (polynomial probabilistic program). A polynomial probabilistic program (PPP) is a program defined in almost the same manner as APP (Def.B.1) except that an expression $\langle\operatorname{expr}\rangle$ (2) in Def. B.1) is defined by the following BNF notation:

$\langle$ expr $\rangle::=\langle$ constant $\rangle \mid\langle$ pvar $\rangle \mid\langle$ expr $\rangle \cdot\langle\exp r\rangle|\langle\exp r\rangle+\langle\exp r\rangle|\langle\exp r\rangle-\langle\exp r\rangle$.

\section{Existence of an $\varepsilon$-optimal Scheduler}

In this section we show existence of $\varepsilon$-optimal schedulers for certain functions. For a given function $\varphi$ over pairs of schedulers and configurations, an $\varepsilon$-optimal scheduler $\sigma$ is the one for which the function $\varphi\left(\sigma,,_{-}\right)$is uniformly $\varepsilon$-close to $\sup _{\rho} \varphi\left(\rho,{ }_{-}\right)$. They resolve some measurability issues that arise in the proofs in $\$ 46$ We first fix some notations that simplify the description in later sections. In what follows, let $\Gamma$ be a pCFG, $I \in \mathcal{B}\left(L \times \mathbb{R}^{V}\right)$ be an invariant, $C \in \mathcal{B}(I)$, and $\bar{C}=I \backslash C$. Also for any $A \in \mathcal{B}\left(L \times \mathbb{R}^{V}\right)$, let $\chi_{A}$ be the characteristic function of $A$.

The reachability probability $\mathbb{P}_{C, \sigma}^{\mathrm{reach}=N}\left(c_{0}\right)$ in $N$ steps from a configuration $c_{0} \in I$ to a region $C \in \mathcal{B}(I)$ under a scheduler $\sigma \in \operatorname{Sch}_{\Gamma}$ is defined by $\mathbb{P}_{C, \sigma}^{\text {reach }=0}\left(c_{0}\right)=\chi_{C}\left(c_{0}\right)$, and for $N \geq 1$,

$\mathbb{P}_{C, \sigma}^{\mathrm{reach}=N}\left(c_{0}\right)=\chi_{\bar{C}}\left(c_{0}\right) \int_{I} \chi_{\bar{C}}\left(c_{1}\right) \mathrm{d} \mu_{c_{0}}^{\sigma} \ldots \int_{I} \chi_{\bar{C}}\left(c_{N-1}\right) \mathrm{d} \mu_{c_{0} \ldots c_{N-2}}^{\sigma} \int_{I} \chi_{C}\left(c_{N}\right) \mathrm{d} \mu_{c_{0} \ldots c_{N-1}}^{\sigma}$.

Also define the non-reaching probability $\mathbb{P}_{C, \sigma}^{\neg \text { reach } \leq N}\left(c_{0}\right)$ within $N$ steps from $c_{0}$ to $C$ under $\sigma$ by $\mathbb{P}_{C, \sigma}^{\neg \text { reach } \leq 0}\left(c_{0}\right)=\chi_{\bar{C}}\left(c_{0}\right)$, and for $N \geq 1$,

$\mathbb{P}_{C, \sigma}^{\neg \text { reach } \leq N}\left(c_{0}\right)=\chi_{\bar{C}}\left(c_{0}\right) \int_{I} \chi_{\bar{C}}\left(c_{1}\right) \mathrm{d} \mu_{c_{0}}^{\sigma} \ldots \int_{I} \chi_{\bar{C}}\left(c_{N-1}\right) \mathrm{d} \mu_{c_{0} \ldots c_{N-2}}^{\sigma} \int_{I} \chi_{\bar{C}}\left(c_{N}\right) \mathrm{d} \mu_{c_{0} \ldots c_{N-1}}^{\sigma}$. 
We omit the letter $I$ in descriptions of these functions (namely we do not write e.g. $\mathbb{P}_{I, C, \sigma}^{\text {reach }=N}$ ), as invariants just determine the domains of functions and do not affect their values at each configuration.

Definition C.1. For $\gamma \in(0,1]$, we define the following functions.

$$
\begin{aligned}
& \mathbb{P}_{C, \sigma}^{\text {reach } \leq N}=\sum_{i=0}^{N} \mathbb{P}_{C, \sigma}^{\text {reach }=i}, \quad \mathbb{E}_{C, \sigma}^{\text {steps } \leq N}=\left(\sum_{i=0}^{N} i \cdot \mathbb{P}_{C, \sigma}^{\text {reach }=i}\right)+N \cdot \mathbb{P}_{C, \sigma}^{\neg \text { reach } \leq N}, \\
& \mathbb{P}_{C, \gamma, \sigma}^{\text {reach } \leq N}=\sum_{i=0}^{N} \gamma^{i} \cdot \mathbb{P}_{C, \sigma}^{\text {reach }=i}, \quad \mathbb{P}_{C, \gamma, \sigma}^{\text {reach }}=\sum_{i \in \mathbb{N}} \gamma^{i} \cdot \mathbb{P}_{C, \sigma}^{\text {reach }=i} .
\end{aligned}
$$

For each of these functions, let an overline and an underline indicate the supremum and the infimum of the function over all possible configurations, respectively (e.g. $\overline{\mathbb{P}}_{C}^{\text {reach } \leq N}=\sup _{\sigma} \mathbb{P}_{C, \sigma}^{\text {reach } \leq N}$ and $\left.\underline{\mathbb{P}}_{C}^{\text {reach } \leq N}=\inf _{\sigma} \mathbb{P}_{C, \sigma}^{\text {reach } \leq N}\right)$.

By definition $\mathbb{P}_{C, 1, \sigma}^{\text {reach } \leq N}=\mathbb{P}_{C, \sigma}^{\text {reach } \leq N}, \overline{\mathbb{P}}_{C, 1}^{\text {reach } \leq N}=\overline{\mathbb{P}}_{C}^{\text {reach } \leq N}$ and $\underline{\mathbb{P}}_{C, 1}^{\text {reach } \leq N}=$ $\underline{\mathbb{P}}_{C}^{\text {reach } \leq N}$ hold, and similar coincidences also hold in the infinite horizon case. Now we are to introduce the definition of an $\varepsilon$-optimal scheduler and the lemma to be proved.

Definition C.2. Let $\varphi$ be a function from $I \times \operatorname{Sch}_{\Gamma}$ to $\mathbb{R} \cup\{ \pm \infty\}$, and $\varepsilon>0$. A scheduler $\sigma$ is $\varepsilon$-optimal for $\varphi$ if, for every $c \in I, \varphi(c, \sigma) \geq \sup _{\sigma^{\prime}} \varphi\left(c, \sigma^{\prime}\right)-\varepsilon$ holds when $\sup _{\sigma^{\prime}} \varphi\left(c, \sigma^{\prime}\right)<+\infty$, or $\varphi(c, \sigma) \geq \frac{1}{\varepsilon}$ holds otherwise.

Lemma C.3. Let a $p C F G \Gamma$, an invariant $I$, a set $C \in \mathcal{B}(I)$, a positive number $\varepsilon>0$ and a natural number $N$ be given. Let $\varphi: I \times \operatorname{Sch}_{\Gamma} \rightarrow \mathbb{R} \cup\{ \pm \infty\}$ be either of the following:

1. $\varphi(c, \sigma)= \pm \mathbb{P}_{C, \gamma, \sigma}^{\text {reach }}(c)(\gamma \in(0,1])$,

2. $\varphi(c, \sigma)= \pm \mathbb{P}_{C, \gamma, \sigma}^{\mathrm{reach} \leq N}(c)(\gamma \in(0,1])$,

3. $\varphi(c, \sigma)= \pm \mathbb{E}_{C, \sigma}^{\text {steps }}(c)$, or

4. $\varphi(c, \sigma)= \pm \mathbb{E}_{C, \sigma}^{\text {steps } \leq N}(c)$.

Then an $\varepsilon$-optimal scheduler $\sigma$ for $\varphi$ exists. Furthermore, they can be deterministic, i.e. $\sigma_{t}$ and $\sigma_{a}$ map any element in their domains to Dirac distributions.

To show this, we translate each pCFG to an infinite horizon stochastic optimal control model [5], for which existence of an $\varepsilon$-optimal scheduler (a.k.a. $\varepsilon$-optimal policy) is well known. The following is a slightly modified definition of the one in [5].

Definition C.4 ([5]). An (infinite horizon) stochastic optimal control model is a 7-tuple $\mathrm{SM}=\left(S_{\mathrm{SM}}, C_{\mathrm{SM}}, U_{\mathrm{SM}}, W_{\mathrm{SM}}, p, f, \alpha\right)$ which consists of the following.

- $S_{\mathrm{SM}}$ is a state space, a nonempty Borel space.

- $C_{\mathrm{SM}}$ is a control space, a nonempty Borel space. 
- $U_{\mathrm{SM}}$ is a control constraint, a function $U_{\mathrm{SM}}: S_{\mathrm{SM}} \rightarrow \mathcal{P}\left(C_{\mathrm{SM}}\right) \backslash\{\phi\}$ such that the set

$$
\operatorname{Graph}\left(U_{\mathrm{SM}}\right)=\left\{(x, u) \mid x \in S_{\mathrm{SM}}, u \in U_{\mathrm{SM}}(x)\right\}
$$

is analytic in $S_{\mathrm{SM}} \times C_{\mathrm{SM}}$.

- $W_{\mathrm{SM}}$ is a disturbance space, a nonempty Borel space.

- $p(d w \mid x, u)$ is a disturbance kernel, a Borel measurable stochastic kernel on $W_{\mathrm{SM}}$ given $S_{\mathrm{SM}} \times C_{\mathrm{SM}}$.

- $f$ is a system function, a Borel measurable function $f: S_{\mathrm{SM}} \times C_{\mathrm{SM}} \times W_{\mathrm{SM}} \rightarrow S_{\mathrm{SM}}$.

$-\alpha$ is a discount factor a positive real number.

A policy for SM is a sequence $\pi=\left(\mu_{i}\right)_{i \in \mathbb{N}}$, where $\mu_{i}\left(d u_{k} \mid x_{0}, u_{0}, \ldots, u_{i-1}, x_{i}\right)$ is a universally measurable stochastic kernel on $C_{\mathrm{SM}}$ given $\left(S_{\mathrm{SM}} \times C_{\mathrm{SM}}\right)^{i} \times S_{\mathrm{SM}}$ that satisfies

$$
\mu_{i}\left(U_{\mathrm{SM}}\left(x_{i}\right) \mid x_{0}, u_{0}, \ldots, u_{i-1}, x_{i}\right)=1
$$

for each $x_{0}, u_{0}, \ldots, u_{i-1}, x_{i}$. The set of all policies is denoted by $\Pi$.

For each natural number $N$, Borel distribution $p_{0}$ on $S_{\mathrm{SM}}$ and policy $\pi$, we have a unique probability measure $r_{N}\left(\pi, p_{0}\right)$ on $\left(S_{\mathrm{SM}} \times C_{\mathrm{SM}}\right)^{N}$ with the following condition; for each Borel set $B \subseteq\left(S_{\mathrm{SM}} \times C_{\mathrm{SM}}\right)^{N}$, the value of $r_{N}\left(\pi, p_{0}\right)(B)$ is the probability that the SM produces a run in $B$ under the initial distribution $p_{0}$ and the policy $\pi$. We can uniquely extend the collection $\left\{r_{N}\left(\pi, p_{0}\right)\right\}_{N \in \mathbb{N}}$ to the Borel measure $r\left(\pi, p_{0}\right)$ on $\left(S_{\mathrm{SM}} \times C_{\mathrm{SM}}\right)^{\omega}$ such that $r_{N}\left(\pi, p_{0}\right)(B)=r\left(\pi, p_{0}\right)([B])$ for each $B \in \mathcal{B}\left(\left(S_{\mathrm{SM}} \times\right.\right.$ $\left.\left.C_{\mathrm{SM}}\right)^{N}\right)$. For the concrete definition, see 9.1 in [5].

For a given $\mathrm{SM}$, we consider a one-stage cost function $g$, a lower-semianalytic function $g: \Gamma \rightarrow \mathbb{R} \cup\{ \pm \infty\}$ that is either nonnegative or nonpositive.

Definition C.5. Let $\pi$ be a policy for SM, and let a cost function $g$ and a natural number $N$ be given. The $N$-stage cost corresponding to $\pi \in \Pi$ at $x \in S_{\mathrm{SM}}$ is

$$
J_{N, \pi}(x)=\int\left[\sum_{k=0}^{N} \alpha^{k} g\left(x_{k}, u_{k}\right)\right] d r\left(\pi, \delta_{x}\right) .
$$

The infinite horizon cost corresponding to $\pi$ at $x$ is

$$
J_{\pi}(x)=\int\left[\sum_{k=0}^{\infty} \alpha^{k} g\left(x_{k}, u_{k}\right)\right] d r\left(\pi, \delta_{x}\right) .
$$

The $N$-stage optimal cost at $x$ is

$$
J_{N}^{*}(x)=\inf _{\pi \in \Pi} J_{\pi}(x) .
$$

The infinite horizon optimal cost at $x$ is

$$
J^{*}(x)=\inf _{\pi \in \Pi} J_{\pi}(x)
$$


We give a correspondence between pCFGs and stochastic control models as follows. Let $L_{A D}, L_{A P}$ and $L_{A N}$ be the set of all locations $l \in L_{A}$ such that $\mathcal{U}(l)$ is a Borel function, a Borel measure and a Borel set respectively. For a pCFG $\Gamma=\left(L, V, l_{\text {init }}, \boldsymbol{x}_{\text {init }}, \mapsto\right.$ , Up, $\operatorname{Pr}, G)$, let $\operatorname{SM}(\Gamma, \alpha)$ be the following stochastic control model:

- $S_{\mathrm{SM}}=L \times \mathbb{R}^{V}$

- $C_{\mathrm{SM}}=W_{\mathrm{SM}}=L \cup \mathbb{R}$

- $U_{\mathrm{SM}}=\left(\left(L_{D} \cup L_{P} \cup L_{A D} \cup L_{A P}\right) \times\{0\}\right) \cup\left(L_{D} \times L\right) \cup\left(L_{A N} \times \mathbb{R}\right)$

- $p$ is defined as follows:

- $p\left(\left\{l^{\prime}\right\} \mid(l, \boldsymbol{x}), \xi\right)=\operatorname{Pr}_{l}\left(\left\{l^{\prime}\right\}\right)$ and $p(\mathbb{R} \mid(l, \boldsymbol{x}), \xi)=0$ if $l \in L_{P}$ and $l^{\prime} \in L$

- $p(L \mid(l, \boldsymbol{x}), \xi)=0$ and $p(A \mid(l, \boldsymbol{x}), \xi)=\mathcal{U}(l)(A)$ if $l \in L_{A P}$ and $A \in \mathcal{B}(\mathbb{R})$

- $p(d w \mid(l, \boldsymbol{x}), \xi)=\delta_{0}$ otherwise

- $f$ is defined as follows:

- $f((l, \boldsymbol{x}), \xi, w)=\left(l^{\prime}, \boldsymbol{x}\right)$ if $l \in L_{D}$ and $l^{\prime}$ is the unique location such that $\boldsymbol{x} \models G\left(l, l^{\prime}\right)$

- $f((l, \boldsymbol{x}), \xi, w)=(w, \boldsymbol{x})$ if $l \in L_{P}$ and $w \in L$

- $f((l, \boldsymbol{x}), \xi, w)=(\xi, \boldsymbol{x})$ if $l \in L_{N}$ and $\xi \in L$

- $f((l, \boldsymbol{x}), \xi, w)=\left(l^{\prime}, \boldsymbol{x}\left(v_{j} \leftarrow \mathcal{U}(l)(\boldsymbol{x})\right)\right.$ if $l \in L_{A D}$ and $l \mapsto l^{\prime}$

- $f((l, \boldsymbol{x}), \xi, w)=\left(l^{\prime}, \boldsymbol{x}\left(v_{j} \leftarrow w\right)\right.$ if $l \in L_{A P}$ and $l \mapsto l^{\prime}$

- $f((l, \boldsymbol{x}), \xi, w)=\left(l^{\prime}, \boldsymbol{x}\left(v_{j} \leftarrow \xi\right)\right.$ if $l \in L_{A N}$ and $l \mapsto l^{\prime}$

- $f((l, \boldsymbol{x}), \xi, w)=(l, \boldsymbol{x})$ otherwise

$-\alpha \in(0,1]$.

For given $C \in \mathcal{B}\left(L \times \mathbb{R}^{V}\right)$, we consider the following cost functions:

$$
\begin{aligned}
& g_{1}((l, \boldsymbol{x}), \xi)=\left\{\begin{array}{ll}
1 & ((l, \boldsymbol{x}) \in C) \\
0 & ((l, \boldsymbol{x}) \notin C),
\end{array} \quad g_{2}((l, \boldsymbol{x}), \xi)= \begin{cases}-1 & ((l, \boldsymbol{x}) \in C) \\
0 & ((l, \boldsymbol{x}) \notin C),\end{cases} \right. \\
& g_{3}((l, \boldsymbol{x}), \xi)=\left\{\begin{array}{ll}
0 & ((l, \boldsymbol{x}) \in C) \\
1 & ((l, \boldsymbol{x}) \notin C),
\end{array} g_{4}((l, \boldsymbol{x}), \xi)= \begin{cases}0 & ((l, \boldsymbol{x}) \in C) \\
-1 & ((l, \boldsymbol{x}) \notin C) .\end{cases} \right.
\end{aligned}
$$

Without loss of generality we can assume that $\operatorname{SM}(\Gamma, \alpha)$ satisfies the following for any policy. When we use $g_{1}$ or $g_{2}$, any run of $\operatorname{SM}(\Gamma, \alpha)$ visits an element of $C$ at most once. When we use $g_{3}$ or $g_{4}$, any run of $\operatorname{SM}(\Gamma, \alpha)$ stays at the same state forever once it visits an element of $C$.

The Stochastic model $\operatorname{SM}(\Gamma, \alpha)$ is an interpretation of a $\mathrm{pCFG} \Gamma$ in the following sense. The proof is straightforward.

Proposition C.6. Let a $p C F G \Gamma$, an invariant $I \in \mathcal{B}\left(L \times \mathbb{R}^{V}\right)$ and a set $C \in I$ be given, and let $\operatorname{SM}(\Gamma, \alpha)$ be the stochastic control model defined as above. Then we have the following: if we take $g_{1}$ as the cost function, then we have the following.

1. For any scheduler $\sigma$ and a natural number $N$, there is a policy $\pi_{N}$ that satisfies $\mathbb{P}_{C, \alpha, \sigma}^{\mathrm{reach} \leq N}=J_{N, \pi_{N}}$, and vice versa. Thus in particular, we have $\mathbb{P}_{C, \alpha}^{\mathrm{reach} \leq N}=J_{N}^{*}$.

2. For any scheduler $\sigma$, there is a policy $\pi_{\infty}$ that satisfies $\mathbb{P}_{C, \alpha, \sigma}^{\mathrm{reach}}=J_{\pi_{\infty}}$, and vice versa. Thus in particular, we have $\underline{\mathbb{P}}_{C, \alpha}^{\text {reach }}=J^{*}$. 
In the same vein, we have:

$$
\begin{aligned}
& -\overline{\mathbb{P}}_{C, \alpha}^{\text {reach } \leq N}=J_{N}^{*} \text { and }-\overline{\mathbb{P}}_{C, \gamma}^{\text {reach }}=J^{*} \text { if the cost function is } g_{2} ; \\
& -\underline{\mathbb{E}}_{C}^{\text {steps } \leq N}=J_{N}^{*} \text { and } \underline{\mathbb{E}}_{C}^{\text {steps }}=J^{*} \text { if } \alpha=1 \text { and the cost function is } g_{3} ; \text { and } \\
& --\overline{\mathbb{E}}_{C}^{\text {steps } \leq N}=J_{N}^{*} \text { and }-\overline{\mathbb{E}}_{C}^{\text {steps }}=J^{*} \text { if } \alpha=1 \text { and the cost function is } g_{4} \text {. }
\end{aligned}
$$

Proof of Lem. C.3. For every case, take an $\varepsilon$-optimal policy $\pi$ for $\operatorname{SM}(\Gamma)$ that is given by [[5], Prop. 8.3 and Prop. 9.20], and then take the corresponding scheduler $\sigma$ that is given by Prop.C.6.

\section{Detail of Proofs in $84-6$}

In this section we provide detailed proofs of soundness and completeness of approximation methods in $\$ 46$ We first provide a set of lemmas that are used in the proofs. Through this section, fix a pCFG $\Gamma$, an invariant $I \in \mathcal{B}\left(L \times \mathbb{R}^{V}\right)$ and a set $C \in \mathcal{B}(I)$.

For given $\sigma \in \operatorname{Sch}_{\Gamma}$ and a finite path $\pi \in I^{+}$, let $\sigma_{\pi}=\left(\left(\sigma_{\pi}\right)_{t},\left(\sigma_{\pi}\right)_{a}\right)$ be any scheduler that satisfies $\left(\sigma_{\pi}\right)_{t}\left(\pi^{\prime}\right)=\sigma_{t}\left(\pi \pi^{\prime}\right)$ and $\left(\sigma_{\pi}\right)_{a}\left(\pi^{\prime}\right)=\sigma_{a}\left(\pi \pi^{\prime}\right)$ for each $\pi^{\prime} \in$ $I^{+}$such that $\pi \pi^{\prime}$ is a finite path. Notice that for each $\pi, \pi^{\prime} \in I^{+}$such that $\pi \pi^{\prime}$ is a finite path, $\left(\sigma_{\pi}\right)_{\pi^{\prime}}=\sigma_{\pi \pi^{\prime}}$ and $\mu_{\pi \pi^{\prime}}^{\sigma}=\mu_{\pi^{\prime}}^{\sigma_{\pi}}$ hold. For convenience, we assume $\sigma_{\pi}=\sigma$ if $\pi$ is an empty sequence.

Lemma D.1. Let $\mathbb{K}$ be a proper convex closed subset of $\mathbb{R} \cup\{ \pm \infty\}$ and $\eta \in \mathcal{B}(I, \mathbb{K})$. Then for any $c \in I$ we have $\overline{\mathbb{X}} \eta(c)=\sup _{\sigma} \int_{I} \eta \mathrm{d} \mu_{c}^{\sigma}$ and $\underline{\mathbb{X}} \eta(c)=\inf _{\sigma} \int_{I} \eta \mathrm{d} \mu_{c}^{\sigma}$.

Lemma D.2. For $c \in I$, we have $\mathbb{P}_{C, \sigma}^{\text {reach }=N+1}(c)=\chi_{\bar{C}} \int_{I} \mathbb{P}_{C, \sigma_{c}}^{\text {reach }}=N\left(c^{\prime}\right) \mu_{c}^{\sigma}\left(\mathrm{d} c^{\prime}\right)$.

Lemma D.3. Let $\mathbb{K}$ be a proper convex closed subset of $\mathbb{R} \cup\{ \pm \infty\}$ and suppose $\varphi: I \times \operatorname{Sch}_{\Gamma} \rightarrow \mathbb{K}$ satisfies $\varphi(\ldots, \sigma) \in \mathcal{B}(I, \mathbb{K})$ for each $\sigma \in \operatorname{Sch}_{\Gamma}$. Then we have $\sup _{\sigma} \int_{I} \varphi\left(c^{\prime}, \sigma_{c}\right) \mu_{c}^{\sigma}\left(\mathrm{d} c^{\prime}\right)=\sup _{\sigma} \sup _{\rho} \int_{I} \varphi\left(c^{\prime}, \rho\right) \mu_{c}^{\sigma}\left(\mathrm{d} c^{\prime}\right)$ and $\inf _{\sigma} \int_{I} \varphi\left(c^{\prime}, \sigma_{c}\right) \mu_{c}^{\sigma}\left(\mathrm{d} c^{\prime}\right)=$ $\inf _{\sigma} \inf _{\rho} \int_{I} \varphi\left(c^{\prime}, \rho\right) \mu_{c}^{\sigma}\left(\mathrm{d} c^{\prime}\right)$.

Lemma D.4. Let $\mathbb{K}$ be a proper convex closed subset of $\mathbb{R} \cup\{ \pm \infty\}$ and let $\varphi: I \times$ $\operatorname{Sch}_{\Gamma} \rightarrow \mathbb{K}$ be a function such that $\varphi(\ldots, \sigma) \in \mathcal{B}(I, \mathbb{K})$ for each $\sigma \in \operatorname{Sch}_{\Gamma}$. Further assume that for any $\varepsilon>0$ there is an $\epsilon$-optimal scheduler for $\varphi$. Then $\sup _{\sigma} \varphi(\ldots, \sigma) \in$ $\mathcal{B}(I, \mathbb{K})$ and for any $\mu \in \mathcal{D}(\mathbb{R})$ we have $\sup _{\sigma} \int_{I} \varphi(c, \sigma) \mathrm{d} \mu=\int_{I} \sup _{\sigma} \varphi(c, \sigma) \mathrm{d} \mu$.

Proof. We have $\sup _{\sigma} \varphi(c, \sigma)=\sup _{n} \varphi\left(c, \sigma^{(n)}\right)$, where $\sigma^{(n)}$ is an $2^{-n}$-optimal scheduler for $\varphi$ which can be chosen independently of $c$. This proves $\sup _{\sigma} \varphi(\ldots, \sigma) \in \mathcal{B}(I, \mathbb{K})$.

(LHS $\leq$ RHS) Take a sequence $\left\{\sigma^{(n)}\right\}_{n \in \mathbb{N}}$ such that $\int_{I} \varphi\left(c, \sigma^{(n)}\right) \mathrm{d} \mu \stackrel{n \rightarrow \infty}{\longrightarrow}$ LHS. Then we have LHS $=\sup _{n} \int_{I} \varphi\left(c, \sigma^{(n)}\right) \mathrm{d} \mu=\int_{I} \sup _{n} \varphi\left(c, \sigma^{(n)}\right) \mathrm{d} \mu \leq \mathrm{RHS}$.

(LHS $\geq$ RHS) For given $\varepsilon>0$, let $\sigma$ be an $\varepsilon$-optimal scheduler for $\varphi$. Then we have $\int_{I} \varphi(c, \sigma) \mathrm{d} \mu \geq \int_{I} \sup _{\sigma^{\prime}} \varphi\left(c, \sigma^{\prime}\right) \mathrm{d} \mu-\epsilon$. This proves LHS $\geq$ RHS.

Lemma D.4 also shows that if for any $\varepsilon>0$ there is an $\varepsilon$-optimal scheduler for $-\varphi$ instead, then we have $\inf _{\sigma} \int_{I} \varphi(c, \sigma) \mathrm{d} \mu=\int_{I} \inf _{\sigma} \varphi(c, \sigma) \mathrm{d} \mu$. 
Proposition D.5. Let $F: L \rightarrow L$ be a monotone endofunction on a lattice $L$. Then the following hold.

- Let $L$ be an $\omega$-cpo, i.e. any ascending countable chain in $L$ has the supremum. Let $F: L \rightarrow L$ be $\omega$-continuous, i.e. $\bigsqcup_{n<\omega} F\left(l_{n}\right)=F\left(\bigsqcup_{n<\omega} l_{n}\right)$ holds for any $\left\{l_{n}\right\}_{n \in \mathbb{N}}$. Then $\mu F$ exists, and $F l \leq l$ implies $\mu F \leq l$.

- Let $L$ be an $\omega^{\mathrm{op}}$-cpo, i.e. any descending countable chain in $L$ has the infimum. Let $F: L \rightarrow L$ be $\omega^{\mathrm{op}}$-continuous, i.e. $\prod_{n<\omega} F\left(l_{n}\right)=F\left(\prod_{n<\omega} l_{n}\right)$ holds for any $\left\{l_{n}\right\}_{n \in \mathbb{N}}$. Then $F l \leq l$ implies $\mu F \leq l$, assuming $\mu F$ exists.

Proof. For the $\omega$-continuous case, we have $\mu F=\bigsqcup_{n<\omega} F^{n}(\perp)$ from Kleene fixed point theorem. As we have $F^{n}(\perp) \leq l$ for any pre-fixed point $l$ of $F$ and $n \in \mathbb{N}$, we have $\mu F=\bigsqcup_{n<\omega} F^{n}(\perp) \leq l$.

For the $\omega^{\mathrm{oP}}$-continuous case, from the assumption we can derive $\prod_{n<\omega} F^{n} l \leq l$, and as $F$ is $\omega^{\text {op }}$-continuous, $\prod_{n<\omega} F^{n} l$ is a fixed point of $F$. Thus we have $\mu F \leq$ $\prod_{n<\omega} F^{n} l \leq l$.

Corollary D.6. Let $F: L \rightarrow L$ be a monotone endofunction on a lattice $L$. Then the following hold.

- If $L$ is an $\omega$-cpo and $F$ is $\omega$-continuous, then $F l \geq l$ implies $\nu F \geq l$, assuming $\nu F$ exists.

- If $L$ is an $\omega^{\mathrm{op}}$-cpo and $F$ is $\omega^{\mathrm{op}}$-continuous, then $\nu F$ exists, and $F l \geq l$ implies $\nu F \geq l$.

\section{D.1 Soundness/Completeness of UNNRepSupM}

Proof of Proposition 4.2 By Lemma C.3 we have $\overline{\mathbb{P}}_{C}^{\text {reach }}(c)=\sup _{n} \mathbb{P}_{\left(C, \sigma^{(n)}\right)}^{\text {reach }}(c)$, where $\sigma^{(n)}$ is an $2^{-n}$-optimal scheduler for $\varphi=\left((c, \sigma) \mapsto \mathbb{P}_{(C, \sigma)}^{\text {reach }}(c)\right)$ which can be chosen independently of $c$. This proves $\overline{\mathbb{P}}_{C}^{\text {reach }}$ is Borel measurable, and similarly we can show that $\underline{\mathbb{P}}_{C}^{\text {reach }}, \overline{\mathbb{P}}_{C}^{\text {reach } \leq N}$ and $\underline{\mathbb{P}}_{C}^{\text {reach } \leq N}$ are Borel measurable for each $N \in \mathbb{N}$.

$\left(\overline{\mathbb{P}}_{C}^{\text {reach }}=\mu \overline{\Phi_{C}}\right.$. $)$ We first show that $\overline{\mathbb{P}}_{C}^{\text {reach } \leq N+1}=\bar{\Phi}_{C} \overline{\mathbb{P}}_{C}^{\text {reach } \leq N}$ holds for each $N \in \mathbb{N}$. To show this, observe the following holds for any $c \in I$ :

$$
\begin{aligned}
& \mathbb{P}_{C, \sigma}^{\mathrm{reach} \leq N+1}(c)=\mathbb{P}_{C, \sigma}^{\mathrm{reach}=0}(c)+\sum_{i=1}^{N+1} \mathbb{P}_{C, \sigma}^{\mathrm{reach}=i}(c) \\
& =\chi_{C}(c)+\sum_{i=0}^{N} \chi_{\bar{C}}(c) \int_{I} \mathbb{P}_{C, \sigma_{c}}^{\mathrm{reach}=i}\left(c^{\prime}\right) \mu_{c}^{\sigma}\left(\mathrm{d} c^{\prime}\right) \\
& =\chi_{C}(c)+\chi_{\bar{C}}(c) \int_{I} \mathbb{P}_{C, \sigma_{c}}^{\mathrm{reach} \leq N}\left(c^{\prime}\right) \mu_{c}^{\sigma}\left(\mathrm{d} c^{\prime}\right) .
\end{aligned}
$$

From Lemma C.3, D.3 and D.4 we can derive

$$
\sup _{\sigma} \int_{I} \mathbb{P}_{C, \sigma_{c}}^{\mathrm{reach} \leq N}\left(c^{\prime}\right) \mu_{c}^{\sigma}\left(\mathrm{d} c^{\prime}\right)=\sup _{\sigma} \int_{I} \overline{\mathbb{P}}_{C}^{\mathrm{reach} \leq N}\left(c^{\prime}\right) \mu_{c}^{\sigma}\left(\mathrm{d} c^{\prime}\right) .
$$


Thus, taking supremum over $\operatorname{Sch}_{\Gamma}$ in (3), we have $\overline{\mathbb{P}}_{C}^{\text {reach } \leq N+1}=\bar{\Phi}_{C} \mathbb{P}_{C}^{\text {reach } \leq N}$.

Now observe $\overline{\Phi_{C}}(\perp)=\chi_{C}=\overline{\mathbb{P}}_{C}^{\text {reach } \leq 0}$ holds. Then we can inductively show that $\overline{\mathbb{P}}_{C}^{\text {reach } \leq N}=\bar{\Phi}_{C}^{N+1}(\perp)$ for each $N \in \mathbb{N}$, and hence $\overline{\mathbb{P}}_{C}^{\text {reach }}=\bar{\Phi}_{C}{ }^{\omega}(\perp)$ holds. As $\overline{\Phi_{C}}$ is $\omega$-continuous, we have $\overline{\mathbb{P}}_{C}^{\text {reach }}=\mu \overline{\Phi_{C}}$ via Kleene fixed point theorem.

$\left(\underline{\mathbb{P}}_{C}^{\text {reach }}=\mu \underline{\Phi_{C}}.\right)$ Taking limit of $N$ in $(3)$ we have

$$
\mathbb{P}_{C, \sigma}^{\text {reach }}(c)=\chi_{C}(c)+\chi_{\bar{C}}(c) \int_{I} \mathbb{P}_{C, \sigma_{c}}^{\text {reach }}\left(c^{\prime}\right) \mu_{c}^{\sigma}\left(\mathrm{d} c^{\prime}\right)
$$

and in a similar way to the upper case we obtain $\underline{\mathbb{P}}_{C}^{\text {reach }}=\underline{\Phi}_{C} \underline{\mathbb{P}}_{C}^{\text {reach }}$, i.e. $\underline{\mathbb{P}}_{C}^{\text {reach }}$ is a fixed point of $\underline{\Phi_{C}}$.

Now we show that for any fixed point $\eta$ of $\Phi_{C}$ and $\varepsilon>0$ there is a scheduler

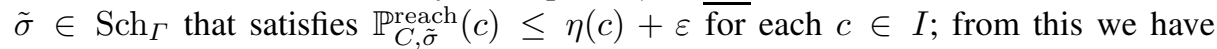
$\underline{\mathbb{P}}_{C}^{\text {reach }} \sqsubseteq \eta$ and hence $\underline{\mathbb{P}}_{C}^{\text {reach }}=\mu \underline{\Phi}_{C}$. For given a fixed point $\eta$ of $\underline{\Phi}_{C}$ and $\varepsilon>0$ define $\tilde{\sigma}$ as follows; for each finite path $\overline{\pi(l}, \boldsymbol{x}) \in I^{*}\left(I \cap L_{N} \times \mathbb{R}^{V}\right)$ we let $\tilde{\sigma}_{t}(\pi(l, \boldsymbol{x}))=\delta_{\tilde{l}}$, where $\tilde{l}$ satisfies $\eta(\tilde{l}, \boldsymbol{x})=\min _{l \mapsto l^{\prime}} \eta\left(l^{\prime}, \boldsymbol{x}\right)$; and for each finite path $\pi(l, \boldsymbol{x}) \in I^{*}(I \cap$ $\left.L_{A N} \times \mathbb{R}^{V}\right)$ such that $\operatorname{Up}(l)=(\operatorname{succ}(l), u)$ we let $\tilde{\sigma}_{a}(\pi(l, \boldsymbol{x}))=\delta_{\tilde{y}}$, where $\tilde{y}$ satisfies $\eta\left(\operatorname{succ}(l), \boldsymbol{x}\left(x_{j} \leftarrow \tilde{y}\right)\right) \leq \inf _{y \in u} \eta\left(\operatorname{succ}(l), \boldsymbol{x}\left(x_{j} \leftarrow y\right)\right)+2^{-(|\pi|+1)} \varepsilon$. Observe that $\tilde{\sigma}$ is universally (actually Borel) measurable.

We show the following by induction on $N \in \mathbb{N}$ : for each $N \in \mathbb{N}, \pi \in I^{*}$ and $c \in I$ such that $\pi c$ is a finite path, we have

$$
\mathbb{P}_{C, \tilde{\sigma}_{\pi}}^{\mathrm{reach} \leq N}(c) \leq \eta(c)+\sum_{k=|\pi|}^{|\pi|+N-1} 2^{-(k+1)} \varepsilon
$$

The case of $N=0$ is immediate to show, as $\mathbb{P}_{C, \sigma}^{\text {reach } \leq 0}=\chi_{C}$ holds for any scheduler $\sigma$. For the step case, observe that $\chi_{C}(c)+\chi_{\bar{C}}(c) \int_{I} \eta \mathrm{d} \mu_{c}^{\tilde{\sigma}_{\pi}} \leq \eta(c)+2^{-(|\pi|+1)} \varepsilon$ holds: indeed, if $c \in \bar{C} \cap\left(\left(L_{N} \cup L_{A N}\right) \times \mathbb{R}^{V}\right)$ then it is derived from the construction of $\tilde{\sigma}$; otherwise the value is independent of a scheduler and we have (LHS) $=\underline{\Phi_{C}} \eta(c)=$ $\eta(c)<(\mathrm{RHS})$. Thus we have

$$
\begin{aligned}
\mathbb{P}_{C, \tilde{\sigma}_{\pi}}^{\mathrm{reach} \leq N+1}(c) & =\chi_{C}(c)+\chi_{\bar{C}}(c) \int_{I} \mathbb{P}_{C, \tilde{\sigma}_{\pi c}}^{\mathrm{reach} \leq N} \mathrm{~d} \mu_{c}^{\tilde{\sigma}_{\pi}} \\
& \leq \chi_{C}(c)+\chi_{\bar{C}}(c) \int_{I} \eta \mathrm{d} \mu_{c}^{\tilde{\sigma}_{\pi}}+\sum_{k=|\pi|+1}^{|\pi|+N} 2^{-(k+1)} \varepsilon \\
& \leq \eta(c)+\sum_{k=|\pi|}^{|\pi|+N} 2^{-(k+1)} \varepsilon
\end{aligned}
$$

Now the claim is shown to be true, and in particular we have $\mathbb{P}_{C, \tilde{\sigma}}^{\text {reach } \leq N}(c) \leq \eta(c)+$ $\sum_{k=1}^{N} 2^{-k} \varepsilon$. Letting $N \rightarrow \infty$ we have $\mathbb{P}_{C, \tilde{\sigma}}^{\text {reach }}(c) \leq \eta(c)+\varepsilon$.

Proof of Corollary 4.3$] \in \mathcal{B}(I,[0, \infty])$ is a UNNRepSupM (LNNRepSupM) if and only if it is a pre-fixed point of $\overline{\Phi_{C}}\left(\underline{\Phi_{C}}\right)$. Soundness follows from Proposition D.5. 
and completeness is nothing but the fact that $\overline{\mathbb{P}}_{C}^{\text {reach }}\left(\underline{\mathbb{P}}_{C}^{\text {reach }}\right)$ itself is a UNNRepSupM (LNNRepSupM).

\section{D.2 Soundness/Completeness of ARnkSupM}

Proof of Proposition 5.2 The proof is similar to the one of Proposition 4.2 so some details are omitted in the proof below. First ofserve that, by LemmaC.3, we can show that $\overline{\mathbb{E}}_{C}^{\text {steps }}, \underline{\mathbb{E}}_{C}^{\text {steps }}, \overline{\mathbb{E}}_{C}^{\text {steps } \leq N}$ and $\underline{\mathbb{E}}_{C}^{\text {steps } \leq N}$ are Borel measurable for each $N \in \mathbb{N}$.

$\left(\overline{\mathbb{E}}_{C}^{\text {steps }}=\mu \overline{\Psi_{C}}\right.$.) We first show that $\overline{\mathbb{E}}_{C}^{\text {steps } \leq N+1}={\overline{\Psi_{C} \mathbb{E}_{C}^{\text {steps }} \leq N}}^{\text {holds for each }}$ $N \in \mathbb{N}$. To show that, observe that $\mathbb{P}_{C, \sigma}^{\text {reach } \leq N}+\mathbb{P}_{C, \sigma}^{\neg \text { reach } \leq N}=\mathbf{1}$ holds for each $N \in \mathbb{N}$ and $\sigma \in \operatorname{Sch}_{\Gamma}$ (intuitively, it asserts that from any configuration in $I$ the pCFG either visits the region $C$ within $N$ steps, or does not visit $C$ within $N$ steps, with probability 1). Thus for any $c \in I$ we have

$$
\begin{aligned}
\mathbb{E}_{C, \sigma}^{\text {steps } \leq N+1}(c)= & \left(\sum_{i=0}^{N+1} \mathbb{P}_{C, \sigma}^{\text {reach }=i}(c) \cdot i\right)+\mathbb{P}_{C, \sigma}^{\neg \text { reach } \leq N+1}(c) \cdot(N+1) \\
= & \left(\sum_{i=1}^{N+1} \mathbb{P}_{C, \sigma}^{\text {reach }=i}(c)\right)+\mathbb{P}_{C, \sigma}^{\neg \text { reach } \leq N+1}(c) \\
& +\left(\sum_{i=0}^{N} \mathbb{P}_{C, \sigma}^{\text {reach }=i+1}(c) \cdot i\right)+\mathbb{P}_{C, \sigma}^{\neg \text { reach } \leq N+1}(c) \cdot N \\
= & \chi_{\bar{C}}(c)\left(1+\int_{I} \mathbb{E}_{C, \sigma_{c}}^{\text {steps } \leq N}\left(c_{1}\right) \mu_{c}^{\sigma}\left(d c_{1}\right)\right) .
\end{aligned}
$$

Thus, thanks to Lemma C.3 D.3 and D.4 taking supremum over $\operatorname{Sch}_{\Gamma}$ in (5) we have $\overline{\mathbb{E}}_{C}^{\text {steps } \leq N+1}={\overline{\Psi_{C} \mathbb{E}_{C}}}_{C}^{\text {steps } \leq N}$.

Now because $\perp=\overline{\mathbb{E}}_{C}^{\text {steps } \leq 0}$, we can inductively show that $\overline{\mathbb{E}}_{C}^{\text {steps } \leq N}={\overline{\Psi_{C}}}^{N}(\perp)$ for each $N \in \mathbb{N}$, and hence $\overline{\mathbb{E}}_{C}^{\text {steps }}={\overline{\Psi_{C}}}^{\omega}(\perp)$ holds. As $\overline{\Psi_{C}}$ is $\omega$-continuous, we have $\overline{\mathbb{E}}_{C}^{\text {steps }}=\mu \overline{\Psi_{C}}$ via Kleene fixed point theorem.

$\left(\underline{\mathbb{E}}_{C}^{\text {steps }}=\mu \underline{\Psi_{C}}\right.$.) Taking limit of $N$ in 5 we have

$$
\mathbb{E}_{C, \sigma}^{\text {steps }}(c)=\chi_{\bar{C}}(c)\left(1+\int_{I} \mathbb{E}_{C, \sigma_{c}}^{\text {steps }}\left(c^{\prime}\right) \mu_{c}^{\sigma}\left(\mathrm{d} c^{\prime}\right)\right),
$$

and in a similar way to the upper case we obtain $\underline{\mathbb{E}}_{C}^{\text {steps }}=\underline{\Psi}_{C} \underline{\mathbb{E}}_{C}^{\text {steps }}$, i.e. $\underline{\mathbb{E}}_{C}^{\text {steps }}$ is a fixed point of $\Psi_{C}$.

It can be shown that for a given fixed point $\eta$ of $\underline{\Psi_{C}}$ and $\varepsilon>0$, the scheduler $\tilde{\sigma} \in \operatorname{Sch}_{\Gamma}$ in Proposition 4.2 satisfies $\mathbb{E}_{C, \tilde{\sigma}}^{\text {steps }}(c) \leq \eta(c) \overline{+\varepsilon}$ for any $c \in I$; the proof is almost identical to the one in Proposition 4.2. This proves $\underline{\mathbb{E}}_{C}^{\text {steps }}=\mu \underline{\Psi}_{C}$.

Proof of Corollary 5.3 $\eta \in \mathcal{B}(I,[0, \infty])$ is a UARnkSupM (LARnkSupM) if and only if it is a pre-fixed point of $\overline{\Psi_{C}}\left(\underline{\Psi_{C}}\right)$. Soundness follows from Proposition D.5, and completeness is nothing but the fact that $\overline{\mathbb{E}}_{C}^{\text {steps }}\left(\underline{\mathbb{E}}_{C}^{\text {steps }}\right)$ itself is a UARnkSupM (LARnkSupM). 


\section{D.3 Soundness of $\gamma$-SclSubM}

Proof of Proposition 6.2 Similar to (3), we have the following equation:

$$
\mathbb{P}_{C, \sigma, \gamma}^{\mathrm{reach} \leq N+1}(c)=\chi_{C}(c)+\chi_{\bar{C}}(c) \cdot \gamma \int_{I} \mathbb{P}_{C, \sigma_{c}, \gamma}^{\mathrm{reach} \leq N}\left(c^{\prime}\right) \mu_{c}^{\sigma}\left(\mathrm{d} c^{\prime}\right)
$$

Thus, thanks to Lemma C.3, D.3 and D.4, taking supremum and infimum over $\mathrm{Sch}_{\Gamma}$ in (6) we have $\overline{\mathbb{P}}_{C, \gamma}^{\text {reach } \leq N+1}=\left(\gamma \cdot \overline{\Phi_{C}}\right) \overline{\mathbb{P}}_{C, \gamma}^{\text {reach } \leq N}$ and $\underline{\mathbb{P}}_{C, \gamma}^{\text {reach } \leq N+1}=\left(\gamma \cdot \underline{\Phi_{C}}\right) \underline{P}_{C, \gamma}^{\text {reach } \leq N}$ respectively. Thus we can inductively show that $\overline{\mathbb{P}}_{C, \gamma}^{\text {reach } \leq N}=\left(\gamma \cdot \overline{\Phi_{C}}\right)^{N+1}(\perp)$ and $\underline{\mathbb{P}}_{C, \gamma}^{\text {reach } \leq N}=\left(\gamma \cdot \underline{\Phi}_{C}\right)^{N+1}(\perp)$ holds for each $N \in \mathbb{N}$.

$\left(\mu\left(\gamma \cdot \overline{\Phi_{C}}\right) \sqsubseteq \mu \overline{\Phi_{C}}\right.$. We have $\overline{\mathbb{P}}_{C, \gamma}^{\text {reach }}=\left(\gamma \cdot \overline{\Phi_{C}}\right)^{\omega}(\perp)$. As $\gamma \cdot \overline{\Phi_{C}}$ is $\omega$-continuous, we have $\mu\left(\gamma \cdot \overline{\Phi_{C}}\right)=\left(\gamma \cdot \overline{\Phi_{C}}\right)^{\omega}(\perp)$, and from the definition we have $\overline{\mathbb{P}}_{C, \gamma}^{\text {reach }} \sqsubseteq \overline{\mathbb{P}}_{C}^{\text {reach }}$. This proves $\mu\left(\gamma \cdot \overline{\Phi_{C}}\right) \sqsubseteq \mu \overline{\Phi_{C}}$.

$$
\begin{aligned}
&\left(\mu\left(\gamma \cdot \underline{\Phi_{C}}\right) \sqsubseteq \mu \underline{\Phi_{C}} .\right) \text { Taking limit of } N \text { in (6) we have } \\
& \mathbb{P}_{C, \sigma, \gamma}^{\text {reach }}(c)=\chi_{C}(c)+\chi_{\bar{C}}(c) \cdot \gamma \int_{I} \mathbb{P}_{C, \sigma_{c}, \gamma}^{\text {reach }}\left(c^{\prime}\right) \mu_{c}^{\sigma}\left(\mathrm{d} c^{\prime}\right),
\end{aligned}
$$

and in a similar way to the finite horizon case we have $\underline{\mathbb{P}}_{C, \gamma}^{\text {reach }}=\left(\gamma \cdot \underline{\Phi_{C}}\right) \underline{\mathbb{P}}_{C, \gamma}^{\text {reach }}$, i.e. $\underline{\mathbb{P}}_{C, \gamma}^{\text {reach }}$ is a fixed point of $\left(\gamma \cdot \underline{\Phi_{C}}\right)$. In particular we have $\sup _{N<\omega} \underline{\mathbb{P}}_{C, \gamma}^{\text {reach } \leq N}=(\gamma$. $\left.\underline{\Phi}_{C}\right)^{\omega}(\perp) \sqsubseteq \underline{\mathbb{P}}_{C, \gamma}^{\text {reach }}$. On the other hand, for any $c \in I$ we have

$$
\begin{aligned}
\underline{\mathbb{P}}_{C, \gamma}^{\mathrm{reach}}(c) & =\inf _{\sigma}\left(\sum_{i \in \mathbb{N}} \gamma^{i} \cdot \mathbb{P}_{C, \sigma}^{\mathrm{reach}=i}(c)\right) \\
& \leq \inf _{\sigma}\left(\sum_{i=0}^{N-1} \gamma^{i} \cdot \mathbb{P}_{C, \sigma}^{\mathrm{reach}=i}(c)\right)+\gamma^{N} \leq \sup _{M<\omega} \underline{P}_{C, \gamma}^{\mathrm{reach} \leq M}(c)+\gamma^{N},
\end{aligned}
$$

thus letting $N \rightarrow \infty$ we have $\underline{\mathbb{P}}_{C, \gamma}^{\text {reach }} \sqsubseteq\left(\gamma \cdot \underline{\Phi}_{C}\right)^{\omega}(\perp)$, and hence $\left(\gamma \cdot \underline{\Phi}_{C}\right)^{\omega}(\perp)=\underline{\mathbb{P}}_{C, \gamma}^{\text {reach }}$ is a fixed point of $\gamma \cdot \underline{\Phi}_{C}$. This proves $\underline{\mathbb{P}}_{C, \gamma}^{\text {reach }}=\mu\left(\gamma \cdot \underline{\Phi_{C}}\right)$, and by definition $\underline{\mathbb{P}}_{C, \gamma}^{\text {reach }} \sqsubseteq$ $\underline{\mathbb{P}}_{C}^{\text {reach }}=\mu \underline{\Phi_{C}}$.

$\left(\mu\left(\gamma \cdot \overline{\Phi_{C}}\right)=\nu\left(\gamma \cdot \overline{\Phi_{C}}\right)\right.$.) Let $\eta$ be any fixed point of $\gamma \cdot \overline{\Phi_{C}}$. In what follows we show the following by induction on $N \in \mathbb{N}$ : for any $c \in I$ and $N \in \mathbb{N}$ we have $\eta(c) \leq \overline{\mathbb{P}}_{C, \gamma}^{\mathrm{reach} \leq N}(c)+\gamma^{N} \cdot \chi_{\bar{C}}(c)$. 
For the case of $N=0$ the inequality is true (it just asserts $\eta \sqsubseteq \mathbf{1}$ ). For the step case, we have

$$
\begin{aligned}
\eta(c) & =\left(\gamma \cdot \overline{\Phi_{C}}\right) \eta(c) \\
& \leq\left(\gamma \cdot \overline{\Phi_{C}}\right)\left(\overline{\mathbb{P}}_{C, \gamma}^{\mathrm{reach} \leq N}+\gamma^{N} \cdot \chi_{\bar{C}}\right)(c) \\
& =\sup _{\sigma}\left(\chi_{C}(c)+\chi_{\bar{C}}(c) \cdot \gamma \int \overline{\mathbb{P}}_{C, \gamma}^{\mathrm{reach} \leq N}\left(c^{\prime}\right)+\gamma^{N} \cdot \chi_{\bar{C}}\left(c^{\prime}\right) \mu_{c}^{\sigma}\left(\mathrm{d} c^{\prime}\right)\right) \\
& \leq \sup _{\sigma}\left(\chi_{C}(c)+\chi_{\bar{C}}(c) \cdot \gamma \int \overline{\mathbb{P}}_{C, \gamma}^{\mathrm{reach} \leq N}\left(c^{\prime}\right) \mu_{c}^{\sigma}\left(\mathrm{d} c^{\prime}\right)\right)+\gamma^{N+1} \cdot \chi_{\bar{C}}(c) \\
& =\left(\gamma \cdot \overline{\Phi_{C}}\right) \overline{\mathbb{P}}_{C, \gamma}^{\mathrm{reach} \leq N}(c)+\gamma^{N+1} \cdot \chi_{\bar{C}}(c) \\
& =\overline{\mathbb{P}}_{C, \gamma}^{\mathrm{reach} \leq N+1}(c)+\gamma^{N+1} \cdot \chi_{\bar{C}}(c) .
\end{aligned}
$$

This proves the claim, and thus we have $\eta \sqsubseteq \mu\left(\gamma \cdot \overline{\Phi_{C}}\right)$. Hence $\mu\left(\gamma \cdot \overline{\Phi_{C}}\right)$ is also the greatest fixed point of $\gamma \cdot \overline{\Phi_{C}}$, which is our claim.

$$
\left(\mu\left(\gamma \cdot \underline{\Phi_{C}}\right)=\nu\left(\gamma \cdot \underline{\Phi_{C}}\right) .\right) \text { Similar to the upper case. }
$$

Proof of Corollary 6.3 We prove the upper case only, as the proof of the lower case is identical to it. Notice that if $\eta$ is a U- $\gamma-\operatorname{SclSubM}$, then so is $\eta^{\prime}=\max \{\boldsymbol{0}, \eta\}$, and $\eta^{\prime}$ is U- $\gamma$-SclSubM if and only if it is a post-fixed point of $\gamma \cdot \overline{\Phi_{C}}$. By Corollary D.6 this implies $\eta^{\prime} \sqsubseteq \nu\left(\gamma \cdot \overline{\Phi_{C}}\right)$, and from Proposition 6.2 and Proposition 4.2 we have $\eta \sqsubseteq \eta^{\prime} \sqsubseteq \nu\left(\gamma \cdot \overline{\Phi_{C}}\right)=\mu\left(\gamma \cdot \overline{\Phi_{C}}\right) \sqsubseteq \mu \overline{\Phi_{C}}=\overline{\mathbb{P}}_{C}^{\text {reach }}$.

\section{E Automated Synthesis of Martingale-Based Certificates}

In this section we discuss template-based automated synthesis algorithms for NNRepSupM (\$) and $\gamma$-SclSubM (\$6) for a pCFG.

We use two types of templates: a linear template and a polynomial template. Linear template-based synthesis has been proposed for $\varepsilon$-RepSupM [13], ARnkSupM [8], etc. Polynomial template-based synthesis is found in ARnkSupM [119]. Those algorithms can be easily adopted for synthesis of NNRepSupM and $\gamma$-SclSubM.

We first introduce necessary notions. Let $V=\left\{x_{1}, \ldots, x_{|V|}\right\}$ be a finite set of variables. Linear expressions and polynomial expressions over $V$ are defined in the usual manner; see Def. E.1. We follow [13] and call linear inequalities linear constraints. Their conjunctions are linear conjunctive predicates, and a disjunction of the latter is called a linear predicate. See Def.E.2. The polynomial variations are similarly defined.

Definition E.1 (expression). A linear expression over $V$ is a formula defined by the following BNF notation: $\mathfrak{a}::=r_{1}|x| r_{2} \mathfrak{a} \mid \mathfrak{a}+\mathfrak{a}$ where $x \in V$ and $r_{1}, r_{2} \in \mathbb{R}$. We inductively define $\llbracket \mathfrak{a} \rrbracket: \mathbb{R}^{V} \rightarrow \mathbb{R}$ by $\llbracket r_{1} \rrbracket(c):=r_{1}, \llbracket x \rrbracket(c):=c(x), \llbracket r_{2} \mathfrak{a}_{1} \rrbracket(c):=$ $r_{2} \cdot \llbracket \mathfrak{a}_{1} \rrbracket(c)$ and $\llbracket \mathfrak{a}_{1}+\mathfrak{b}_{2} \rrbracket(c):=\llbracket \mathfrak{a}_{1} \rrbracket(c)+\llbracket \mathfrak{a}_{2} \rrbracket(c)$. A polynomial expression over $V$ is defined by: $\mathfrak{b}::=r_{1}|x| \mathfrak{b} \cdot \mathfrak{b} \mid \mathfrak{b}+\mathfrak{b}$. We define $\llbracket \mathfrak{b} \rrbracket: \mathbb{R}^{V} \rightarrow \mathbb{R}$ similarly to $\llbracket \mathfrak{a} \rrbracket$. 
Definition E.2 (constraint/(conjunctive) predicate). A linear constraint over $V$ is a formula $\mathfrak{a} \triangleright 0$ where $\triangleright \in\{\geq,>\}$ and $\mathfrak{a}$ is a linear expression over $V$. A linear conjunctive predicate over $V$ is a formula $\mathfrak{r}_{1} \wedge \cdots \wedge \mathfrak{r}_{m}$ where each $\mathfrak{r}_{i}$ is a linear constraint. A linear predicate over $V$ is a formula $\mathfrak{q}_{1} \vee \cdots \vee \mathfrak{q}_{n}$ where each $\mathfrak{q}_{i}$ is a linear conjunctive predicate. We define $\llbracket \mathfrak{a} \triangleright 0 \rrbracket, \llbracket \mathfrak{r}_{1} \wedge \cdots \wedge \mathfrak{r}_{m} \rrbracket, \llbracket \mathfrak{q}_{1} \vee \cdots \vee \mathfrak{q}_{n} \rrbracket \subseteq \mathbb{R}^{V}$ by $\llbracket \mathfrak{a} \triangleright 0 \rrbracket:=\{\boldsymbol{x} \mid \llbracket \mathfrak{a} \rrbracket(\boldsymbol{x}) \triangleright 0\}, \llbracket \mathfrak{r}_{1} \wedge \cdots \wedge \mathfrak{r}_{m} \rrbracket:=\left\{\boldsymbol{x} \mid \boldsymbol{x} \in \llbracket \mathfrak{r}_{1} \rrbracket \cap \cdots \cap \llbracket \mathfrak{r}_{m} \rrbracket\right\}$ and $\llbracket \mathfrak{q}_{1} \vee \cdots \vee \mathfrak{q}_{m} \rrbracket:=\left\{v \mid v \in \llbracket \mathfrak{q}_{1} \rrbracket \cup \cdots \cup \llbracket \mathfrak{q}_{n} \rrbracket\right\}$. Notions of polynomial constraint and (conjunctive) predicate are similarly defined.

Definition E.3 (expression/predicate map). A linear expression map over a pCFG $\Gamma$ is a tuple $\{\mathfrak{f}(l)\}_{l \in L}$ of linear expressions over $V$. Its semantics $\llbracket \mathfrak{f} \rrbracket: L \times \mathbb{R}^{V} \rightarrow \mathbb{R}$ is defined by $\llbracket \mathfrak{f} \rrbracket(l, \boldsymbol{x}):=\llbracket \mathfrak{f}(l) \rrbracket(\boldsymbol{x})$. A linear predicate map over $\Gamma$ is a tuple $\mathfrak{P}=$ $\{\mathfrak{P}(l)\}_{l \in L}$ of linear predicates over $V$. We write $\llbracket \mathfrak{P} \rrbracket$ for $\{(l, \boldsymbol{x}) \mid \boldsymbol{x} \in \llbracket \mathfrak{P}(l) \rrbracket\} \subseteq L \times$ $\mathbb{R}^{V}$. If each $\mathfrak{P}(l)$ is a linear conjunctive predicate, then it is called a linear conjunctive predicate map. Notions of polynomial expression map and polynomial (conjunctive) predicate map are similarly defined.

\section{E.1 Linear Template-Based Synthesis of NNRepSupM}

Assume we are given a pCFG $\Gamma=\left(L, V, l_{\text {init }}, \boldsymbol{x}_{\text {init }}, \mapsto, \operatorname{Pr}, G\right)$, and linear predicate maps $\mathfrak{C}$ and $\mathfrak{I}$ over $\Gamma$. We further assume that $\llbracket \mathfrak{C} \rrbracket \subseteq L \times \mathbb{R}^{V}$ is an invariant 6 . We consider a linear template-based algorithm that synthesizes a linear expression map $\mathfrak{f}=\{\mathfrak{f}(l)\}_{l \in L}$ such that $\llbracket \mathfrak{f} \rrbracket: L \times \mathbb{R}^{V} \rightarrow \mathbb{R}$ is an U-NNRepSupM (Def.4.1) over $\Gamma$ for $\llbracket \mathfrak{C} \rrbracket$ supported by $\mathfrak{I}$. In fact, it can be easily adopted from the ones in [813]. We hereby briefly sketch its overview. See $\$$ F.1 for a more detailed description. The algorithm only works for "linear" pCFGs in the following sense.

Assumption E.4. 1. For each $l \in L_{A}$ such that $\operatorname{Up}(l)=(v, u)$,

(a) if $u \in \mathcal{B}\left(\mathbb{R}^{V}, \mathbb{R}\right)$, then a linear expression $\mathfrak{a}$ over $V$ s.t. $u=\llbracket \mathfrak{a} \rrbracket$ is given;

(b) if $u \in \mathcal{D}(\mathbb{R})$, then its expectation $\mathbb{E} u \in \mathbb{R}$ is known; and

(c) if $u \in \mathcal{B}(\mathbb{R})$, then a linear predicate $\mathfrak{P}$ over $\left\{x_{v}\right\}$ s.t. $u=\llbracket \mathfrak{P} \rrbracket$ is given.

2. For each $l \in L_{P}$, the set $\left\{l^{\prime} \in L \mid l \mapsto l^{\prime}\right\}$ of successor states is finite.

3. For each $l \in L_{D}$ and $l^{\prime} \in \mapsto_{l}$, a linear conjunctive predicate $\mathfrak{b}$ over $V$ s.t. $G\left(l, l^{\prime}\right)=$ $\llbracket \mathfrak{b} \rrbracket$ is given.

4. $\mathfrak{C}$ is a linear conjunctive predicate map.

The algorithm is almost the same as the ones in [8]13]: it first fixes a linear template, a linear expression map $\mathfrak{f}$ whose coefficients are unknown, for an U-NNRepSupM. We then reduce synthesis of an U-NNRepSupM to a feasibility problem with a conjunction of formulas of a form $\forall \boldsymbol{x} \in \mathbb{R}^{V^{\prime}} . \varphi \Rightarrow \psi$ is satisfied. Here: $V^{\prime}$ is a set of variables including $V ; \varphi$ is a linear conjunctive predicate without unknown coefficients; and $\psi$ is a linear expression with unknown coefficients. The key lemma is Farkas' lemma (see e.g. [7]): it reduces the problem to a feasibility problem with a conjunction of linear constraints, which is solvable in polynomial time as an linear programming (LP) problem using an LP solver.

Theorem E.5. If Asm. E.4 is satisfied, existence of a linear U-NNRepSupM for $\Gamma$ for $\mathfrak{C}$ at $M$ supported by $\mathfrak{I}$ is decidable in polynomial time.

\footnotetext{
${ }^{6}$ Such an invariant can be generated using an existing method, e.g. [22].
} 
Remark E.6. By Cor. 4.3, if $\mathfrak{f}$ is an U-NNRepSupM, then $\overline{\mathbb{P}}_{\llbracket \mathfrak{C} \rrbracket}^{\text {reach }}\left(l_{\text {init }}, \boldsymbol{x}_{\text {init }}\right) \leq$ $\llbracket \mathfrak{f} \rrbracket\left(l_{\text {init }}, \boldsymbol{x}_{\text {init }}\right)$. We naturally want a better bound, and to this end, we have to minimize $\llbracket \mathfrak{f} \rrbracket\left(l_{\text {init }}, \boldsymbol{x}_{\text {init }}\right)$. This task is easy in the algorithm above: by setting $\llbracket \mathfrak{f} \rrbracket\left(l_{\text {init }}, \boldsymbol{x}_{\text {init }}\right)$, which is a linear expression over the set $U$ of unknown coefficients, to the objective function of the induced LP problem and ask the LP solver to minimize it, we can minimize the probability bound. Similar arguments hold for polynomial U-NNRepSupM and linear lower $\gamma$-SclSubM discussed in later sections (although in the latter case, we maximize the probability bound).

Remark E.7. The algorithm described above does not work for lower NNRepSupMs because if we similarly reduce the axioms of L-NNRepSupM, then the resulting formula can contain both conjunction and disjunction, and therefore it cannot be solved using an LP solver. (They are still decidable because of the decidability of the first-order theory over reals (see e.g. [32]).) An analogous discussion for ARnkSupM is found in [12].

\section{E.2 Polynomial Template-Based Synthesis of NNRepSupM}

We next fix a polynomial template for an U-NNRepSupM. In this case, we can relax $\mathfrak{C}$ and $\mathfrak{I}$ in $\$$ E.1 to polynomial predicate maps. Asm. E.4 can be also relaxed.

Assumption E.8. - Similar conditions to Asm. E.41a, 1c, 2, 4 where "linear" is replaced by "polynomial" hold.

- For each $l \in L_{A}$ such that $\operatorname{Up}(l)=(v, u)$ and $u$ is a distribution on $\mathbb{R}$ and $n \in \mathbb{N}$, its $n$-th moment $\int_{x \in \mathbb{R}} x^{n} \mathrm{~d} u \in \mathbb{R}$ is known.

The algorithm is adopted from [119]. We hereby sketch it. See $₫$ F.2 for a more detailed description. It first fixes a polynomial template $\mathfrak{f}$, a polynomial expression map with unknown coefficients. Then in a similar manner to the linear case, the axioms of UNNRepSupM are reduced to a feasibility problem. The differences from the linear case are that we use a theorem called Positivstellensatz instead of Farkas' lemma, and the resulting feasibility problem is not an LP problem but an SDP problem. Several variants are known for Positivstellensatz. Among them, we use a variant called Schmüdgen's Positivstellensatz.

\section{E.3 Linear Template-Based Synthesis of $\gamma$-SclSubM}

The linear template-based algorithm for synthesizing a $\gamma$-SclSubM is very similar to the one for U-NNRepSupM in $\$$ E.1 However, in this case, by a similar reason to Rem.E.7, we have to focus on lower $\gamma$-SclSubM. We also note that we first have to fix $\gamma \in(0,1)$. The bigger $\gamma$ we fix, the better bound we can obtain.

Theorem E.9. If Asm. E.4 is satisfied, existence of a linear lower $\gamma$-SclSubM for $\Gamma$ for $\mathfrak{C}$ at $M$ supported by $\mathfrak{I}$ is decidable in polynomial time.

\section{F Algorithm for Finding Sub-/Supermartingales}

\section{F.1 Linear Template-based Algorithm for lower NNRepSupM and upper $\gamma-$ SclSubM}

We explain the algorithm that synthesize an upper L-NNRepSupM using a linear template. It is almost the same as the ones in [813]. The algorithm first fixes a linear 
template $\mathfrak{f}$ for an L-NNRepSupM as: $\mathfrak{f}(l)=a_{1}^{l} x_{1}+\cdots+a_{|V|}^{l} x_{|V|}+b^{l}$ where we let $V=\left\{x_{1}, \ldots, x_{|V|}\right\}$ and $a_{1}^{l}, \ldots, a_{|V|}^{l}, b^{l}$ are unknown coefficients for each $l \in L$. Let $U:=\left\{a_{1}^{l}, \ldots, a_{|V|}^{l}, b^{l} \mid l \in L\right\}$. We also fix a fresh variable $x_{|V|+1}$, and let $V^{\prime}:=V \cup\left\{x_{|V|+1}\right\}$ (this variable is used to deal with nondeterministic assignment). We then reduce the axioms of upper NNRepSupM to a predicate over variables in $V^{\prime}$ and unknown coefficients in $U$.

It results in a conjunction of constraints of a form $\forall \boldsymbol{x} \in \mathbb{R}^{V^{\prime}} . \varphi \Rightarrow \psi$, where

- $\varphi$ is a linear conjunctive predicate over $V^{\prime} 7$

- $\psi$ is a formula of a form $p_{1} x_{1}+\cdots+p_{\left|V^{\prime}\right|} x_{\left|V^{\prime}\right|}+q \geq 0$ where $p_{1}, \ldots, p_{\left|V^{\prime}\right|}, q$ are linear expressions over the set $U$ of unknown coefficients.

The concrete construction of those formulas is as follows:

Definition F.1 (cf. [12]13]). Let $\Gamma=\left(L, V, l_{\text {init }}, \boldsymbol{x}_{\text {init }}, \mapsto, \mathrm{Up}, \operatorname{Pr}, G\right)$ be a pCFG, I be a linear predicate map and $\mathfrak{C}$ be a linear conjunctive predicate map over $\Gamma$, and $M>0$. Assume $V=\left\{x_{1}, \ldots, x_{|V|}\right\}$. Let $x_{|V|+1}$ be a fresh variable such that $x_{|V|+1} \notin$ $V$, and let $V^{\prime}=V \cup\left\{x_{|V|+1}\right\}$. We define a set $U$ of unknown variables by $U:=$ $\bigcup_{l \in L}\left\{a_{1}^{l}, \ldots, a_{|V|}^{l}, b^{l}\right\}$. We write $\mathrm{Fml} \Rightarrow$ for the set of formulas of a form $\varphi \Rightarrow \psi$ where:

- $\varphi$ is a linear conjunctive predicate (Definition E.2) over $V^{\prime}$ all of whose inequalities are $\leq$; and

- $\psi$ is a formula of a form $p_{1} x_{1}+\cdots+p_{\left|V^{\prime}\right|} x_{\left|V^{\prime}\right|}+q \geq 0$ where $p_{1}, \ldots, p_{\left|V^{\prime}\right|}, q$ are linear expressions over $U$.

For such $\psi$, we define $\llbracket \psi \rrbracket \subseteq \mathbb{R}^{U} \times \mathbb{R}^{V^{\prime}}$ by $\llbracket \psi \rrbracket:=\left\{(u, v) \mid \llbracket p_{1} \rrbracket(u) v\left(x_{1}\right)+\cdots+\right.$ $\left.\llbracket p_{\left|V^{\prime}\right|} \rrbracket(u) v\left(x_{\left|V^{\prime}\right|}\right)+\llbracket q \rrbracket \geq 0\right\}$. For each $l \in L$, let $\Im(l, \boldsymbol{x})=\bigvee_{j=1}^{N_{l}^{\Im}} \bigwedge_{k=1}^{N_{l, j}^{\Im \prime}}\left(\alpha_{j, k}^{l} \triangleright 0\right)=$ $\bigvee_{j=1}^{N_{l}^{\mathcal{\jmath}}} \bigwedge_{k=1}^{N_{l, j}^{\Im \prime}}\left(c_{j, k, 1}^{l} x_{1}+\cdots+c_{j, k,|V|}^{l} x_{|V|}+d \triangleright 0\right)$ and $\mathfrak{C}(l, \boldsymbol{x})=\bigwedge_{i=1}^{N_{l}^{\mathfrak{c}}}\left(\beta_{i}^{l} \triangleright 0\right)$ where $\triangleright \in\{\geq,>\}$ and $\alpha_{j, k}^{l}$ and $\beta_{i}^{l}$ are linear expressions for each $l$. For each $l \in L$, we define $A_{1}^{l}, A_{2}^{l}, A_{3}^{l} \subseteq \mathrm{Fml}^{\Rightarrow}$ as follows:

- $A_{1}^{l}=\left\{\bigwedge_{k=1}^{N_{l, j}^{\Im \prime}}\left(\alpha_{j, k}^{l} \geq 0\right) \Rightarrow\left(a_{1}^{l} x_{1}+\cdots+a_{|V|}^{l} x_{|V|}+b^{l} \geq 0\right) \mid 1 \leq j \leq N_{l}^{\mathfrak{T}}\right\}$.

- $A_{2}^{l}=\left\{\bigwedge_{i=1}^{N_{l}^{\mathrm{e}}}\left(\beta_{i}^{l} \geq 0\right) \Rightarrow\left(a_{1}^{l} x_{1}+\cdots+a_{|V|}^{l} x_{|V|}+\left(b^{l}-M\right) \geq 0\right)\right\}$.

- $A_{3}^{l}$ is defined as follows.

- If $l \in L_{N}, A_{3}^{l}=\bigcup_{\left(l, l^{\prime}\right) \in \mapsto_{l}}\left\{\bigwedge_{k=1}^{N_{l, j}^{\gamma^{\prime}}(}\left(\alpha_{j, k}^{l} \geq 0\right) \wedge\left(-\beta_{i}^{l} \geq 0\right) \Rightarrow\left(\left(a_{1}^{l}-a_{1}^{l^{\prime}}\right) x_{1}+\right.\right.$ $\left.\left.\cdots+\left(a_{|V|}^{l}-a_{|V|}^{l^{\prime}}\right) x_{|V|}+\left(b^{l}-b^{l^{\prime}}\right) \geq 0\right) \mid 1 \leq i \leq N_{l}^{\mathfrak{c}}\right\}$.

- If $l \in L_{P}, A_{3}^{l}=\left\{\bigwedge_{k=1}^{N_{l, j}^{\Im \prime}}\left(\alpha_{j, k}^{l} \geq 0\right) \wedge\left(-\beta_{i}^{l} \geq 0\right) \Rightarrow\left(\left(a_{1}^{l}-\sum_{\left(l, l^{\prime}\right) \in \mapsto_{l}} \operatorname{Pr}_{l}\left(l, l^{\prime}\right)\right.\right.\right.$. $\left.a_{1}^{l^{\prime}}\right) x_{1}+\cdots+\left(a_{|V|}^{l}-\sum_{\left(l, l^{\prime}\right) \in \mapsto l} \operatorname{Pr}_{l}\left(l, l^{\prime}\right) \cdot a_{|V|}^{l^{\prime}}\right) x_{|V|}+\left(b^{l}-\sum_{\left(l, l^{\prime}\right) \in \mapsto l} \operatorname{Pr}_{l}\left(l, l^{\prime}\right)\right.$. $\left.\left.\left.b^{l^{\prime}}\right) \geq 0\right) \mid 1 \leq i \leq N_{l}^{\mathfrak{C}}\right\}$.

\footnotetext{
${ }^{7}$ Here we are relaxing a strict inequality $\mathfrak{a}>0$ to $\mathfrak{a} \geq 0$. This relaxation does not affect soundness of NNRepSupM, but it affects completeness. As was done for ARnkSupM in [8], if we use a generalized theorem called Motzkin's transposition theorem (see e.g. [25]) instead of Farkas' lemma, then such a relaxation is not necessary.
} 
- Let $l \in L_{D}$. For each $l^{\prime} \in \operatorname{succ}(l)$, assume $G\left(l, l^{\prime}\right)=\bigvee_{m=1}^{N_{l}^{G}} \bigwedge_{n=1}^{N_{l, m}^{G \prime}} G_{m, n}\left(l, l^{\prime}\right)$. We let: $A_{3}^{l}=\bigcup_{l^{\prime} \in \operatorname{succ}(l)}\left\{\bigwedge_{k=1}^{N_{l, j}^{3 \prime}}\left(\alpha_{j, k}^{l} \geq 0\right) \wedge\left(-\beta_{i}^{l} \geq 0\right) \wedge \bigwedge_{n=1}^{N_{l, m}^{G \prime}} G_{m, n}\left(l, l^{\prime}\right) \Rightarrow\right.$ $\left(\left(a_{1}^{l}-a_{1}^{l^{\prime}}\right) x_{1}+\cdots+\left(a_{|V|}^{l}-a_{|V|}^{l^{\prime}}\right) x_{|V|}+\left(b^{l}-b^{l^{\prime}}\right) \geq 0\right) \mid 1 \leq i \leq N_{l}^{\mathfrak{c}}, 1 \leq$ $\left.m \leq N_{l}^{G}\right\}$.

- Let $l \in L_{A}$ and $\operatorname{Up}(l)=(v, u) \in\{1, \ldots,|V|\} \times \mathcal{U}$. Note that there uniquely exists $l^{\prime} \in L$ such that $l \mapsto l^{\prime}$.

$*$ If $u$ is a measurable function $\mathbb{R}^{V} \rightarrow \mathbb{R}$ s.t. $u=\llbracket r_{1} x_{1}+\cdots+r_{|V|} x_{|V|}+r \rrbracket$ where $r_{1}, \ldots, r_{|V|}, r \in \mathbb{R}$, then $A_{3}^{l}=\left\{\bigwedge_{k=1}^{N_{l, j}^{\Im \prime}}\left(\alpha_{j, k}^{l} \geq 0\right) \wedge\left(-\beta_{i}^{l} \geq 0\right) \Rightarrow\right.$ $\left(\left(a_{1}^{l}-a_{1}^{l^{\prime}}-r_{1} a_{v}^{l^{\prime}}\right) x_{1}+\cdots+\left(a_{v-1}^{l}-a_{v-1}^{l^{\prime}}-r_{v-1} a_{v}^{l^{\prime}}\right) x_{v-1}+\left(a_{v}^{l}-r_{v} a_{v}^{l^{\prime}}\right) x_{v}+\right.$ $\left(a_{v+1}^{l}-a_{v+1}^{l^{\prime}}-r_{v+1} a_{v}^{l^{\prime}}\right) x_{v+1}+\cdots+\left(a_{|V|}^{l}-a_{|V|}^{l^{\prime}}-r_{|V|} a_{v}^{l^{\prime}}\right) x_{|V|}+\left(b^{l}-\right.$ $\left.\left.\left.b^{l^{\prime}}-r a_{v}^{l^{\prime}}\right) \geq 0\right) \mid 1 \leq i \leq N_{l}^{\mathfrak{C}}\right\}$.

* If $u$ is a distribution on $\mathbb{R}$ s.t. $\mathbb{E} u=r \in \mathbb{R}$, then $A_{3}^{l}=\left\{\bigwedge_{k=1}^{N_{l, j}^{3 \prime}}\left(\alpha_{j, k}^{l} \geq\right.\right.$ $0) \wedge\left(-\beta_{i}^{l} \geq 0\right) \Rightarrow\left(\left(a_{1}^{l}-a_{1}^{l^{\prime}}\right) x_{1}+\cdots+\left(a_{v-1}^{l}-a_{v-1}^{l^{\prime}}\right) x_{1}+a_{v}^{l} x_{1}+\left(a_{v+1}^{l}-\right.\right.$ $\left.\left.\left.a_{v+1}^{l^{\prime}}\right) x_{v+1}+\cdots+\left(a_{|V|}^{l}-a_{|V|}^{l^{\prime}}\right) x_{|V|}+\left(b^{l}-b^{l^{\prime}}-r a_{v}^{l}\right) \geq 0\right) \mid 1 \leq i \leq N_{l}^{\mathfrak{C}}\right\}$.

* Let $u$ be a set s.t. $u=\llbracket \mathfrak{P} \rrbracket$ for a linear predicate $\mathfrak{P}=\bigvee_{t=1}^{T} \Lambda_{t^{\prime}=1}^{T^{\prime}}\left(b_{t, t^{\prime}} x_{v}+\right.$ $b_{t, t^{\prime}}^{\prime} \geq 0$ ) over $\left\{x_{v}\right\}$ (see Asm. E.4). Then we let $A_{3}^{l}=\left\{\bigwedge_{k=1}^{N_{l, j}^{\Im, j}}\left(\alpha_{j, k}^{l} \geq\right.\right.$ $0) \wedge\left(-\beta_{i}^{l} \geq 0\right) \wedge \bigwedge_{t^{\prime}=1}^{T^{\prime}}\left(b_{t, t^{\prime}} x_{|V|+1}+b_{t, t^{\prime}}^{\prime} \geq 0\right) \Rightarrow\left(\left(a_{1}^{l}-a_{1}^{l^{\prime}}\right) x_{1}+\cdots+\right.$ $\left(a_{v-1}^{l}-a_{v-1}^{l^{\prime}}\right) x_{v-1}+a_{v}^{l} x_{v}+\left(a_{v+1}^{l}-a_{v+1}^{l^{\prime}}\right) x_{v+1}+\cdots+\left(a_{|V|}^{l}-a_{|V|}^{l^{\prime}}\right) x_{|V|}+$ $\left.\left.\left(-a_{v}^{l^{\prime}}\right) x_{|V|+1}+\left(b^{l}-b^{l^{\prime}}\right) \geq 0\right) \mid 1 \leq i \leq N_{l}^{\mathfrak{c}}, 1 \leq m \leq N_{l}^{u}, 1 \leq t \leq T\right\}$.

Proposition F.2. We assume the situation in Definition F.1 Then for $\boldsymbol{u} \in \mathbb{R}^{U}$, we have:

$$
\begin{aligned}
& \left(\forall \boldsymbol{x} \in \mathbb{R}^{V^{\prime}} . \forall l \in L . \forall(\varphi \Rightarrow \psi) \in \bigcup_{l \in L} A_{1}^{l} \cup A_{2}^{l} \cup A_{3}^{l} . \boldsymbol{x} \in \llbracket \varphi \rrbracket \Rightarrow(\boldsymbol{u}, \boldsymbol{x}) \in \llbracket \psi \rrbracket\right) \\
& \Rightarrow\left(\begin{array}{l}
\text { If we let } \mathfrak{f}=\left\{\boldsymbol{u}\left(a_{1}^{l}\right) \cdot x_{1}+\cdots+\boldsymbol{u}\left(a_{|V|}^{l}\right) \cdot x_{|V|}+b^{l}\right\}_{l \in L}, \\
\text { then } \llbracket \mathfrak{f} \rrbracket \text { is an upper NNRepSupM for } \llbracket \mathfrak{C} \rrbracket \text { at } M \text { supported by } \llbracket \Im \rrbracket
\end{array}\right) .
\end{aligned}
$$

Using matrices, we can rewrite formulas of a form $\forall \boldsymbol{x} \in \mathbb{R}^{V^{\prime}} . \varphi \Rightarrow \psi$ as follows:

$$
\forall \boldsymbol{x} \in \mathbb{R}^{V^{\prime}} . \boldsymbol{A} \boldsymbol{x} \leq \boldsymbol{b} \Rightarrow \boldsymbol{c}^{T} \boldsymbol{x} \leq d .
$$

Here $\boldsymbol{A} \in \mathbb{R}^{V^{\prime} \times m}$ is a matrix and $\boldsymbol{b} \in \mathbb{R}^{m}$ is a vector whose elements are real numbers, while $c$ is a vector and $d$ is a scalar whose elements are linear expressions over $U$. The goal is to find a valuation $U \rightarrow \mathbb{R}$ that makes (7) hold.

By Farkas' lemma (see e.g. [7]), if $\{\boldsymbol{x} \mid \boldsymbol{A x} \leq \boldsymbol{b}\}$ is not empty then the inequality (7) is satisfied if and only if there exists a column vector $\boldsymbol{y}=\left(y_{1}, \ldots, y_{m}\right)^{T} \geq 0$ over $\mathbb{R}$ such that $\boldsymbol{A}^{T} \boldsymbol{y}=\boldsymbol{c}$ and $\boldsymbol{b}^{T} \boldsymbol{y} \leq d$. Hence the problem is reduced to a satisfiability problem of linear inequalities over variables $\left\{y_{1}, \ldots, y_{m}\right\} \cup U$, which can be checked in polynomial time using a linear programming solver (LP solver). 


\section{F.2 Polynomial Template-based Algorithm for lower NNRepSupM and upper $\gamma$-SclSubM}

The algorithm almost the same as the ones [119]. The key is the use of a theorem called Positivstellensatz (see e.g. [27]). Several variants are known for Positivstellensatz, and three of them were used in [11]. Among them, we use a variant called Schmüdgen's Positivstellensatz.

We first fix a maximum degree $d \in \mathbb{N}$ of the template, and fix a polynomial template $\mathfrak{f}=\left\{\mathfrak{f}(l)=\sum_{h \in \mathcal{M}_{d}} a_{h}^{l} \cdot h\right\}_{l \in L}$ for an U-NNRepSupM. Here $\mathcal{M}_{d}$ denotes the set of monomials whose degrees are no greater than $d$, and each $a_{h}^{l}$ is an unknown coefficient. We let $U=\left\{a_{h}^{l} \mid l \in L, h \in \mathcal{M}_{d}\right\}$. In a similar manner to the linear case, we can reduce the axioms of U-NNRepSupM into a conjunction of predicates of a form $\forall \boldsymbol{x} \in \mathbb{R}^{V} . \varphi \Rightarrow \psi$, where $\varphi$ is a polynomial conjunctive predicate $\bigwedge_{i=1}^{m} \mathfrak{g}_{i} \geq 0$ over $V^{\prime}:=V+\left\{x_{|V|+1}\right\}$ and $\psi$ is a formula of a form $\mathfrak{g}=p_{1} h_{1}+\cdots+p_{t} h_{t} \geq 0$ with $p_{1}, \ldots, p_{t}$ being polynomial expressions over $U$, and $h_{1}, \ldots, h_{t}$ are monomials over $V^{\prime}$.

To find a valuation $U \rightarrow \mathbb{R}$ satisfying $\forall \boldsymbol{x} \in \mathbb{R}^{V^{\prime}} . \psi \Rightarrow \varphi$, we make use of a notion of sum-of-square polynomial expression. It is a polynomial expression of a form $\mathfrak{h}=\sum_{i=1}^{s} \mathfrak{i}_{i}^{2}$ for some polynomial expressions $\mathfrak{i}_{1}, \ldots, \mathfrak{i}_{s}$. It is easy to see that $\forall \boldsymbol{x} \in$ $\mathbb{R}^{V} . \llbracket \mathfrak{h} \rrbracket(\boldsymbol{x}) \geq 0$. Therefore if there exists a family $\left\{\mathfrak{h}_{w_{1} \ldots w_{m}}\right\}_{w_{1}, \ldots, w_{m} \in\{0,1\}}$ of sumof-square polynomial expressions s.t. $\mathfrak{g}=\sum_{w_{1}, \ldots, w_{m} \in\{0,1\}} \mathfrak{h}_{w_{1} \ldots w_{m}} \cdot \prod_{j=1}^{m} \mathfrak{g}_{i}^{w_{i}}$, then we have $\forall \boldsymbol{x} \in \mathbb{R}^{V} . \varphi \Rightarrow \psi$ (i.e. $\forall \boldsymbol{x} \in \mathbb{R}^{V} .\left(\bigwedge_{i=1}^{m} \mathfrak{g}_{i} \geq 0\right) \Rightarrow(\mathfrak{g} \geq 0)$ ).

Schmüdgen's Positivstellensatz tells us that under certain conditions, its converse holds.

Theorem F.3 ([28]). Let $\mathfrak{g}, \mathfrak{g}_{1}, \ldots, \mathfrak{g}_{m}$ be polynomial expressions over $V$. If $\llbracket \wedge_{i=1}^{m} \mathfrak{g}_{i} \geq$ $0 \rrbracket \subseteq \mathbb{R}^{V}$ is compact, then $\forall \boldsymbol{x} \in \llbracket \bigwedge_{i=1}^{m} \mathfrak{g}_{i} \geq 0 \rrbracket$. $\llbracket g \rrbracket(\boldsymbol{x})>0$ iff there exists a family $\left\{\mathfrak{h}_{w}\right\}_{w \in\{0,1\}^{m}}$ of sum-of-square polynomial expressions such that $\mathfrak{g}=\sum_{w \in\{0,1\}^{m}} \mathfrak{h}_{w}$. $\prod_{j=1}^{m} \mathfrak{g}_{i}^{w_{i}}$.

To ensure that a polynomial expression is sum-of-square, we can use its well-known characterization (see e.g. [19]): a polynomial expression $\mathfrak{h}$ whose degree is no greater than $2 k$ is sum-of-square iff there exists a positive semidefinite matrix $A \in \mathbb{R}^{\left|\mathcal{M}_{k}\right| \times\left|\mathcal{M}_{k}\right|}$ such that $\mathfrak{h}=\boldsymbol{y}_{k}^{T} A \boldsymbol{y}_{k}$, where $\boldsymbol{y}_{k}$ is the column vector whose components consist of the monomials in $\mathcal{M}_{k}$.

To summarize, to check existence of a valuation $U \rightarrow \mathbb{R}$ satisfying $\forall \boldsymbol{x} \in \mathbb{R}^{V} . \varphi \Rightarrow$ $\psi$, it suffices to fix a maximum degree $k \in \mathbb{N}$ for sum-of-square polynomial expressions, fix additional unknown parameters $U^{\prime}:=\left\{a_{w, i, j}^{l} \mid l \in L, w \in\{0,1\}^{m}, 1 \leq i, j \leq\right.$ $\left.\left|\mathcal{M}_{k}\right|\right\}$ and find a valuation $U \cup U^{\prime} \rightarrow \mathbb{R}$ that satisfies the following conditions:

- for each $w \in\{0,1\}^{m}$, the following matrix is positive semidefinite:

$$
A_{w}^{l}:=\left(\begin{array}{ccc}
a_{w, 1,1}^{l} & \ldots & a_{w, 1,\left|\mathcal{M}_{k}\right|+1}^{l} \\
\vdots & \ddots & \vdots \\
a_{w,\left|\mathcal{M}_{k}\right|+1,1}^{l} & \ldots & a_{w,\left|\mathcal{M}_{k}\right|+1,\left|\mathcal{M}_{k}\right|+1}^{l}
\end{array}\right) .
$$

- $\mathfrak{g}=\sum_{w \in\{0,1\}^{m}} \boldsymbol{y}_{k}^{T} A_{w} \boldsymbol{y}_{k} \cdot \prod_{j=1}^{m} \mathfrak{g}_{i}^{w_{i}}$ where $\boldsymbol{y}_{k}$ is defined as above. 
By comparing the coefficients appearing in the equality in the latter condition, we can obtain a family of linear equalities over $U \cup U^{\prime}$. Therefore polynomial template-based synthesis of an U-NNRepSupM is reduced to a semidefinite programming (SDP) problem, which is solvable using an SDP solver.

Conditioned polynomial constraints for U-NNRepSupM are analogous to Def. F.1. Conditioned linear/polynomial constraints for lower $\gamma$-SclSubM are similar.

\section{G Probabilistic Programs Used in Experiments}

We present codes of probabilistic programs $1 \quad\{0 \leq x\}$ while true do used in experiments in $\$ 7$ We have augmented $2 \quad\{0 \leq x\} \quad x:=x-1$ the syntax of APP and PPP (\$2.2) with two $3 \quad\{0 \leq x\}$ refute $(x \leq 0)$ components " $\{\ldots\}$ " and "refute" for specify- $4 \quad$ od ing an invariant $I$ and a terminal configuration $C$ respectively. An example of a program code is shown on the right. If we feed this code to Prog. Ifor example, then it underapproximates $\overline{\mathbb{P}}_{C}^{\text {reach }}(c)$ where the line 3 is reached when $x \leq 0$, under an assumption that $x \geq 0$ holds in each program location.

(a-1) (1D adversarial random walk [12]) This models a discrete queuing system that starts with 2 packets in the queue, and in each round 0,1 or 2 packets are queued in probability $p_{1}, p_{2}$ and $1-p_{1}-p_{2}$ respectively. Each packet is processed depending on its type that is nondeterministically chosen: if it is "urgent," then the packet is immediately processed in one round in probability $1 / 8$, but in probability $7 / 8$ it fails to process the packet and produces another packet. If the type is "standard," then it is processed using two rounds in probability 1 . The process terminates if queue gets empty, and aborts (it is not counted as termination) if the queue is full.

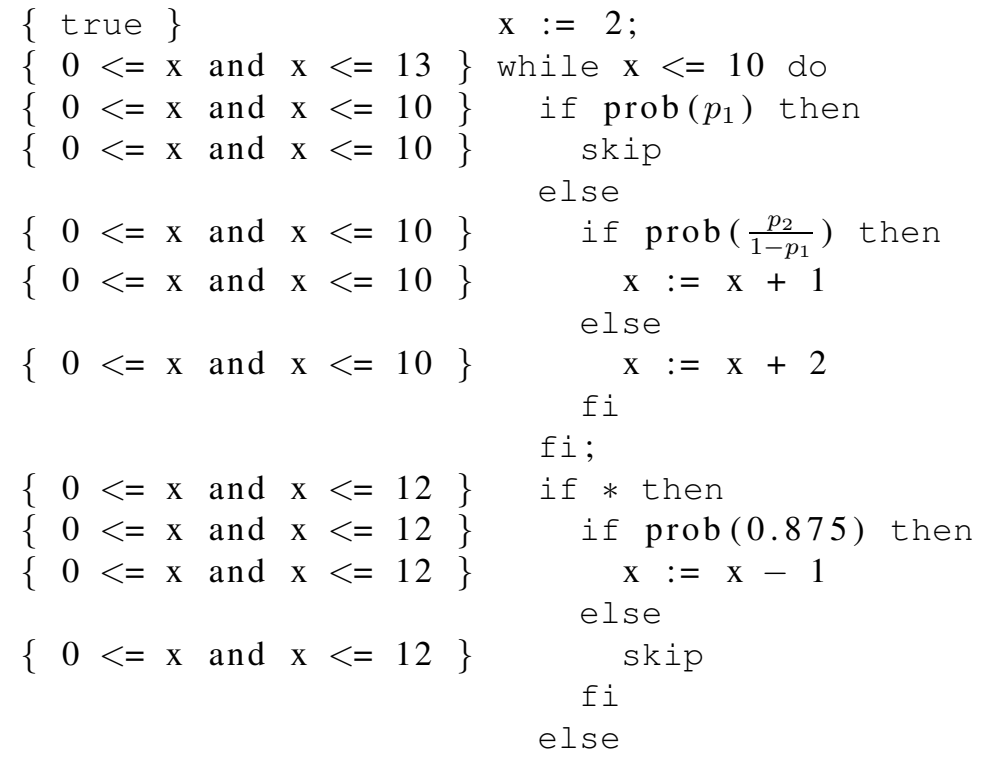




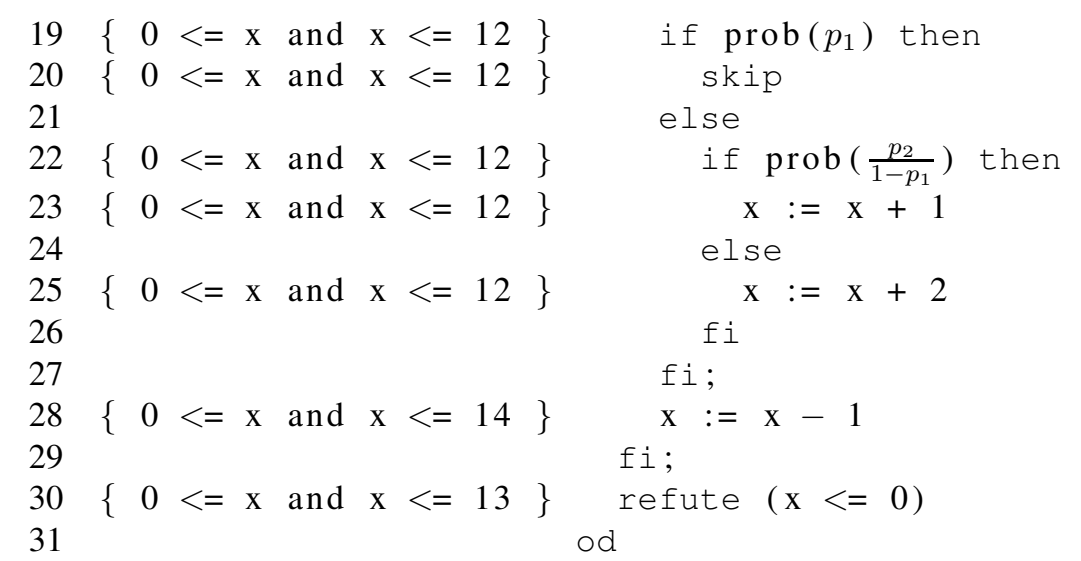

Here $p_{1}, p_{2} \in[0,1]$.

(a-2) (2D adversarial random walk [12]) It is a random walk over $\mathbb{R}^{2}$ with a time limit of 100 rounds. The process starts from $(x, y)=(2,2)$ and in each round, either " $x$ " or " $y$ " is nondeterministically chosen, and it is added by $z \in \mathbb{R}$ that is uniformly chosen from $\left[M_{1}, M_{2}\right]$. The process terminates if $x \leq 0$ or $y \leq 0$.

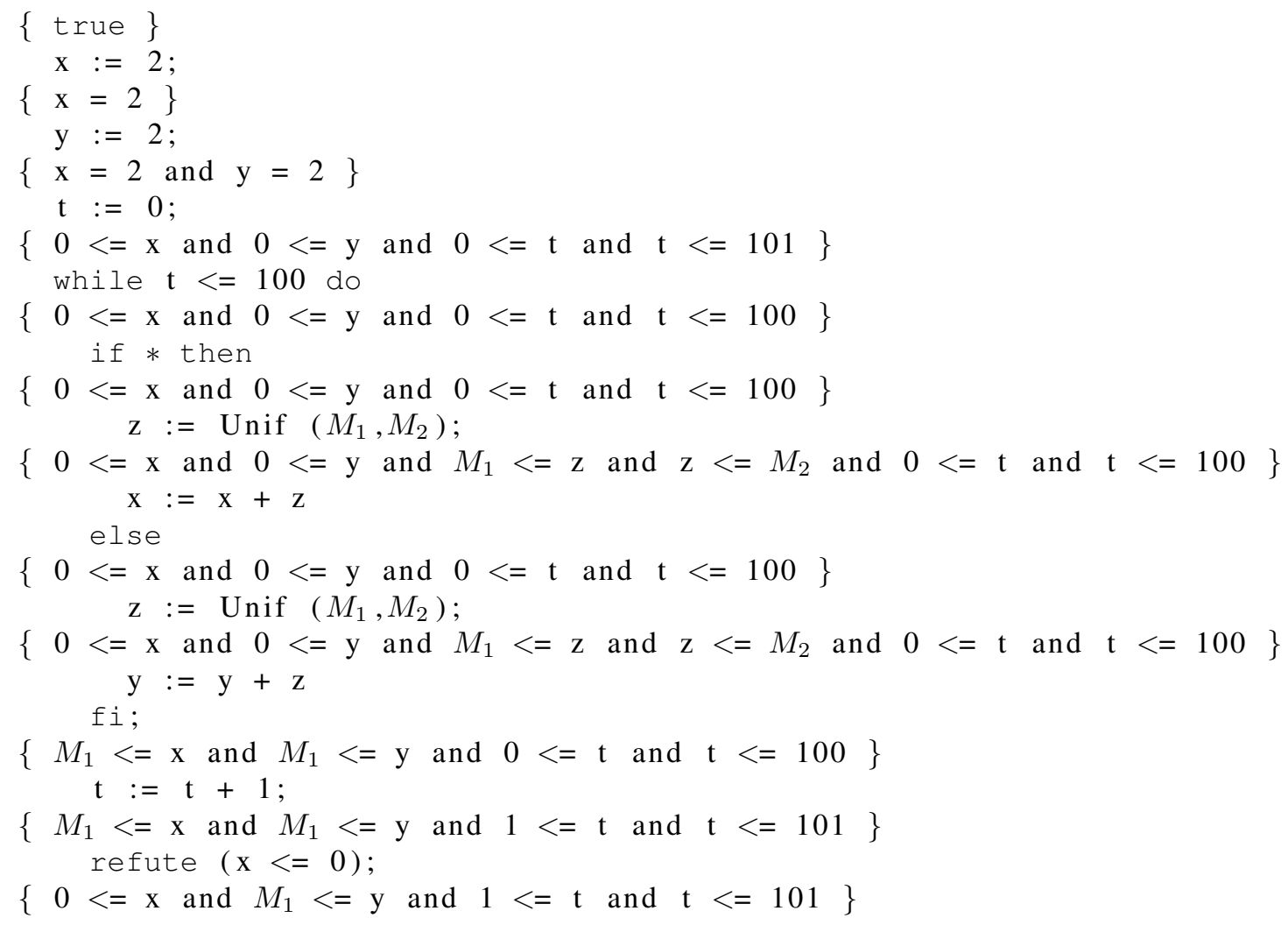




$$
\text { refute }(\mathrm{y}<=0)
$$

Here $M_{1}, M_{2} \in \mathbb{R}$.

(a-3) (variant of 2D adversarial random walk [12]) It is a random walk over $\mathbb{R}^{2}$ with a time limit of 100 rounds. The process starts from $(x, y)=(3,2)$, and in each round either of the following is nondeterministically chosen: (1) $z \in \mathbb{R}$ is uniformly chosen from $\left[M_{1}, M_{2}\right]$ and added to $x$ (resp. $y$ ) in probability 0.7 (resp. 0.3 ); or (2) $z \in \mathbb{R}$ is uniformly chosen from $\left[-M_{2},-M_{1}\right]$ and added to $y$ (resp. $x$ ) in probability 0.7 (resp. $0.3)$.

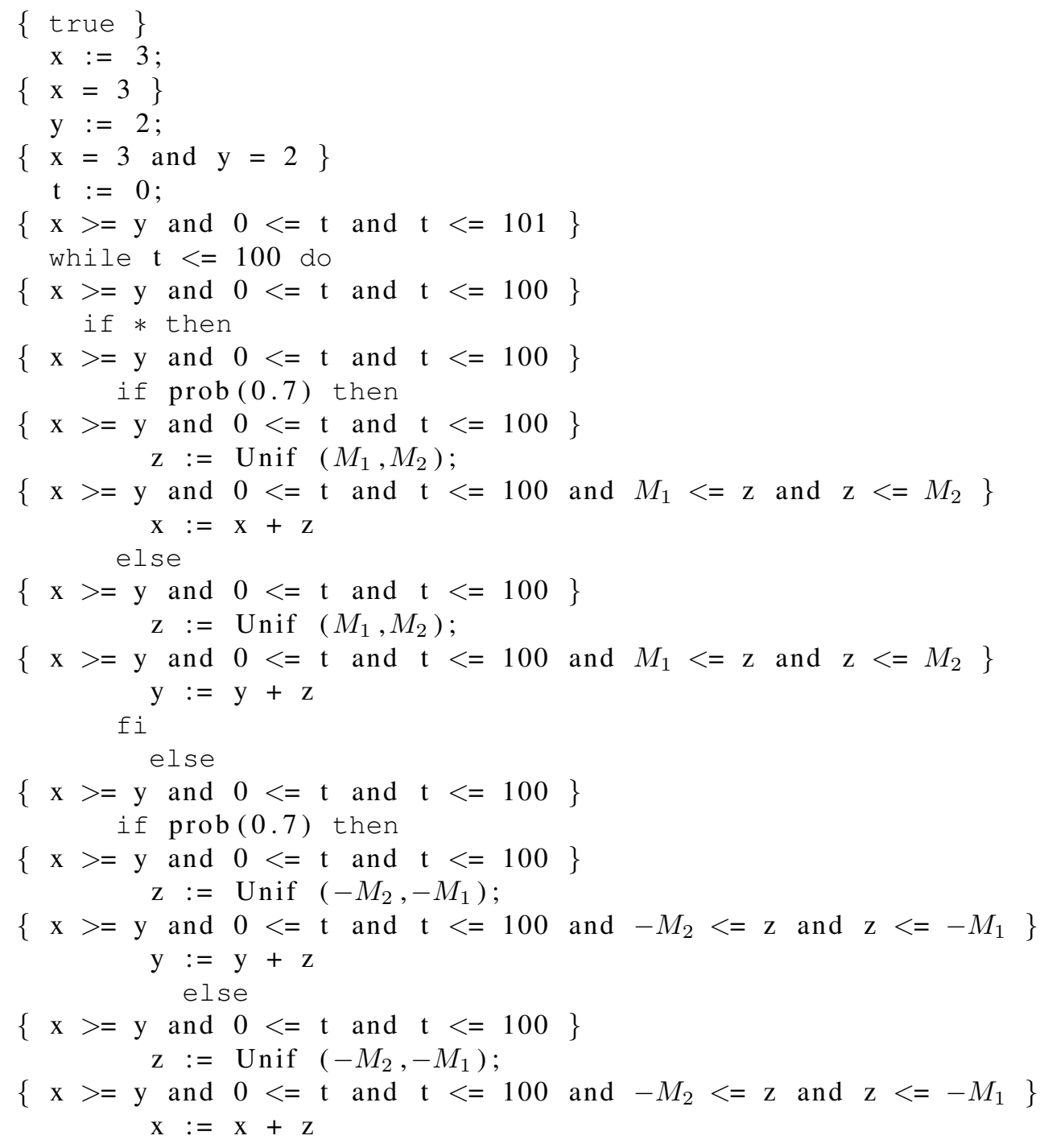




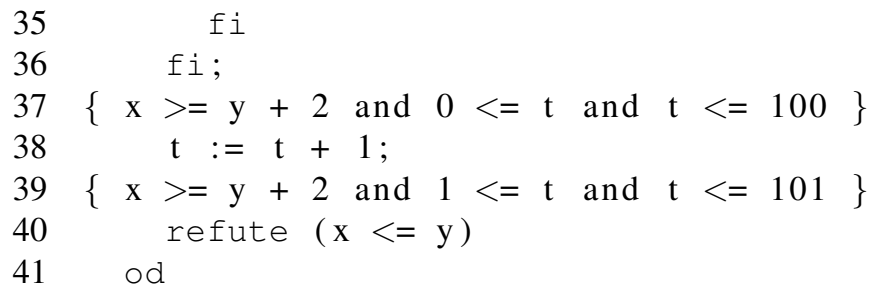

Here $M_{1}, M_{2} \in \mathbb{R}$.

(b) (room temperature control [19]) The rooms are named Room 1 and Room 2, and we write $x_{1}$ and $x_{2}$ for their temperature, with a time limit. The changes of $x_{1}$ and $x_{2}$ are modeled by the following stochastic difference equation: $x_{i}^{\prime}=x_{i}+a \cdot\left(x_{j}-\right.$ $\left.x_{i}\right)+b_{i} \cdot\left(x_{0}-x_{i}\right)+c_{i} \cdot \sigma\left(x_{i}\right)+\nu_{i}$ where $i, j \in\{1,2\}$ and $j \neq i$. The second term models the heat exchange between the rooms and the third term models the heat exchange with the outside whose temperature is $x_{0}$. The forth term models the influence of the air conditioner that is controlled by a function $\sigma: \mathbb{R} \rightarrow \mathbb{R}$. The last term $\nu_{i}$ is a probabilistic distribution that models the perturbation. Partially referring to [9], we have fixed the parameters as $a=0.0625, b_{1}=0.0375, b_{2}=0.025, c_{1}=c_{2}=c$, $\sigma(x)=19.5-x$ and $\nu_{1}, \nu_{2}$ independently follow the uniform distribution over $[-p, p]$, where $c, p \in \mathbb{R}$. The process terminates if $x_{1}$ or $x_{2}$ is out of the comfortable range: $[17,22]$ for $x_{1}$ and $[16,23]$ for $x_{2}$.

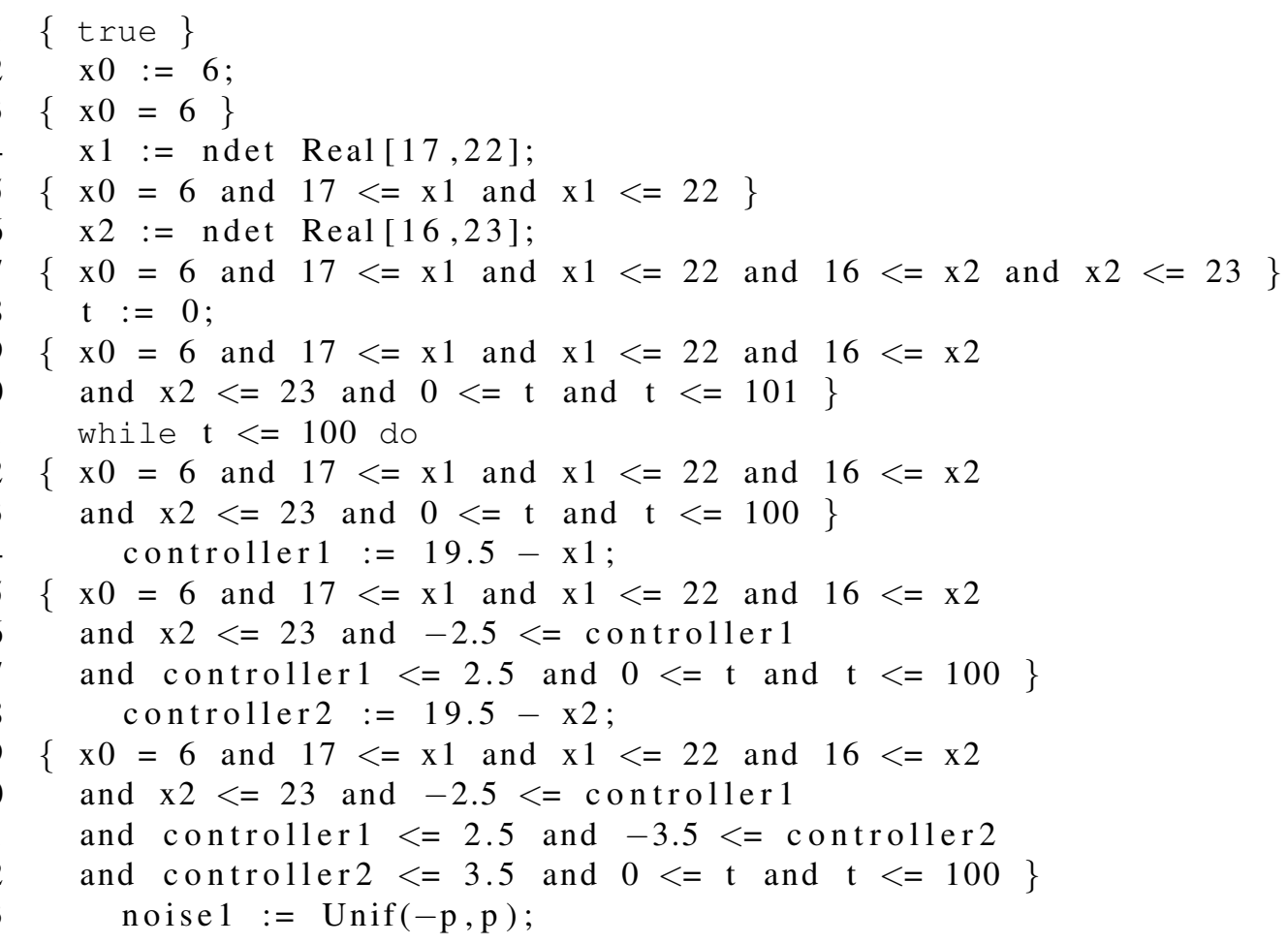




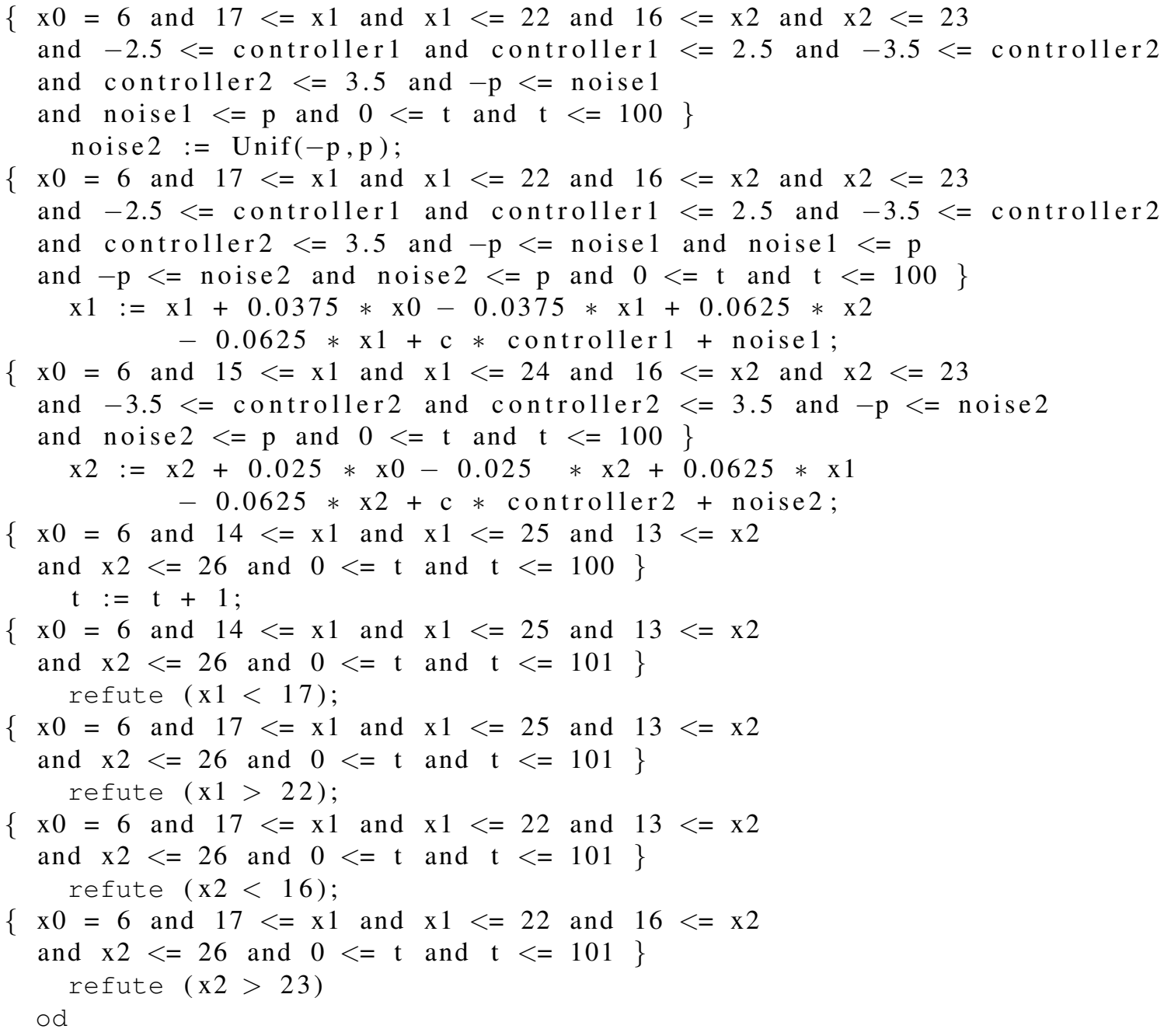

Here $c, p \in \mathbb{R}$.

\section{H pCFGs Used in Experiments}

The pCFGs $\mathcal{C}=\left(L, V, l_{\text {init }}, \boldsymbol{x}_{\text {init }}, \mapsto, \mathrm{Up}, \operatorname{Pr}, G\right)$ used to compare NNRepSupM and $\varepsilon$-RepSupM were as follows:

\section{(d-1) (bounded random walk over $[0,10]$ )}

- $L_{N}=\emptyset, L_{P}=\left\{l_{1}\right\}, L_{D}=\left\{l_{0}\right\}$ and $L_{A}=\left\{l_{2}, l_{3}, l_{4}\right\}$.

- $V=\{x\}$.

$-l_{\text {init }}=l_{0}$

$-\boldsymbol{x}_{\text {init }}=[x=5]$. 
- $\mapsto=\left\{\left(l_{0}, l_{1}\right),\left(l_{0}, l_{4}\right),\left(l_{1}, l_{2}\right),\left(l_{1}, l_{3}\right),\left(l_{2}, l_{0}\right),\left(l_{3}, l_{0}\right),\left(l_{4}, l_{4}\right)\right\}$.

- $\operatorname{Up}\left(l_{2}\right)=(x, \lambda x \cdot x-1), \operatorname{Up}\left(l_{3}\right)=(x, \lambda x \cdot x+1)$ and $\operatorname{Up}\left(l_{4}\right)=(x, \lambda x \cdot x+1)$.

- $\operatorname{Pr}\left(l_{1}, l_{2}\right)=0.1, \operatorname{Pr}\left(l_{1}, l_{3}\right)=0.9$.

- $G\left(l_{0}, l_{1}\right)=(x<10)$ and $G\left(l_{0}, l_{4}\right)=(x \geq 10)$.

Moreover, let $C:=\left\{\left(l_{1},[x \mapsto r]\right) \mid r \leq 0\right\} \subseteq L \times \mathbb{R}^{V}$, and let $\mathfrak{C}$ be a linear predicate map representing $C$. The pCFG represents a bounded random walk, and can be illustrated as follows (for simplicity, we are omitting some configurations).

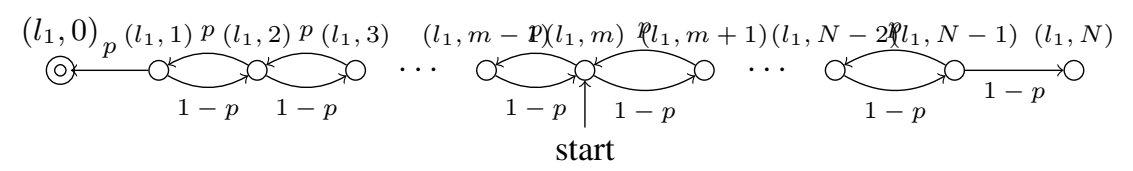

\section{(d-2) (simple system with infinite branching)}

- $L_{N}=\emptyset, L_{P}=\left\{l_{4}, l_{5}\right\}, L_{D}=\left\{l_{2}\right\}$ and $L_{A}=\left\{l_{1}, l_{3}, l_{6}\right\}$.

$-V=\{x\}$.

$-l_{\text {init }}=l_{1}$

- $\boldsymbol{x}_{\text {init }}=[x=0]$.

- $\mapsto=\left\{\left(l_{1}, l_{2}\right),\left(l_{2}, l_{3}\right),\left(l_{2}, l_{4}\right),\left(l_{3}, l_{2}\right),\left(l_{4}, l_{5}\right),\left(l_{4}, l_{6}\right),\left(l_{5}, l_{5}\right),\left(l_{6}, l_{6}\right)\right\}$.

- $\mathrm{Up}\left(l_{1}\right)=(x$, Geometric $(0.5))$ and $\operatorname{Up}\left(l_{3}\right)=\mathrm{Up}\left(l_{6}\right)=(x, \lambda x . x-1)$ where Geometric $(0.5)$ denotes a geometric distribution that takes a value $i \in \mathbb{N} \backslash\{0\}$ in a probability $0.5^{i}$.

- $\operatorname{Pr}\left(l_{4}, l_{5}\right)=\operatorname{Pr}\left(l_{4}, l_{6}\right)=0.5, \operatorname{Pr}\left(l_{5}, l_{5}\right)=1$.

- $G\left(l_{2}, l_{3}\right)=(x \geq 1)$ and $G\left(l_{2}, l_{4}\right)=(x<1)$.

Moreover, let $C=\left\{\left(l_{5},[x \mapsto r]\right) \mid r \in \mathbb{R}\right\} \subseteq L \times \mathbb{R}^{V}$, and assume that $\mathfrak{C}$ is a linear predicate map representing $C$. It can be illustrated as follows (for simplicity, we are omitting or merging some configurations).

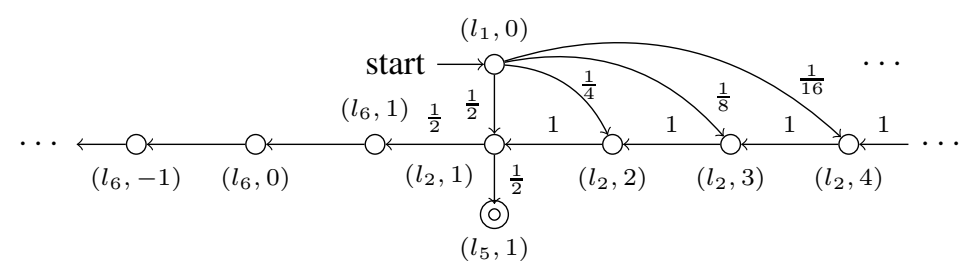

Remark H.1. In fact, there exists no RepSupM that can refute almost-sure termination. Let $\eta: L \times \mathbb{R}^{V} \rightarrow \mathbb{R}$ be an $\varepsilon$-RepSupM for $C$ that has $\kappa$-bounded differences for some $\kappa>0$. Assume that $f\left(l_{1}, 0\right)=A<0$. By Def. 3.1, we have:

$$
\eta\left(l_{2}, 1\right) \leq \eta\left(l_{3}, 2\right)-\varepsilon \leq \eta\left(l_{2}, 2\right)-2 \varepsilon \leq \eta\left(l_{3}, 3\right)-3 \varepsilon \leq \eta\left(l_{2}, 3\right)-4 \varepsilon \leq \cdots .
$$

Hence $\eta\left(l_{2}, n\right) \geq \eta\left(l_{2}, 1\right)+2(n-1) \varepsilon$, and therefore $\lim _{n \rightarrow \infty} \eta\left(l_{2}, n\right)=\infty$. This contradicts to the $\kappa$-bounded condition.

In contrast, if we define a linear expression map $\mathfrak{f}$ and a linear predicate map $\mathfrak{I}$ by $\mathfrak{f}\left(l_{1}\right)=\mathfrak{f}\left(l_{2}\right)=\mathfrak{f}\left(l_{3}\right)=\mathfrak{f}\left(l_{4}\right)=\frac{1}{2}, \mathfrak{f}\left(l_{5}\right)=1, \mathfrak{f}\left(l_{6}\right)=0$ and $\mathfrak{I}(l)=$ true for each $l \in L$, then $\mathfrak{f}$ is a linear $\mathrm{U}-\mathrm{NNRepSupM}$ at $\mathfrak{C}$ and 1 supported by $\mathfrak{I}$. 


\section{(d-3) (random walk over $[0,1]$ that exhibits geometric behaviors)}

- $L_{N}=\emptyset, L_{P}=\left\{l_{3}\right\}, L_{D}=\left\{l_{2}\right\}$ and $L_{A}=\left\{l_{1}, l_{4}, l_{5}, l_{6}\right\}$.

- $V=\{x\}$.

$-l_{\text {init }}=l_{1}$

- $\boldsymbol{x}_{\text {init }}=[x=0]$.

- $\mapsto=\left\{\left(l_{1}, l_{2}\right),\left(l_{2}, l_{3}\right),\left(l_{2}, l_{6}\right),\left(l_{3}, l_{4}\right),\left(l_{3}, l_{5}\right),\left(l_{4}, l_{2}\right),\left(l_{5}, l_{2}\right),\left(l_{6}, l_{6}\right)\right\}$.

- $\operatorname{Up}\left(l_{1}\right)=(x, \operatorname{Uniform}[0,1]), \operatorname{Up}\left(l_{4}\right)=(x, \lambda x .2 * x), \operatorname{Up}\left(l_{5}\right)=(x, \lambda x .0 .5 *$ $x)$ and $\operatorname{Up}\left(l_{6}\right)=(x, \lambda x . x)$ where Uniform $[0,1]$ is the uniform distribution over $[0,1]$.

- $\operatorname{Pr}\left(l_{3}, l_{4}\right)=0.25, \operatorname{Pr}\left(l_{3}, l_{5}\right)=0.75$.

- $G\left(l_{2}, l_{3}\right)=(x<1)$ and $G\left(l_{2}, l_{6}\right)=(x \geq 1)$.

Moreover, let $C=\left\{\left(l_{6},[x \mapsto r]\right) \mid r \in \mathbb{R}\right\} \subseteq L \times \mathbb{R}^{V}$, and let $\mathfrak{C}$ be a linear predicate map representing $C$.

Remark H.2. In fact, no $\varepsilon$-RepSupM can refute almost-sure reachability to $\llbracket \mathfrak{C} \rrbracket$. Let $\eta: L \times \mathbb{R}^{V} \rightarrow \mathbb{R}$ be an $\varepsilon$-RepSupM for $C$ that has $\kappa$-bounded differences for some $\kappa>0$. Assume $\eta\left(l_{1}, 0\right)=A<0$. Then we have: $A=\eta\left(l_{1}, 0\right) \geq \int_{x \in[0,1]} \eta\left(l_{2}, x\right)+\varepsilon$. Hence there exists $x>0$ such that $\eta\left(l_{2}, x\right) \leq A<0$.

Let $N \in \mathbb{N}$ be the minimum number such that $2^{N} x \geq 1$. As $\left(l_{6}, 2^{N} x\right) \in C$, we have $\eta\left(l_{2}, 2^{N} x\right) \geq \eta\left(l_{6}, 2^{N} x\right)+\varepsilon \geq 0$, which means that there exists $n^{\prime} \in\{0, \ldots, N-1\}$ such that $\eta\left(l_{2}, 2^{n^{\prime}} x\right)-\eta\left(l_{2}, 2^{n^{\prime}+1} x\right)>0$.

For each $n \in \mathbb{Z}$ such that $n \leq N-1$, we have:

$$
\begin{aligned}
\eta\left(l_{2}, 2^{n} x\right) & \geq \eta\left(l_{3}, 2^{n} x\right)+\varepsilon \\
& \geq 0.25 \cdot \eta\left(l_{4}, 2^{n+1} x\right)+0.75 \cdot \eta\left(l_{5}, 2^{n-1} x\right)+2 \varepsilon \\
& \geq 0.25 \cdot \eta\left(l_{2}, 2^{n+1} x\right)+0.75 \cdot \eta\left(l_{2}, 2^{n-1} x\right)+3 \varepsilon .
\end{aligned}
$$

This implies:

$$
\eta\left(l_{2}, 2^{n-1} x\right)-\eta\left(l_{2}, 2^{n} x\right) \leq \frac{0.25}{0.75}\left(\eta\left(l_{2}, 2^{n} x\right)-\eta\left(l_{2}, 2^{n+1} x\right)\right)-\frac{0.25}{0.75} \cdot 3 \varepsilon .
$$

By the two discussions above, we can inductively prove $\eta\left(l_{2}, 2^{n} x\right)-\eta\left(l_{2}, 2^{n+1} x\right) \leq$ $-\frac{1}{3} \cdot 3\left(n-n^{\prime}\right) \varepsilon$ for each $n \leq n^{\prime}$. Hence $\lim _{n \rightarrow \infty} f\left(l_{2}, 2^{n} x\right)-f\left(l_{1}, 0\right)=-\infty$, and it contradicts to the $\kappa$-bounded condition.

In contrast, if we define a linear expression map $\mathfrak{f}$ and a linear predicate map $\mathfrak{I}$ by $\mathfrak{f}\left(l_{1}\right)=\frac{1}{2}, \mathfrak{f}\left(l_{2}\right)=\mathfrak{f}\left(l_{3}\right)=x, \mathfrak{f}\left(l_{4}\right)=2 x, \mathfrak{f}\left(l_{5}\right)=\frac{1}{2} x$ and $\mathfrak{I}(l)=(x \geq 0)$ for each $l \in L$, then $\mathfrak{f}$ is a linear NNRepSupM for $\mathfrak{C}$ at 1 supported by $\mathfrak{I}$.

\section{(d-4) (unbounded random walk)}

- $L_{N}=\emptyset, L_{P}=\left\{l_{1}\right\}, L_{D}=\emptyset$ and $L_{A}=\left\{l_{2}, l_{3}\right\}$.

$-V=\{x\}$.

$-l_{\text {init }}=l_{1}$

$-\boldsymbol{x}_{\text {init }}=[x=1]$. 
- $\mapsto=\left\{\left(l_{1}, l_{2}\right),\left(l_{1}, l_{3}\right),\left(l_{2}, l_{1}\right),\left(l_{3}, l_{1}\right)\right\}$.

- $\mathrm{Up}\left(l_{2}\right)=(x, \lambda x . x-1)$ and $\mathrm{Up}\left(l_{3}\right)=(x, \lambda x \cdot x+1)$.

- $\operatorname{Pr}\left(l_{1}, l_{2}\right)=0.25, \operatorname{Pr}\left(l_{1}, l_{2}\right)=0.75$.

- $G$ is the empty function.

We define $C \subseteq L \times \mathbb{R}^{V}$ by $C:=\left\{\left(l_{1},[x \mapsto r]\right) \mid r \leq 0\right\}$, and let $\mathfrak{C}$ be a linear predicate map representing $C$. 